\title{
BLIND RELAY NETWORK WITH VITERBI DETECTION
}

\author{
A Thesis by \\ Shuang Feng \\ Bachelor of Science, Wichita State University, 2008
}

Submitted to the Department of Electrical Engineering and the faculty of the Graduate School of

Wichita State University

in partial fulfillment of

the requirements for the degree of Master of Science 
(C) Copyright 2010 by Shuang Feng All Rights Reserved 


\section{ANALYSIS OF DIFFERENT DETECTION SCHEMES IN RELAY CASE}

The following faculty members have examined the final copy of this thesis for form and content, and recommend that it be accepted in partial fulfillment of the requirement for the degree of Master of Science with a major in Electrical Engineering.

Hyuck Kwon, Committee Chair

$\overline{\text { Yanwu Ding, Committee Member }}$

Xiaomi Hu, Committee Member 


\section{ACKNOWLEDGMENTS}

I would like to thank my adviser, Dr. Hyuck Kwon, for his guidance and support. I also thank Dr. Yanwu Ding and Dr. Xiaomi Hu for their assistance and patience. Finally, I would many thanks to all of my friends who helped and cared about me. 


\section{ABSTRACT}

This thesis compared the performance of a blind relay communication system with that of a non-blind relay communication system. For a blind system, a differential space-time block code (DSTBC) or a blind Viterbi detection were used, and for a nonblind system, a space-time block code (STBC) was used. Also, this thesis took another blind detection mechanism, the Zhang's code, and compared its performance with that of the blind Viterbi detection under a Jakes' fading environment. It was found that the case of a relay closer to the destination shows better performance than the other case of a relay closer to the destination. It was also observed that the blind Viterbi decoder shows better performance than the Zhang's code under the Jakes' fading environment. 


\section{TABLE OF CONTENTS}

Chapter

Page

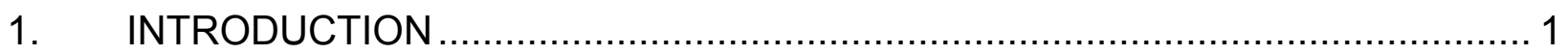

$1.1 \quad$ Introduction to Relay System ........................................................... 1

1.2 Introduction to Coherent and Noncoherent Detection .......................... 1

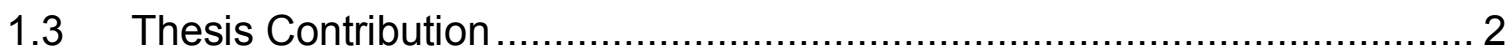

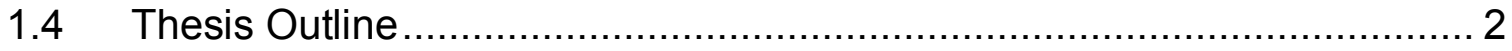

2. REVIEW OF COHERENT AND NONCOHERENT SCHEMES ......................... 3

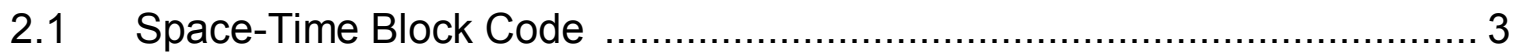

2.2 Differential Space-Time Block Code ............................................ 4

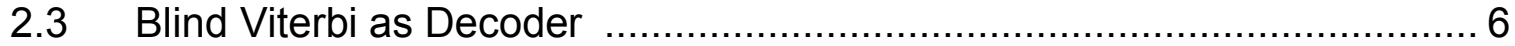

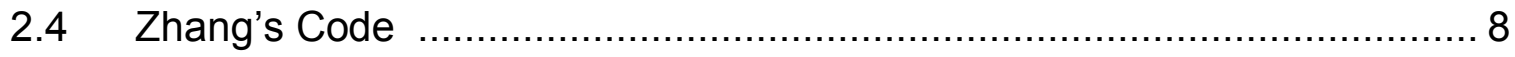

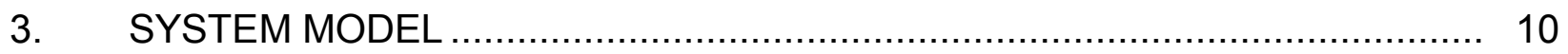

3.1 Detection Schemes Combined with Decode and Forward Protocol ........ 10

3.1.1 Combined with STBC ….................................................... 10

3.1.2 Combined with DSTBC and Blind Viterbi Decoder .................. 12

3.1.3 Parallel-Relay Case ........................................................... 12

3.2 Detection Schemes Combined with Amplify and Forward Protocol .......... 13

3.2.1 Combined with STBC ….................................................... 13

3.2.2 Combined with DSTBC and Blind Viterbi Decoder .................. 15

3.3 Zhang's Code and Blind Viterbi Decoder under Jakes' Fading ............... 15

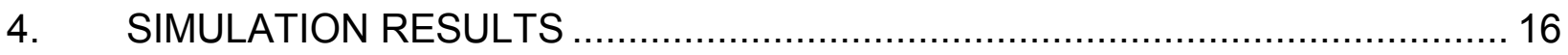

4.1 Simulation Parameters................................................................ 16

4.2 DF Relay Combined with STBC .................................................... 16

4.3 DF Relay Combined with DSTBC ….......................................... 17

4.4 DF Relay Combined with Blind Viterbi Decoder .................................. 18

4.5 Single AF Relay ................................................................... 19

4.6 SER Performance of Zhang's Code and Blind Viterbi........................... 20

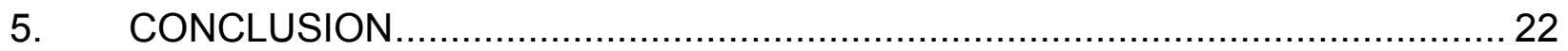

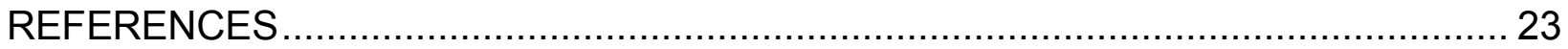


TABLE OF CONTENTS (Cont.)

Chapter

Page

APPENDIXES

A. Program of DF Relay Combined with STBC …................................... 26

B. Program of DF Relay Combined with DSTBC .................................. 28

C. Program of DF Relay Combined with Blind Viterbi Decoder .................... 31

D. Program of AF Relay Combined with DSTBC.................................. 37

E. Program of DSTBC Relay Combined with AF................................... 43

F. Program of Zhang's Code in Case of Jakes' Fading .............................. 45

G. Program of QPSK Blind Viterbi in case of Jakes' Fading ........................ 49 


\section{LIST OF TABLES}

Table

2.1 STBC Encoding and Transmission Sequence .................................................... 3

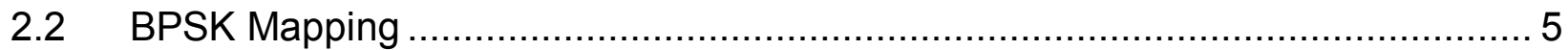

2.3 Coefficient Vector Set V for BPSK ......................................................... 7

$2.4 \quad$ Coefficient Vector Set V for QPSK ......................................................... 7

3.1 Procedure of STBC Combined with DF Relay …....................................... 12

3.2 Procedure of STBC Combined with AF Relay .......................................... 14 


\section{LIST OF FIGURES}

Figure

Page

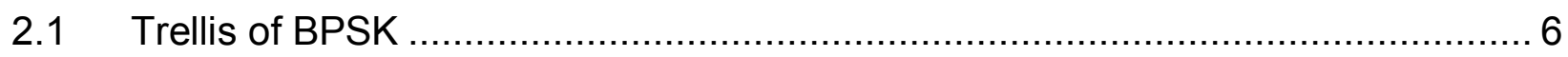

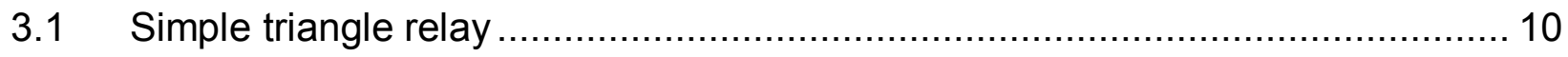

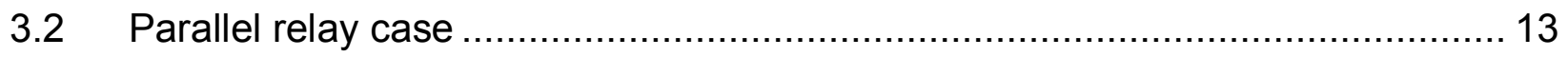

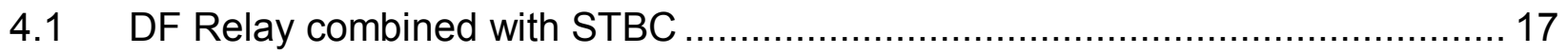

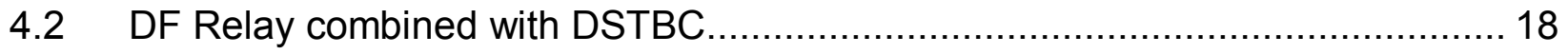

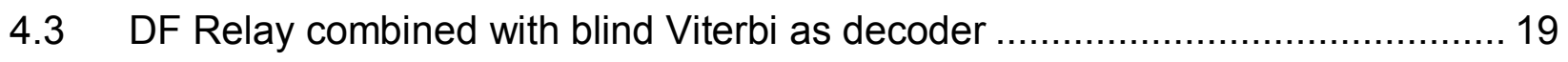

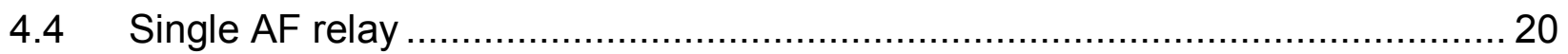

4.5 BER performance of Zhang's code and blind Viterbi as decoder under

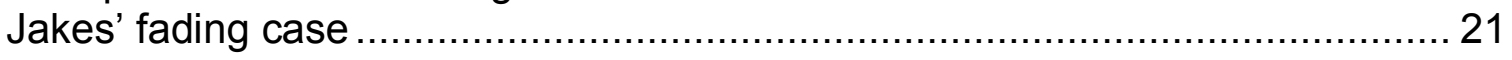




\section{LIST OF ABBREVIATIONS/NOMENCLATURE}

$\begin{array}{ll}\text { AF } & \text { Amplify and Forward } \\ \text { BER } & \text { Bit Error Rate } \\ \text { BPSK } & \text { Binary Phase Shift Keying } \\ \text { dB } & \text { Decibel } \\ \text { DF } & \text { Decode and Forward } \\ \text { Dsd } & \text { Distance between Source and Relay } \\ \text { DSTBC } & \text { Differential Space-Time Block Code } \\ \text { QPSK } & \text { Quadrature Phase Shift Keying } \\ \text { SER } & \text { Symbol Error Rate } \\ \text { SNR } & \text { Signal-to-Noise Ratio } \\ \text { STBC } & \text { Space-Time Block Code } \\ \text { 3PSK } & \text { Three Phase Shift Keying }\end{array}$




\section{CHAPTER 1}

\section{INTRODUCTION}

\subsection{Introduction to Relay System}

A typical amplify-and-forward (AF) relay network consists of a source, a relay, and a destination. A source transmits its message to both the relay and the destination during the first slot time interval. And the relay amplifies its received signal and transmits to the destination during the second slot time interval. The destination combines the signals received from both the source and the relay during the first and second slot time intervals, respectively.

A typical decode-and-forward (DF) relay network decodes the information at the relay, and re-encodes and transmits it to the destination during the second slot time interval. There are many variations [1].

\subsection{Introduction to Coherent and Noncoherent Detection}

Coherent detection means the channel coefficients are known to the relay and the destination. For example, the space-time block code (STBC) requires the channel coefficient information for detection. On the other hand, noncoherent detection means that the channel coefficients are unknown to the relay and the destination. For example, the following do not require channel coefficient information: differential space-time block code (DSTBC) as used by Tarokh and Jafarkhani [2], the blind Viterbi detection as used by Shao et al. [3], and blind detection as used by Zhang and Ma [4]. 


\subsection{Thesis Contribution}

The contribution of this thesis is the application of various instances of blind detection [2], [3], [4] in a relay network and comparisons of their performances.

\subsection{Thesis Outline}

This thesis is organized as follows:

- Chapter 2. Review of Coherent and Noncoherent Schemes

- Chapter 3. System Model

- Chapter 4. Simulation Results

- Chapter 5. Conclusion 


\section{CHAPTER 2}

\section{REVIEW OF COHERENT AND NONCOHERENT SCHEMES}

\subsection{Space-Time Block Code}

In this thesis, a system with two transmit antennas and one receive antenna was considered. The modulation set was given as

$$
A=\left\{\frac{e^{\frac{2 \pi k j}{M}}}{\sqrt{2}}, k=0,1, \ldots, M-1\right\}
$$

where $M=2^{b}, \sqrt{-1}=j, b=$ the number of bits per symbol, and the division with $\sqrt{2}$ is for the normalized power shared by two transmit antennas.

At the first symbol time, two symbols will be sent simultaneously by two antennas. The symbol sent by antenna one is denoted by $s_{1}$, and the symbol sent by antenna two is denoted by $s_{2}$. At the next symbol time, $-s_{2}^{*}$ will be sent by antenna one, and $s_{1}^{*}$ will be sent by antenna two, where the superscript $\left({ }^{*}\right)$ is the complex conjugate operation. Therefore, the STBC can be represented as shown in Table 2.1

TABLE 2.1

THE STBC ENCODING AND TRANSMISSON SEQUENCE

\begin{tabular}{|l|c|c|}
\hline & Antenna One & Antenna Two \\
\hline First Symbol Time & $s_{1}$ & $s_{2}$ \\
\hline Second Symbol Time & $-s_{2}^{*}$ & $s_{1}^{*}$ \\
\hline
\end{tabular}

At the receiver, the received signals in those two symbol periods, $r_{1}$ and $r_{2}$, can be expressed as 


$$
\begin{aligned}
& \mathrm{r}_{1}=\mathrm{h}_{1} \mathrm{~s}_{1}+\mathrm{h}_{2} \mathrm{~s}_{2}+\mathrm{n}_{1} \\
& \mathrm{r}_{2}=-\mathrm{h}_{1} \mathrm{~s}_{2}^{*}+\mathrm{h}_{2} \mathrm{~s}_{1}^{*}+\mathrm{n}_{2}
\end{aligned}
$$

where $r_{t}, t=1,2$ indicates the received signals at the $t$-th symbol time; $h_{i}, i=1,2$ are the fading coefficients for the $\mathrm{i}$-th transmitter to the receiver; and $n_{i}, i=1,2$ are the complex Guassian noise random variables.

For the STBC, it was assumed that the receivers completely know the channel state information. Therefore, the STBC is a coherent detection scheme. Then the $S_{1}$ and $S_{2}$ can be estimated as

$$
\begin{gathered}
\hat{s}_{1}=\mathrm{h}_{1}^{*} \mathrm{r}_{1}+h_{2} r_{2}^{*} \\
\hat{s}_{2}=\mathrm{h}_{2}^{*} \mathrm{r}_{1}-h_{1} r_{2}^{*} .
\end{gathered}
$$

Those combined signals, $\hat{s}_{1}$ and $\hat{s}_{2}$, will be decoded by the maximum likelihood detector. Having $s_{1}$ and $s_{2}$ estimated, this process is repeated to estimate $s_{3}, s_{4}, \ldots$ until the end-of-information symbols are in a frame.

\subsection{Differential Space-Time Block Code}

The constellation was restricted to be the same as the STBC case, as shown in equation (2.1). The STBC uses two symbols (one block) to decode two symbols (one block), Here, the DSTBC uses four symbols (two blocks) to decode two symbols (one block) [2]. Therefore, there should be four received combining signals from the first symbol time to the fourth symbol time as follows:

$$
\begin{aligned}
& \mathrm{r}_{1}=\mathrm{h}_{1} \mathrm{~s}_{1}+\mathrm{h}_{2} \mathrm{~s}_{2}+\mathrm{n}_{1} \\
& \mathrm{r}_{2}=-\mathrm{h}_{1} \mathrm{~s}_{2}^{*}+\mathrm{h}_{2} \mathrm{~s}_{1}^{*}+\mathrm{n}_{2} \\
& \mathrm{r}_{3}=\mathrm{h}_{1} \mathrm{~s}_{3}+\mathrm{h}_{2} \mathrm{~s}_{4}+\mathrm{n}_{3} \\
& \mathrm{r}_{4}=-\mathrm{h}_{1} \mathrm{~s}_{4}^{*}+\mathrm{h}_{2} \mathrm{~s}_{3}^{*}+\mathrm{n}_{4} .
\end{aligned}
$$


Because the complex vectors $\left(s_{1} s_{2}\right)$ and $\left(-s_{2}^{*}, s_{1}^{*}\right)$ are orthogonal to each other and have unit lengths, another two-dimensional vector at time $t,\left(s_{t} s_{t+1}\right)$ can be uniquely represented in the orthonormal basis given by these vectors. The orthonomal basis is denoted as

$$
\mathrm{D}_{1}=\left[\begin{array}{cc}
\mathrm{s}_{1} & \mathrm{~s}_{2} \\
-\mathrm{s}_{2}^{*} & \mathrm{~s}_{1}^{*}
\end{array}\right]
$$

where $D_{B}$ is the B-th block orthonomal basis. Then by using $D_{1}$, the $\left(s_{3}, s_{4}\right)$ can be uniquely represented as

$$
\left(\mathrm{s}_{3}, \mathrm{~s}_{4}\right)=\mathcal{R}_{1} \mathrm{D}_{1}=\mathcal{R}_{1}^{1}\left(\mathrm{~s}_{1}, \mathrm{~s}_{2}\right)+\mathcal{R}_{1}^{2}\left(-\mathrm{s}_{2}^{*}, \mathrm{~s}_{1}^{*}\right)
$$

where $\mathcal{R}_{1}=\left(\mathcal{R}_{1}^{1}, \mathcal{R}_{1}^{2}\right)$ is the coefficient vector.

At the decoder, without knowing the channel coefficients, the estimation of $\mathcal{R}_{B}$ is calculated from the received signals $r_{1}, r_{2}, r_{3}$, and $r_{4}$ as

$$
\begin{aligned}
& \tilde{\mathcal{R}}_{\mathrm{B}}^{1}=\mathrm{r}_{2 \mathrm{t}+1} \mathrm{r}_{2 \mathrm{t}-1}^{*}+\mathrm{r}_{2 \mathrm{t}+2}^{*} \mathrm{r}_{2 \mathrm{t}} \\
& \tilde{\mathcal{R}}_{\mathrm{B}}^{2}=\mathrm{r}_{2 \mathrm{t}+1} \mathrm{r}_{2 \mathrm{t}}^{*}-\mathrm{r}_{2 \mathrm{t}+2}^{*} \mathrm{r}_{2 \mathrm{t}-1}
\end{aligned}
$$

where $B$ indicates the $B$-th block of the vector $\mathcal{R}$.

The set $\mathrm{V}$ is defined as all possible vectors of $\mathcal{R}_{B}$. After $\mathcal{R}_{B}$ has been estimated, the receiver now computes the closest vector $\mathrm{V}$ to $\left(\widetilde{\mathcal{R}_{B}^{1}}, \widetilde{\mathcal{R}_{B}^{2}}\right)$, Once the vector is calculated, the inverse mapping of $\mathrm{V}$ will be made to decode original bits. For the binary phase shift keying (BPSK) case, the mapping is shown in Table 2.2

\section{TABLE 2.2}

\section{BPSK MAPPING}

\begin{tabular}{|c|c|}
\hline$\left(\mathrm{b}_{1}, \mathrm{~b}_{2}\right)$ & $\left(\mathcal{R}^{1}, \mathcal{R}^{2}\right)$ \\
\hline$(0,0)$ & $(1,0)$ \\
\hline$(1,0)$ & $(0,1)$ \\
\hline$(0,1)$ & $(0,-1)$ \\
\hline$(1,1)$ & $(-1,0)$ \\
\hline
\end{tabular}


where $b_{1}, b_{2}$ indicate the first and second transmitted bits, respectively.

Compared with the coherent STBC detection scheme, typically, a 3 decibel (dB) loss in signal-to-noise (SNR) ratio is expected for the non-blind DSTBC detection.

\subsection{Blind Viterbi as Decoder}

The same encoder of DSTBC is used for the blind Viterbi detection [3]. Hence, two blocks of signals that are the same as in equation (2.4) are received. By using equation (2.7), the receiver will calculate $\tilde{\mathcal{R}}_{\mathrm{B}}=\left(\tilde{\mathcal{R}}_{\mathrm{B}}^{1}, \tilde{\mathcal{R}}_{\mathrm{B}}^{2}\right)$, and then those vectors will be fed to the blind Viterbi decoder. The state of Viterbi trellis, $S$, indicates the original transmitted symbols. The pair of states $S_{j}^{t}$ and $S_{i}^{t-1}$ is linked by the trellis branch labeled by the coefficient vector $\mathcal{R}_{i j}$, where $\mathrm{i}$ and $\mathrm{j}$ indicate from the $\mathrm{i}$-th state to the $\mathrm{j}$-th state. The number of states is decided by modulation. In M-PSK, $i, j=1,2, \ldots, 2^{2 b}-1$. This assumes 200 symbols per frame, where the fading coefficients will not be changed.

For example, in the case of BPSK, the trellis is shown in Figure 2.1.

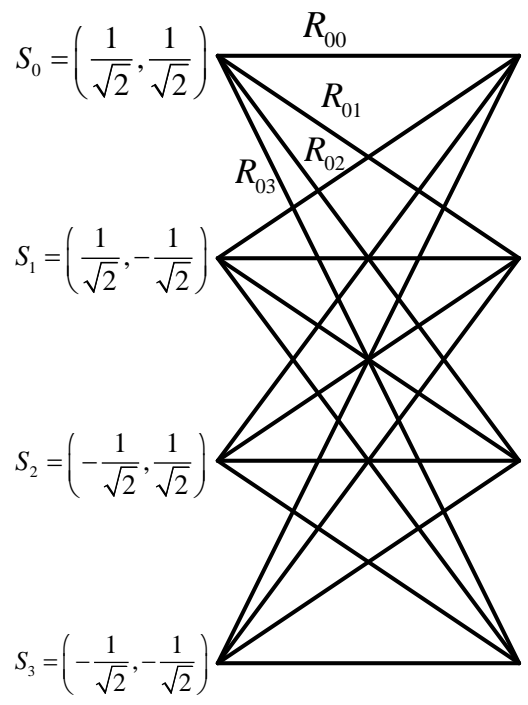

Figure 2.1. Trellis of BPSK. 
The size of $V$ is different if different modulation is used. For the BPSK case, the vectors of $\mathrm{V}$ are listed in Table 2.3

TABLE 2.3

COEFFICIENT VECTOR SET V FOR BPSK

\begin{tabular}{|l|l|}
\hline $\mathcal{R}_{00}=\mathcal{R}_{11}=\mathcal{R}_{22}=\mathcal{R}_{33}$ & $(1,0)$ \\
\hline $\mathcal{R}_{01}=\mathcal{R}_{13}=\mathcal{R}_{20}=\mathcal{R}_{32}$ & $(0,-1)$ \\
\hline $\mathcal{R}_{02}=\mathcal{R}_{10}=\mathcal{R}_{23}=\mathcal{R}_{31}$ & $(0,1)$ \\
\hline $\mathcal{R}_{03}=\mathcal{R}_{12}=\mathcal{R}_{21}=\mathcal{R}_{30}$ & $(-1,0)$ \\
\hline
\end{tabular}

All possible vectors of $\mathrm{V}$ for the quadrature phase shift keying (QPSK) modulation are listed in Table 2.4

TABLE 2.4

COEFFICIENT VECTOR SET V FOR QPSK

\begin{tabular}{|c|c|c|}
\hline$(1,0)$ & $(0.5+0.5 \mathrm{j},-0.5+0.5 \mathrm{j})$ & $(-0.5+0.5 \mathrm{j}, 0.5+0.5 \mathrm{j})$ \\
\hline$(-1,0)$ & $(0.5-0.5 \mathrm{j},-0.5-0.5 \mathrm{j})$ & $(-0.5+0.5 \mathrm{j},-0.5+0.5 \mathrm{j})$ \\
\hline$(0,1)$ & $(0.5+0.5 \mathrm{j}, 0.5-0.5 \mathrm{j})$ & $(0.5+0.5 \mathrm{j},-0.5+0.5 \mathrm{j})$ \\
\hline$(0,-1)$ & $(-0.5+0.5 \mathrm{j},-0.5-0.5 \mathrm{j})$ & $(0.5+0.5 \mathrm{j}, 0.5+0.5 \mathrm{j})$ \\
\hline$(\mathrm{j}, 0)$ & $(-0.5+0.5 \mathrm{j}, 0.5+0.5 \mathrm{j})$ & $(-0.5-0.5 \mathrm{j},-0.5+0.5 \mathrm{j})$ \\
\hline$(-\mathrm{j}, 0)$ & $(-0.5-0.5 \mathrm{j}, 0.5-0.5 \mathrm{j})$ & $(-0.5-0.5 \mathrm{j}, 0.5+0.5 \mathrm{j})$ \\
\hline$(0, \mathrm{j})$ & $(0.5-0.5 \mathrm{j}, 0.5+0.5 \mathrm{j})$ & $(0.5-0.5 \mathrm{j},-0.5+0.5 \mathrm{j})$ \\
\hline$(0,-\mathrm{j})$ & $(-0.5-0.5 \mathrm{j},-0.5+0.5 \mathrm{j})$ & $(0.5-0.5 \mathrm{j}, 0.5+0.5 \mathrm{j})$ \\
\hline
\end{tabular}

It can be seen that the size of V in BPSK is the same as the input information block set. But the size of V in QPSK is larger than the input information block set. This is 
due to redundancy introduced by the encoder. It is expected that the blind Viterbi detection is $1 \sim 1.5 \mathrm{~dB}$ better than the original blind detection scheme, i.e., DSTBC, for QPSK or a higher modulation case.

$\tilde{\mathcal{R}}_{\mathrm{B}}=\left(\tilde{\mathcal{R}}_{\mathrm{B}}^{1}, \tilde{\mathcal{R}}_{\mathrm{B}}^{2}\right)$ is calculated using equation (2.7). This estimated coefficient vectors will be fed to the Viterbi decoder. The branch metric, $\boldsymbol{Y}_{\mathrm{i}, \mathrm{j}}$, between state $\mathrm{i}$ to $\mathrm{j}$ is calculated as

$$
\boldsymbol{Y}_{\mathrm{i}, \mathrm{j}}(\mathrm{t})=\left\|\tilde{\mathcal{R}}_{\mathrm{B}}-\mathcal{R}_{i, j}\right\|^{2}
$$

\subsection{Zhang's Code}

The encoder used by Zhang and Ma [4] the same as the DSTBC used by Tarokh and Jafarkhani [2]. The difference is that the first block uses a p-ary PSK constellation, and the second block uses a q-ary PSK constellation, and p, q are co-prime. Therefore, at the receiver antenna, the received signals during the four consecutive time slots of the first and second blocks, $\mathrm{Z}_{1}$ and $\mathrm{Z}_{2}$, respectively, can be written in matrix form as

$$
\begin{aligned}
& \left(\begin{array}{l}
z_{1}(1) \\
z_{1}(2)
\end{array}\right)=\left(\begin{array}{cc}
x_{1} & x_{2} \\
-x_{2}^{*} & x_{1}^{*}
\end{array}\right)\left(\begin{array}{l}
h_{1} \\
h_{2}
\end{array}\right)+\left(\begin{array}{l}
n_{1} \\
n_{2}
\end{array}\right) \\
& \left(\begin{array}{ll}
\mathrm{z}_{2}(1) \\
\mathrm{z}_{2}(2)
\end{array}\right)=\left(\begin{array}{cc}
\mathrm{y}_{1} & \mathrm{y}_{2} \\
-\mathrm{y}_{2}^{*} & \mathrm{y}_{1}^{*}
\end{array}\right)\left(\begin{array}{l}
\mathrm{h}_{1} \\
\mathrm{~h}_{2}
\end{array}\right)+\left(\begin{array}{l}
\mathrm{n}_{3} \\
\mathrm{n}_{4}
\end{array}\right)
\end{aligned}
$$

where $\mathrm{x}$ and $\mathrm{y}$ are p-PSK modulation and q-PSK modulation symbols, respectively. Let S denote the symbol block $\left(\begin{array}{cc}x_{1} & x_{2} \\ -x_{2}^{*} & x_{1}^{*}\end{array}\right)$ or $\left(\begin{array}{cc}y_{1} & y_{2} \\ -y_{2}^{*} & y_{1}^{*}\end{array}\right)$, for $L=1$ single block.

In a part of the decoder, the original symbol block $S$ is estimated by extending it to $\mathrm{L}$ blocks as

$$
S=\underset{S \in A}{\arg \min }\left\{S^{H} P S\right\}
$$


where $S=\left(s_{1}^{*}(1), s_{1}(2), s_{2}^{*}(1), s_{2}(2), s_{3}^{*}(1), s_{3}(2), \ldots, s_{L}^{*}(1), s_{L}(2)\right)^{\mathrm{T}}, \mathrm{L}$ is the number of blocks, $\mathrm{P}=\left(\|\bar{z}\|_{2}^{2}+1\right) \mathrm{I}_{2 \mathrm{~L}}-\bar{z}^{H} \bar{z}$, and

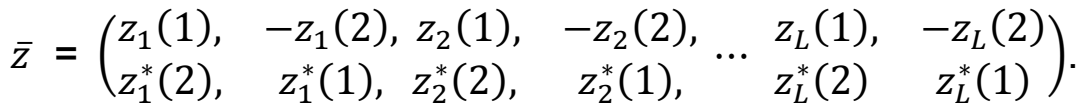

The simulation results from Zhang and Ma [4] show that the greater number of blocks for the Zhang's code shows better performance. In Chapter 4, it will be shown that if Zhang's code uses four blocks, then the performance is worse than the DSTBC. 


\section{CHAPTER 3}

\section{SYSTEM MODEL}

This thesis focused on a two-user cooperation scheme. In particular, the source (or user 1) sends information to the relay (or user 2) in timeslot 1, and the destination also receives the information. Then, the relay helps the source forward the information in timeslot 2, and the source also sends new information to its destination. This thesis considered a simple-triangle single-relay system, as shown in Figure 3.1 The channel coefficients are denoted by $h_{s r}, h_{r d}$, and $h_{s d}$ for the link from the source to the relay, the relay to the destination, and the source to the destination, respectively.

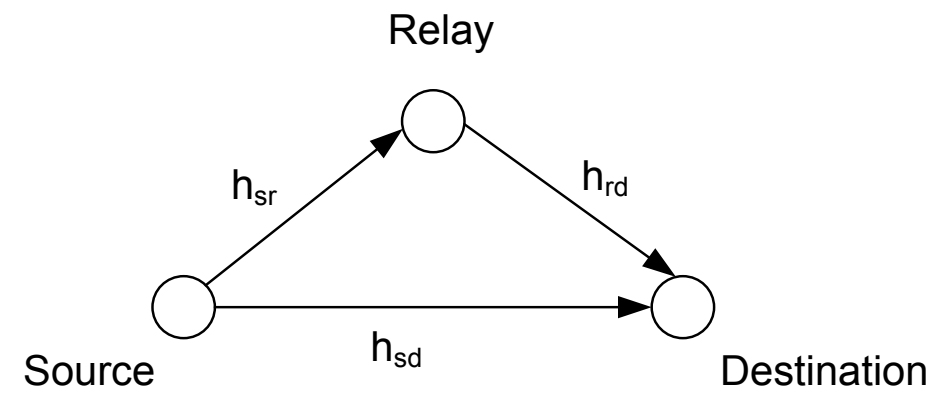

Figure 3.1. Simple triangle relay.

\subsection{Detection Schemes Combined with Decode and Forward Protocol}

\subsubsection{Combined with STBC}

For the STBC, to encode the first block symbol during two symbol intervals, it is necessary to feed the first block information to the encoder. The first symbol fed to encode is denoted as $S_{1}$, and the second is denoted as $S_{2}$. The received signal at the relay during the t-th symbol time is denoted by $Y_{s r t}$. Also, $Y_{s d t}$ denotes the received signal at the destination during the t-th symbol time, and $n_{s r t}$ and $n_{r d t}$ denote the zeromean AWGN noises corresponding to the received and destination links, respectively. 
When DF is used at the single relay, it takes four symbol times. At the first symbol time, the source sends $S_{1}$ to the relay; the relay receives the information and then decodes and re-encodes it. Hence, the received symbols at the relay during the first symbol time can be written as

$$
Y_{s r 1}=h_{s r} S_{1}+n_{s r 1} .
$$

After $S_{1}$ has been decoded and re-encoded, it will have the estimated symbol, $\tilde{S}_{1}$.

At the second symbol time, the relay forwards $\tilde{S}_{1}$ to the destination, and the source sends $S_{2}$ to its destination simultaneously. Hence, the received symbol at the destination during the second symbol time can be written as

$$
Y_{d 2}=h_{r d} \tilde{S}_{1}+h_{s d} S_{2}+n_{r d 2} .
$$

During the third symbol time, the source sends $\left(-S_{2}^{*}\right)$ to the relay, and the relay receives information and decodes and then re-encodes it. The received symbol during the tried symbol time at the relay can be written as

$$
Y_{s r 3}=h_{s r}\left(-S_{2}^{*}\right)+n_{s r 3} .
$$

Again, after $\left(-S_{2}^{*}\right)$ has been decoded and re-encoded, it will have the estimated symbol $-\tilde{S}_{2}^{*}$.

For the last symbol time, i.e., the fourth symbol time, the relay forwards $\tilde{S}_{2}^{*}$ to $\tilde{S}_{2}^{*}$ the destination, and the source sends $S_{1}^{*}$ to the destination simultaneously. The received symbols at the destination during the fourth symbol time can be written as

$$
Y_{d 4}=h_{s d} S_{1}^{*}+h_{r d}\left(-\tilde{S}_{2}^{*}\right)+n_{r d 2} .
$$

Hence, after four symbol times, a whole block will be sent by this relay system. It can be assumed that the fading coefficients do not change during the four symbol times. 
Chapter 4 will present bit error rate or symbol error rate performance of this scheme. As an example, this procedure is demonstrated in Table 3.1.

TABLE 3.1

PROCEDURE OF STBC COMBINED WITH DF RELAY

\begin{tabular}{|c|c|c|}
\hline$t=1$ & $s^{S_{1}}{ }^{{ }^{\circ} D}$ & $Y_{s r 1}=h_{s r} S_{1}+n_{s r 1}$ \\
\hline$t=2$ & $\mathrm{~S}_{\mathrm{S}_{2}}$ & $Y_{d 2}=h_{r d} \widetilde{S_{1}}+h_{s d} S_{2}+n_{r d 2}$ \\
\hline$t=3$ & $s^{0} S_{2}^{R}$ & $Y_{s r 3}=h_{s r}\left(-S_{2}^{*}\right)+n_{s r 3}$ \\
\hline $\mathrm{t}=4$ & $S^{\circ} S_{1}^{*}$ & $Y_{d 4}=h_{s d} S_{1}^{*}+h_{r d}\left(-\widetilde{S}_{2}^{*}\right)+n_{r d 2}$ \\
\hline
\end{tabular}

\subsubsection{Combined DSTBC and Blind Viterbi Decoder}

DSTBC needs two block symbols, which take four symbol times to decode one block. This can be done by repeating the STBC preprocessing but for eight symbol times. Also, blind Viterbi will work in the same way as the DSTBC because it also requires eight symbol times. Performance of those schemes will be shown in Chapter 4 .

\subsubsection{Parallel-Relay Case}

This section describes the location of reply considered in this thesis. The source, relay, and destination are parallel with each other. The distance between source and 
relay is $D_{s r}$, and the distance between the relay destination is $D_{r d}$. Figure 3.2 shows the single-relay case. Performance of this scheme will be presented in Chapter 4.

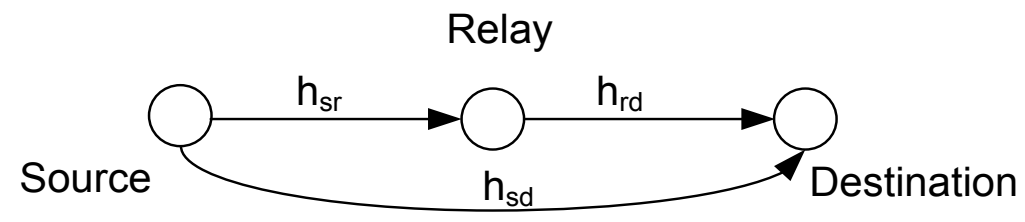

Figure 3.2. Parallel relay case.

\subsection{Detection Schemes Combined with Amplify-and-Forward Protocol}

\subsubsection{Combined with STBC}

The only difference compared with the DF protocol is that the AF relay will amplify the received information from the source and forward it to the destination without decoding and re-encoding as in the DF protocol.

Again, an entire procedure takes four symbol times for this case. During the first symbol time, the source sends $S_{1}$ to the relay, and the relay receives the signal. The received signal at the relay can be expressed as

$$
Y_{s r 1}=h_{s r} S_{1}+n_{s r 1} .
$$

During the second symbol time, the reply amplifies $Y_{s r 1}$ and forwards it to the destination. Meanwhile, the source sends $S_{2}$ to the destination simultaneously. The received signal at the destination can be written as

$$
Y_{d 2}=h_{r d} Y_{s r 1} b+h_{s d} S_{2}+n_{r d 2}
$$

where $b$ is the amplification coefficient and satisfies the power constraint, that is,

$$
b=\frac{\sqrt{\mathrm{P}}}{\sqrt{\mathrm{P}\left|\mathrm{h}_{\mathrm{sr}}\right|^{2}+\mathrm{N}_{\mathrm{o}}}}
$$

where $\mathrm{P}$ is the relay power. Again, the noise terms are modeled as the zero-mean complex Guassian random variables with variance $\mathrm{N}_{0} / 2$ per dimension. 
During the third symbol time, the source sends $\left(-S_{2}^{*}\right)$ to the relay, and the relay receives this signal. The received signal at the relay can be written as

$$
Y_{s r 3}=h_{s r}\left(-S_{2}^{*}\right)+n_{s r 3} .
$$

During the fourth symbol time, the relay amplifies $Y_{s r 3}$ and forwards it to the destination, and the source sends $S_{1}^{*}$ to the relay simultaneously. The combined signal at the destination is

$$
Y_{d 4}=h_{s d} S_{1}^{*}+h_{r d} Y_{s r 3} b+n_{r d 2}
$$

After four symbol times, the entire two blocks will be completely sent. Again, it can be assumed that the fading coefficients are not changing during the four symbol times. Chapter 4 will present bit error rate or symbol error rate performance of this scheme. Again, the procedure is shown in Table 3.2.

TABLE 3.2

\begin{tabular}{|c|c|c|}
\hline$t=1$ & $s_{1}^{s^{0}}$ & $Y_{s r 1}=h_{s r} S_{1}+n_{s r 1}$ \\
\hline$t=2$ & 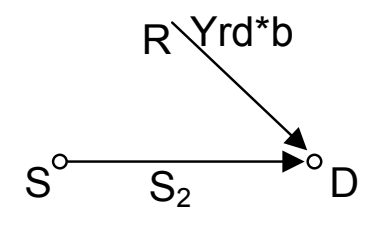 & $Y_{d 2}=h_{r d} Y_{s r 1} b+h_{s d} S_{2}+n_{r d 2}$ \\
\hline$t=3$ & $-S_{2}^{*}$ & $Y_{s r 3}=h_{s r}\left(-S_{2}^{*}\right)+n_{s r 3}$ \\
\hline $\mathrm{t}=4$ & $\mathrm{~S}_{\mathrm{S}_{1}^{*}}$ & $Y_{d 4}=h_{s d} S_{1}^{*}+h_{r d} Y_{s r 3} b+n_{r d 2}$ \\
\hline
\end{tabular}

\section{PROCEDURE OF STBC COMBINED WITH AF RELAY}




\subsubsection{Combined with DSTBC and Blind Viterbi Decoder}

The procedure was repeated, as shown in Section 3.1.2. However, it can be seen that those blind detection schemes performed poorly for the AF protocol. The reason for this is because one more fading coefficients has been introduced to the destination. The orthogonal decoding structure was destroyed. Chapter 4 will present bit error rate or symbol error rate performance of this scheme.

\subsection{Zhang's Code and Blind Viterbi Decoder under Jakes' Fading}

Jakes' fading is a Rayleigh fading associated with the Doppler spectrum. The Doppler effect is the change in frequency of a wave for an observer moving relative to the source of the wave. This means that in Jakes' fading, the channel coefficients vary based on the speed and direction of the mobile with respect to the base station.

According to Jakes [7], using fading coefficients under Jakes' fading, the channel coefficient, $y(t)$, is generated as

$$
y(t)=x_{c}(t) \cos \omega_{c} t+x_{s}(t) \sin \omega_{c} t
$$

where $x_{c}(t)=2 \sum_{n=1}^{N_{0}} \cos \beta_{n} \cos \omega_{n} t+\sqrt{2} \cos \alpha t \cos \omega_{m} t, \quad x_{s}(t)=2 \sum_{n=1}^{N_{0}} \sin \beta_{n} \cos \omega_{n} t+$ $\sqrt{2} \sin \alpha \cos \omega_{m} t, N_{0}$ is the low-frequency oscillator with frequencies equal to the Doppler shifts, that is,

$$
\omega_{n}=\omega_{m} \cos \frac{2 \pi n}{N}
$$

where $\omega_{m}=2 \pi \frac{V}{V_{c}} f_{c}, \mathrm{~N}=1,2, \ldots, N_{0}, V$ is the mobile speed, $V_{c}$ is the speed of the light, and $f_{c}$ is the carry frequency. In this thesis, the following was chosen: $N_{0}=8$, and $\alpha=\frac{\pi}{4}$. 


\section{CHAPTER 4}

\section{SIMULATION RESULTS}

\subsection{Simulation Parameters}

This section shows the performance of the relay system combined with the STBC coherent detection scheme and blind detection schemes, i.e., DSTBC, the blind Viterbi, and Zhang's code. Also, the performance of the different relay location is presented. All simulation results were obtained using $1,000,000$ bits, and 200 symbols per frame. During those 200 symbols, the fading coefficients, $h_{s d}, h_{r d}$, and $h_{s r}$, were assumed to be constant. The BPSK or QPSK modulations were considered. It was also assumed that the distance (Dsd) between the source and the destination is normalized to 1 , and the transmitted signal has unit energy. The simulation code are shown in Appendix A, B, C, D, E, F and G.

\subsection{DF Relay Combined with STBC}

Figure 4.1 shows BER performance of a single-relay system with the DF protocol when coherent STBC detection is used. 


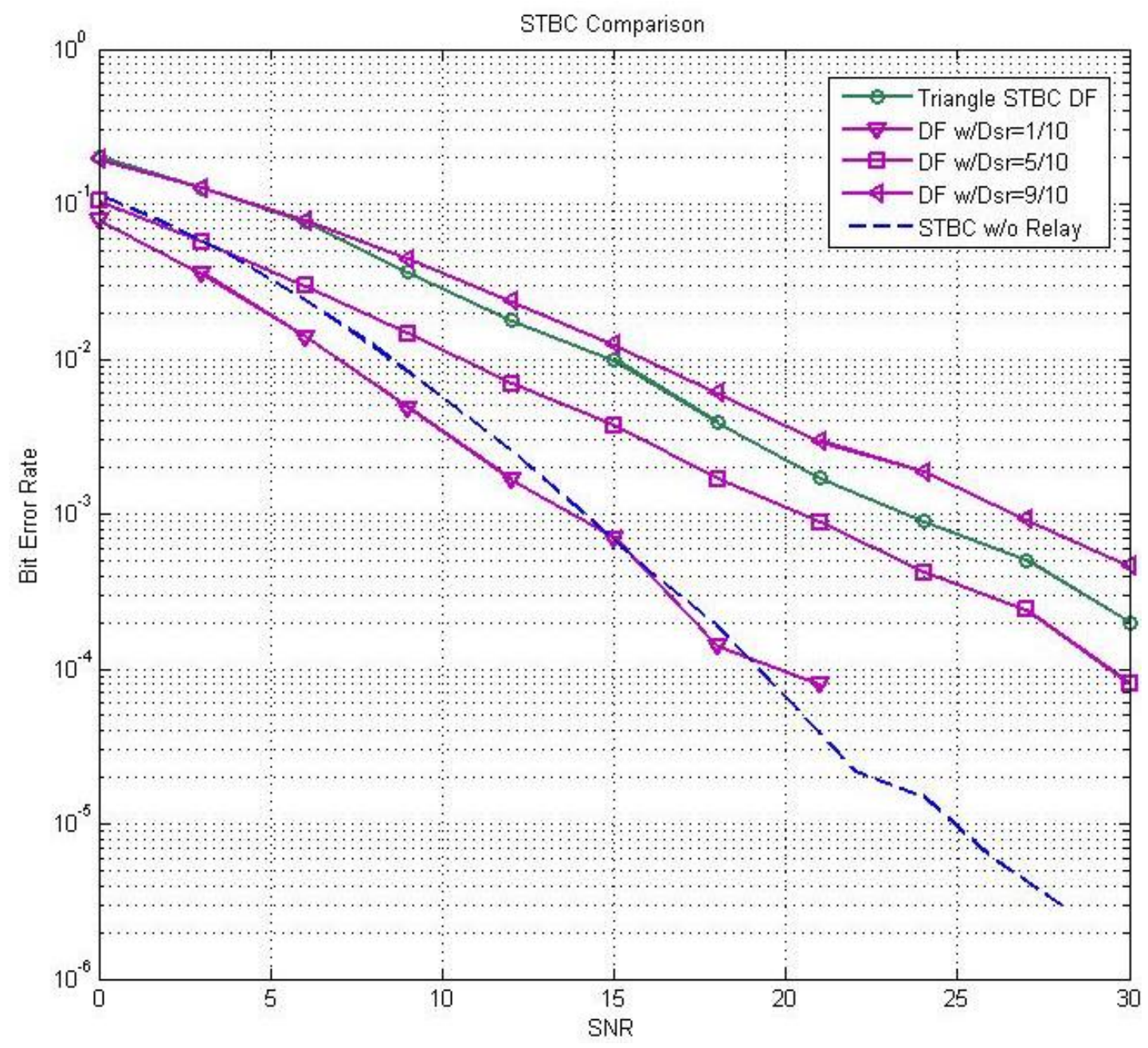

Figure 4.1. BER performance of single relay system with DF protocol when coherent STBC detection is used.

\subsection{DF Relay Combined with DSTBC}

Figure 4.2 shows BER performance of a single-relay system with the DF protocol when the noncoherent DSTBC detection is used. 


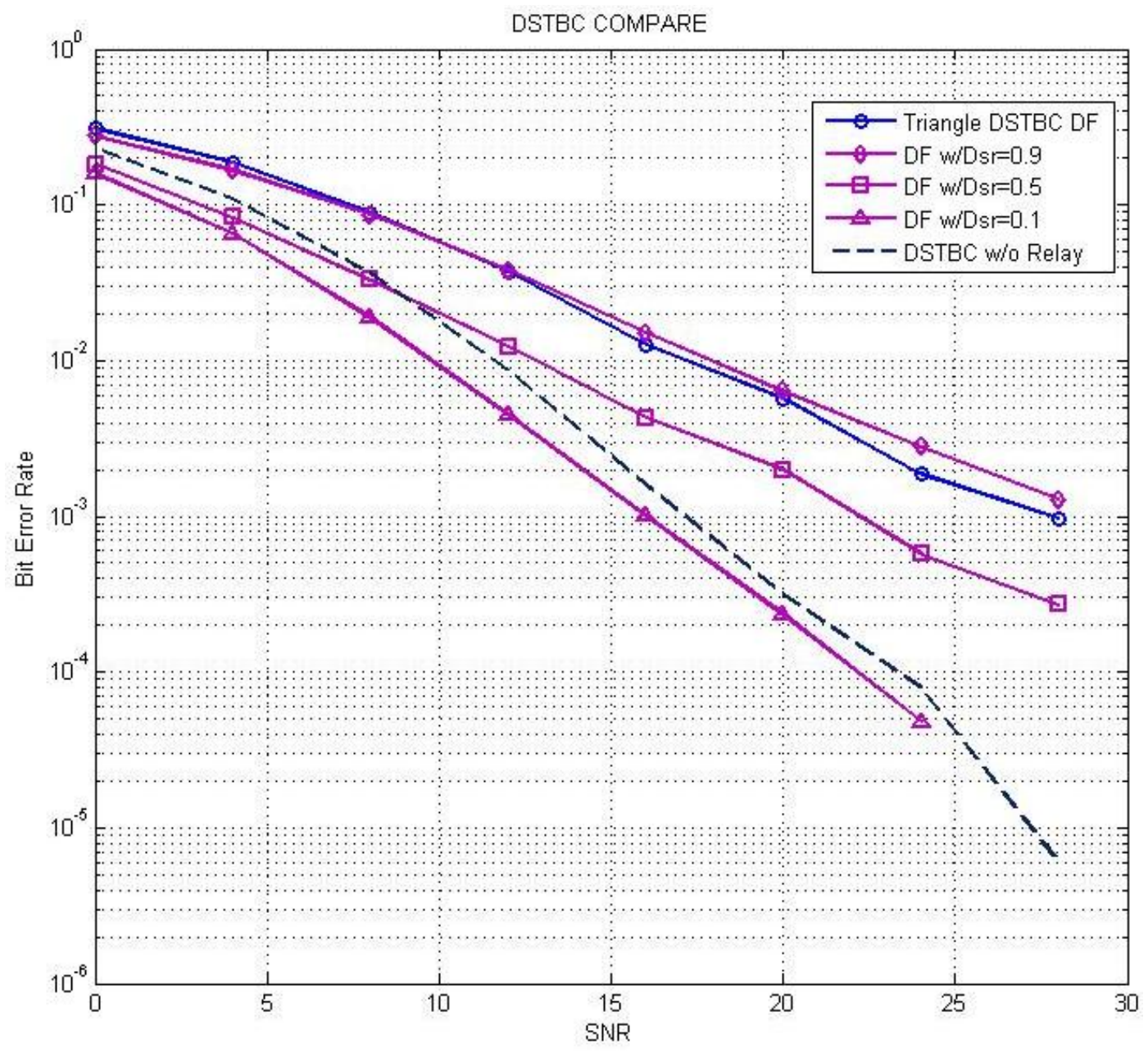

Figure 4.2. BER performance of single-relay system with DF protocol when noncoherent DSTBC detection is used.

\subsection{DF Relay Combined with Blind Viterbi Decoder}

Figure 4.3 shows BER performance of the single-relay system with the DF protocol when the noncoherent blind Viterbi decoder is used.

Figures $4.1,4.2$, and 4.3 show that regardless of using coherent or noncoherent detection schemes, when the relay is closer to the source, performance is better for the parallel relay, as shown in Figure 3.2. If the relay is getting closer to the destination, then the performance is closer to that of the simple triangle relay, as shown in Figure 3.1 


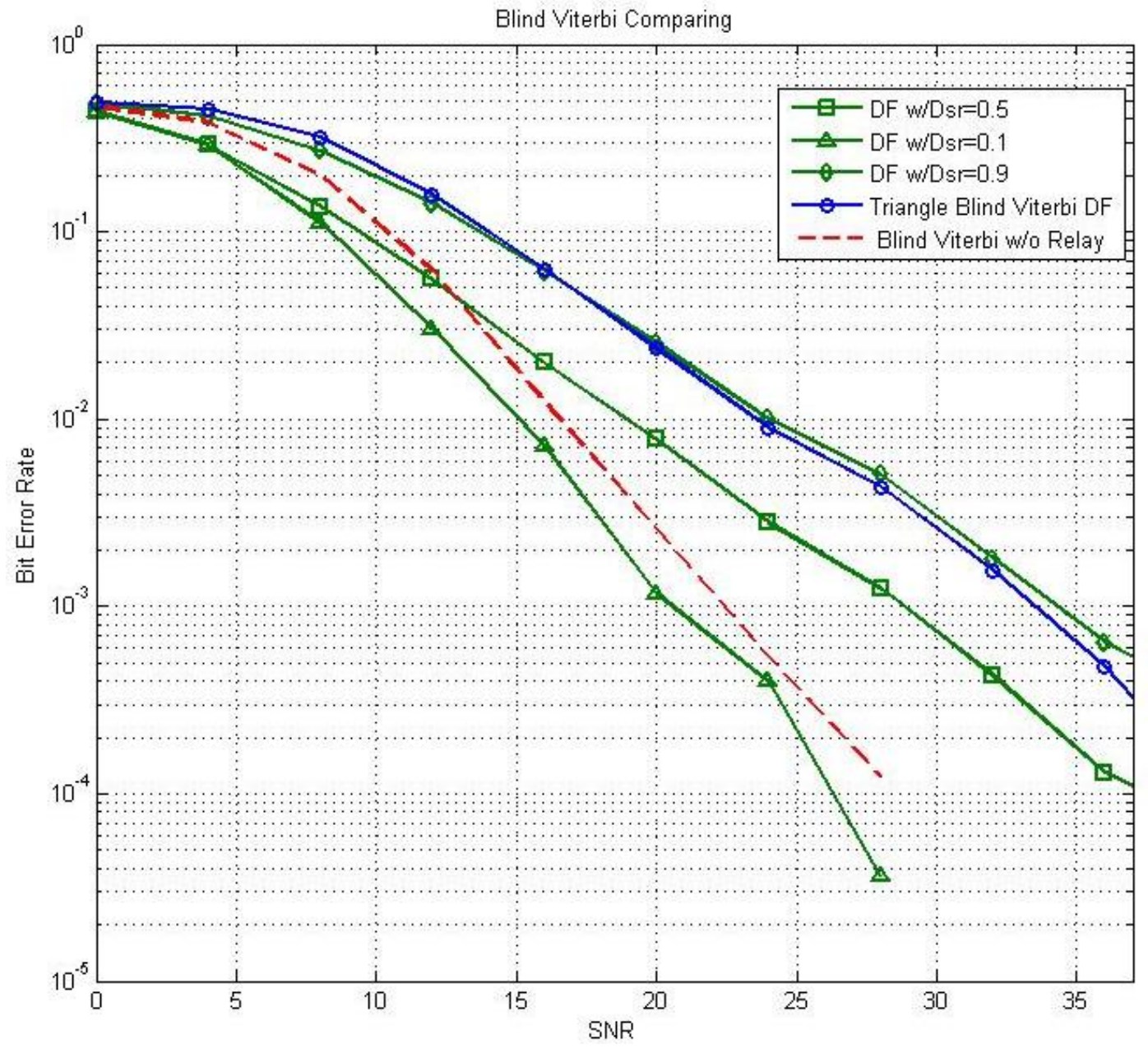

Figure 4.3. BER performance of single relay system with DF protocol when the noncoherent blind Viterbi decoder is used.

\subsection{Single AF Relay}

Figure 4.4 shows BER performance of the single-relay system with the AF protocol when the STBC, DSTBC, and blind Viterbi decoder are used. It can be seen that when noncoherent detection schemes combine with the AF single relay, performance becomes unacceptable. This is because orthogonality is destroyed. 


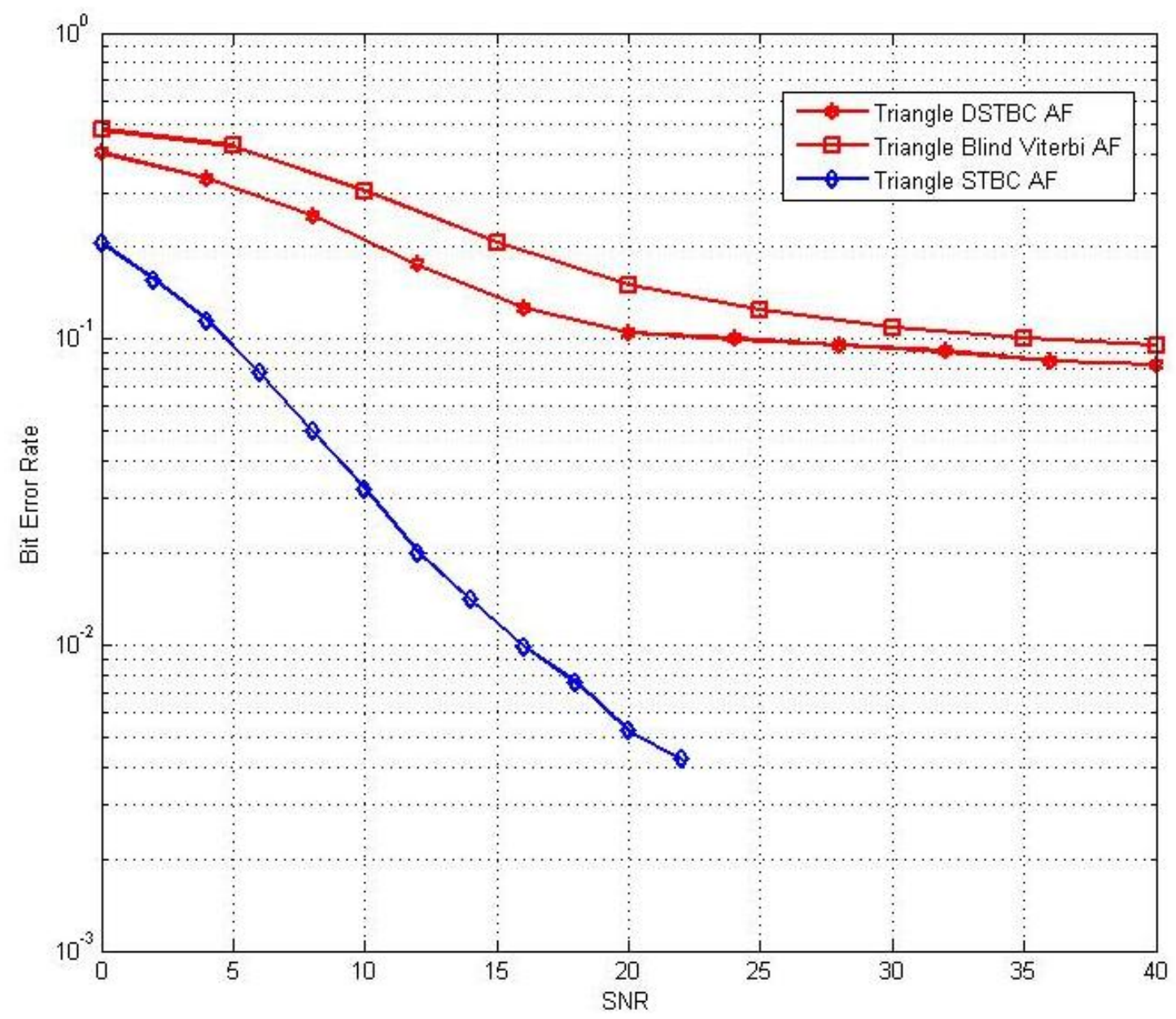

Figure 4.4. BER performance of single relay system with AF protocol when STBC, DSTBC, and blind Viterbi decoder are used.

\subsection{SER Performance of Zhang's Code and Blind Viterbi}

Figure 4.5 shows SER performance of Zhang's code and the blind Viterbi decoder under a Jakes' fading case when fdTs $=0.01$. As shown, the Zhang's code is worse than the blind Viterbi decoder, when the four-block length is used. It can be assumed that the fading coefficients are not changing during the four blocks, and the Zhang's code uses the 3PSK and QPSK for its encoder. Also note that in the case of the QPSK blind Viterbi decoder, the performance is better than with BPSK. This is due to the redundancy introduced in the coefficient vector set $\mathrm{V}$. 


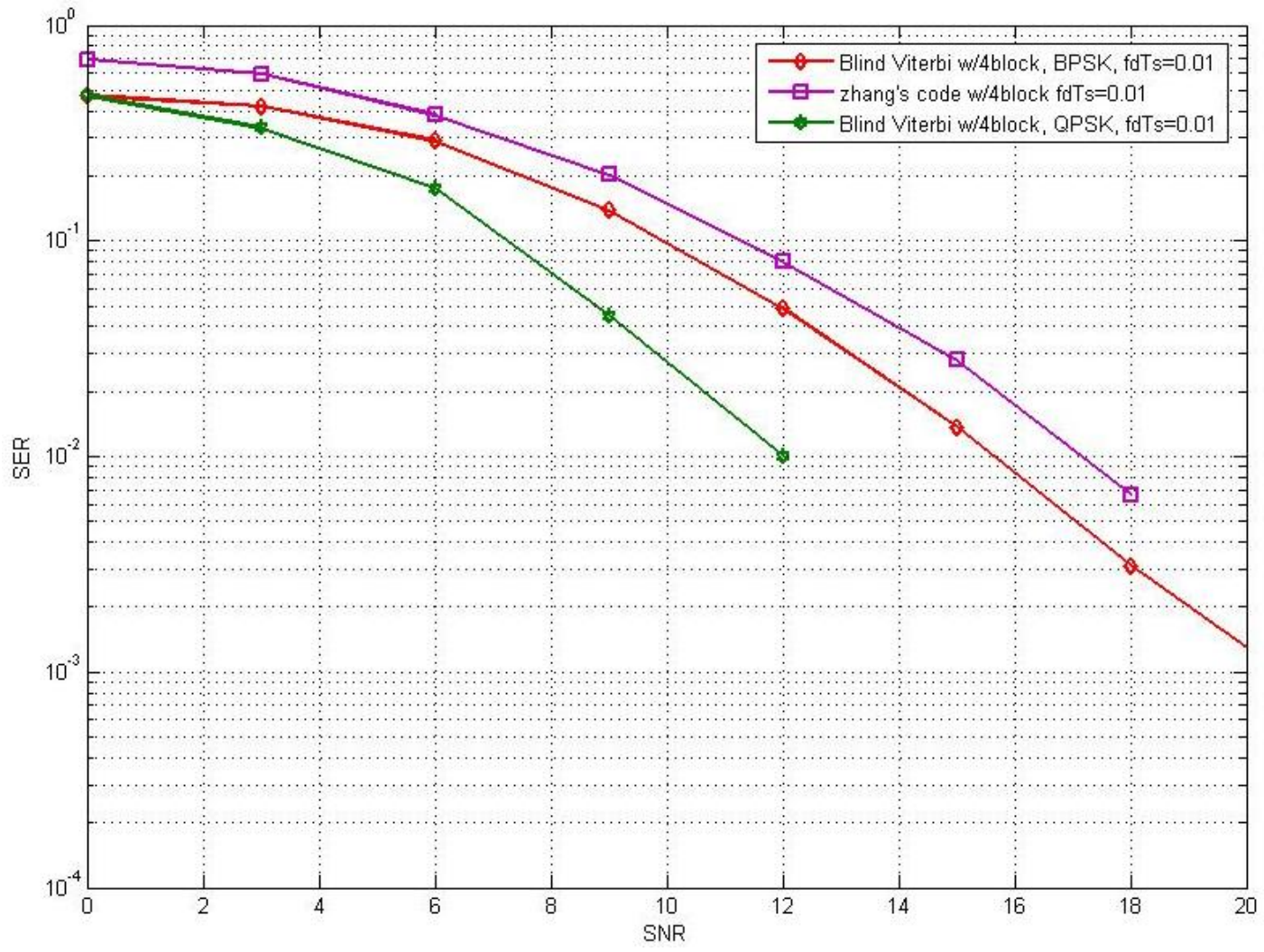

Figure 4.5. BER performance of Zhang's code and blind Viterbi decoder under Jakes' fading case. 


\section{CHAPTER 5}

\section{CONCLUSION}

\subsection{Conclusion}

Simulation results shows that the blind detection schemes did not work with the amplify-and-forward relay protocol. This is because one more fading coefficient was introduced to the decoder, thus destroying orthogonality. It was observed that the estimation equations at the decoder for the DSTBC and the blind Viterbi decoder contained two fading coefficients. This means that introducing one more fading coefficient would destroy the orthogonal decoding structure.

However, it was also observed that the blind detection schemes with the DF protocol performed well. The reason for this is because estimation was performed at the relay. Again, it was noticed that in the case of the parallel relay, the closer the relay to the source, the better the performance.

For Zhang's code and the blind Viterbi decoder under Jakes fading, assuming that Zhang's code uses four blocks as the blind Viterbi decoder, it was observed that using Zhang's code was $1 \mathrm{~dB}$ worse than using the BPSK blind Viterbi decoder, and 44.5 dB worse than using the QPSK blind Viterbi decoder. This is because redundancy was introduced by the encoder for the QPSK or higher modulation. 
REFERENCES 


\section{REFERENCES}

[1] K. J. Ray Lui, Ahmed Sadek, Weifeng Su, and Andres Kwasiski, Cooperative Communications and Networking, Cambridge University Press, 2009.

[2] V. Tarokh and H. Jafarkhani, "A Differential Detection Scheme for Transmit Diversity," IEEE Journal on Selected Areas in Comm., Vol. 18, No. 7, pp. 11691174, July 2000.

[3] X. Shao, J. Yuan, Y. Hong, and M. N. Patwary, "Blind Detection of Space-Time Block Codes and Application for Iterative Receiver," 2003, Proceedings of IEEE Vehicular Technology Conference, October 9, 2003, Vol. 5, pp. 523-526

[4] J. Zhang and W. Ma, "Full Diversity Blind Alamouti Space-Time Block Codes for Unique Identification of Flat-Fading Channels," IEEE Transactions on Signal Processing, February 2009, Vol. 57, No. 2, pp. 653-644

[5] S. M. Alamouti, "A Simple Transmit Diversity Technique for Wireless Communications," IEEE Journal on Selected Areas in Comm., Vol. 16, No. 8, pp. 1451-1458, Oct. 1998.

[6] V. Tarokh, S. M. Alamouti, and P. Poon, "New Detection Schemes for Trasmit Diversity with No Channel Estimation," Proceeding of ICUPC' 98, Oct. 1998, Vol. 2, pp. 917-920.

[7] W. C. Jakes, Microwave Mobile Communications, Wiley-IEEE Press, 1994. 


\section{APPENDIXES}




\section{APPENDIX A}

PROGRAM OF DF RELAY COMBINED WITH STBC

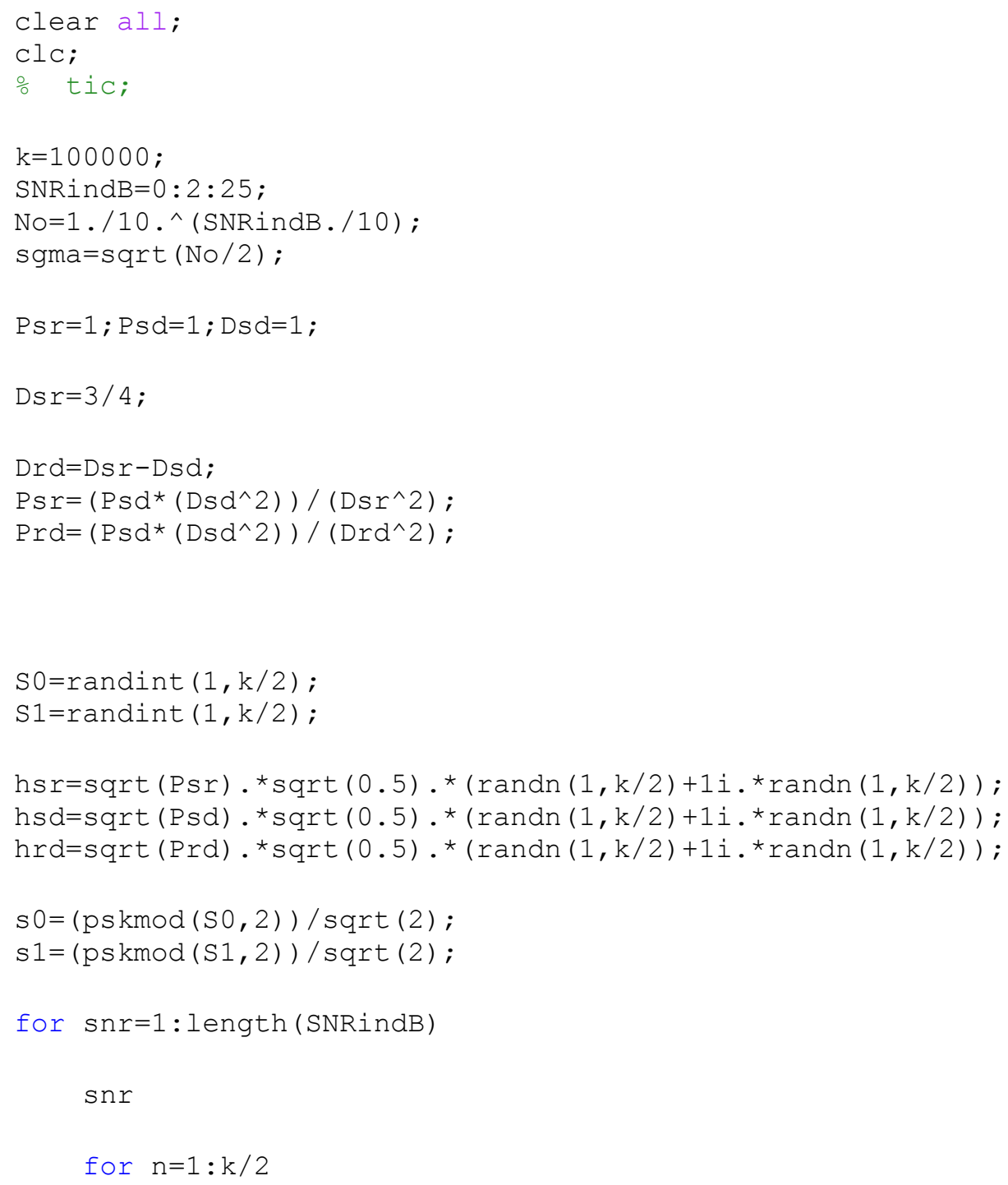




\section{APPENDIX A (Continued)}

$r 1(n)=(-$

$\operatorname{conj}(\operatorname{ysr} 2(\mathrm{n}))) * \operatorname{hrd}(\mathrm{n})+\operatorname{hsd}(\mathrm{n}) *(\operatorname{conj}(\operatorname{so}(\mathrm{n})))+\operatorname{sgma}(\operatorname{snr}) *(\operatorname{randn}+1$ i*randn $)$;

s_O (n) $=\operatorname{conj}(\mathrm{hrd}(\mathrm{n})){ }^{*} \mathrm{r} 0(\mathrm{n})+\mathrm{hsd}(\mathrm{n}){ }^{*} \operatorname{conj}(\mathrm{rl}(\mathrm{n}))$;

$s_{-} 1(n)=\operatorname{conj}($ hsd $(n)) * r 0(n)-h r d(n) * \operatorname{conj}(r 1(n))$;

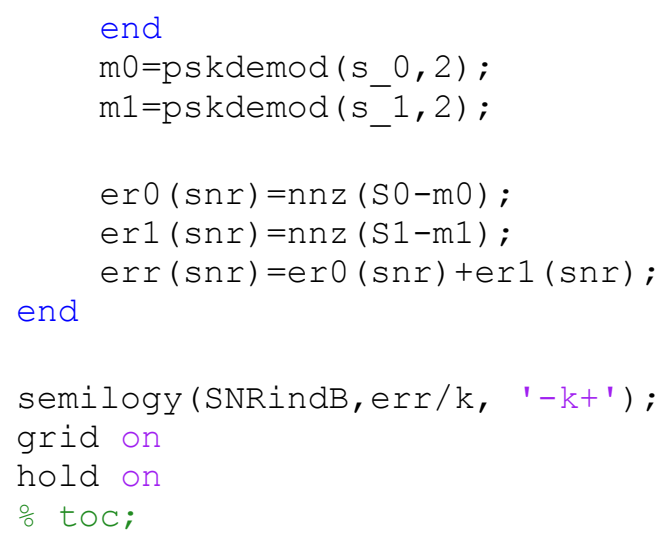




\section{APPENDIX B}

\section{PROGRAM OF DF RELAY COMBINED WITH DSTBC}

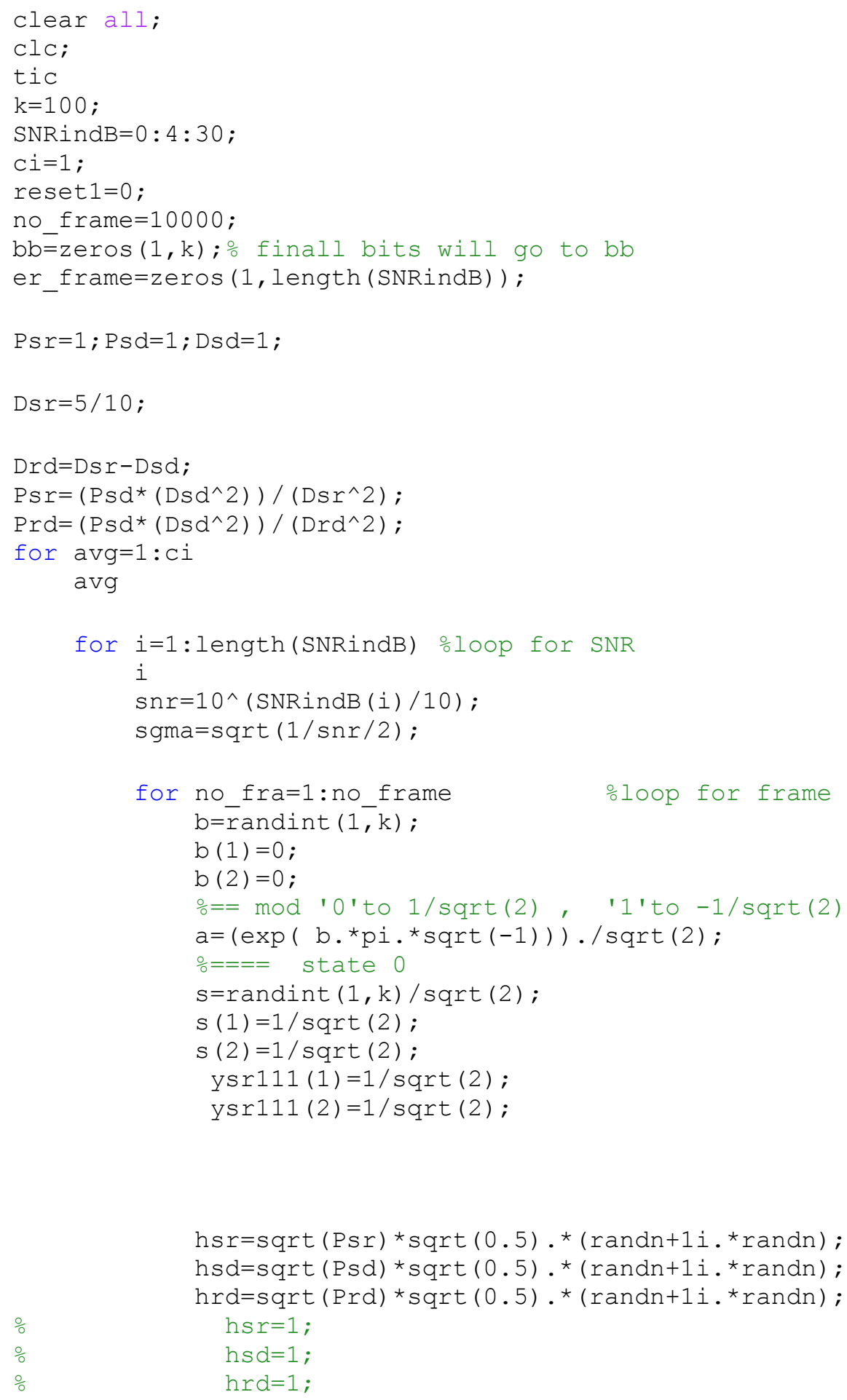




\section{APPENDIX B (Continued)}

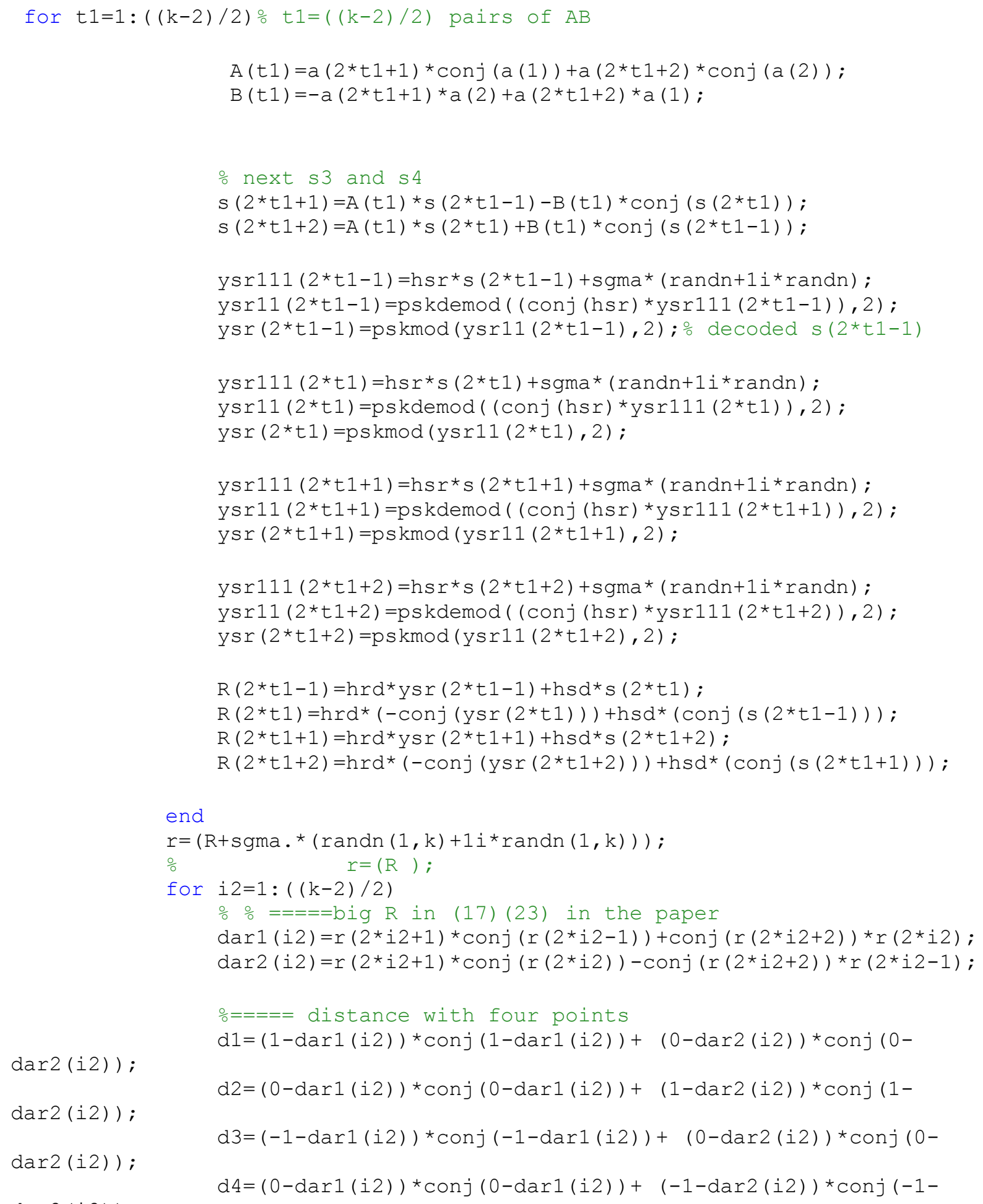




\section{APPENDIX B (Continued)}

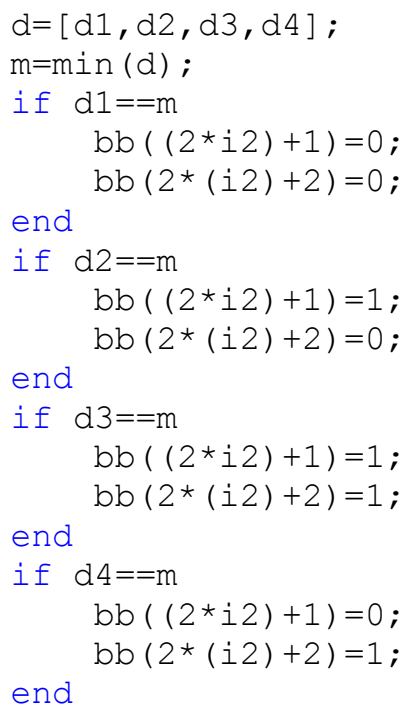




\section{APPENDIX C}

\section{PROGRAM OF DF RELAY COMBINED WITH BLIND VITERBI DECODER}

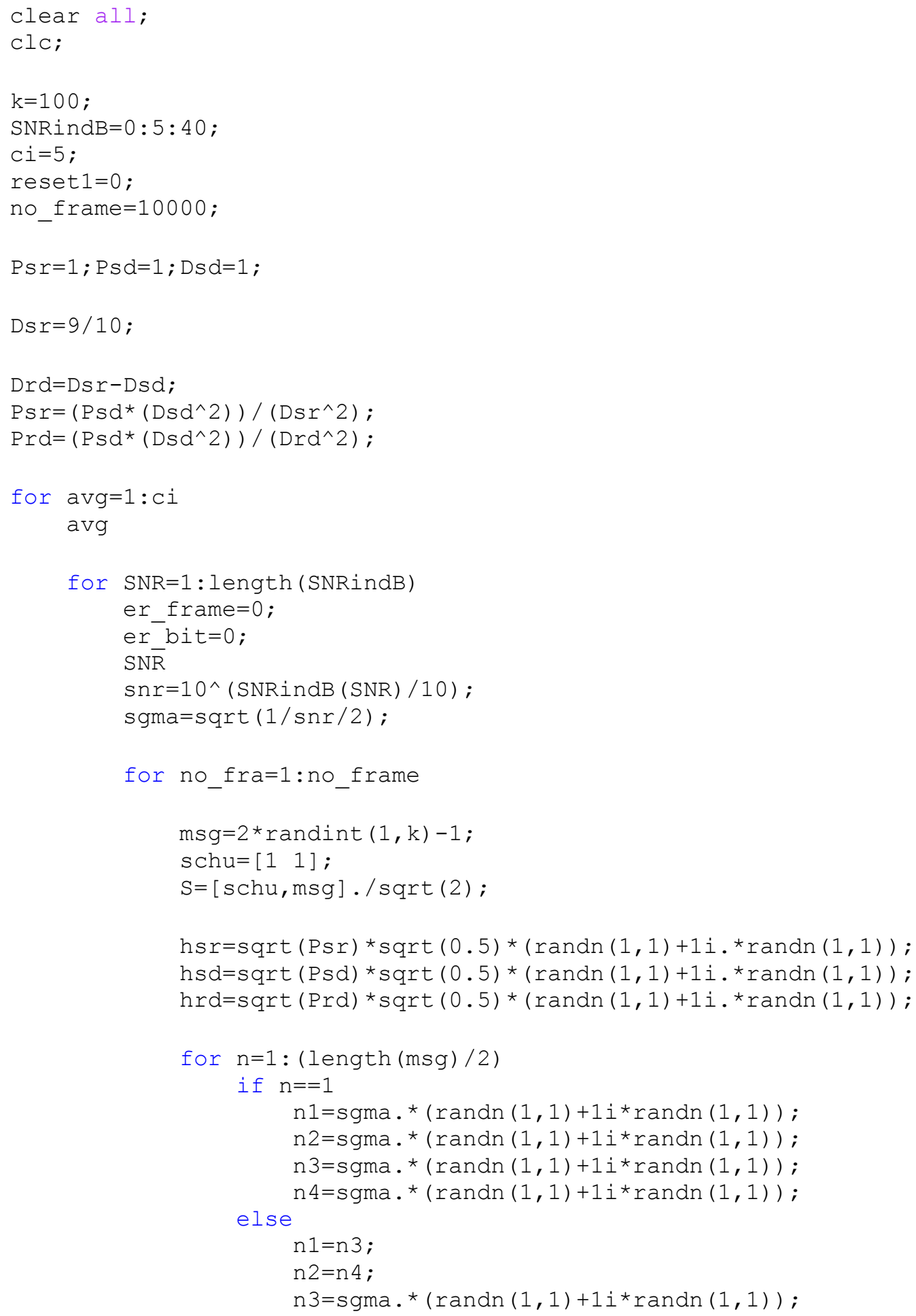




\section{APPENDIX C (Continued)}

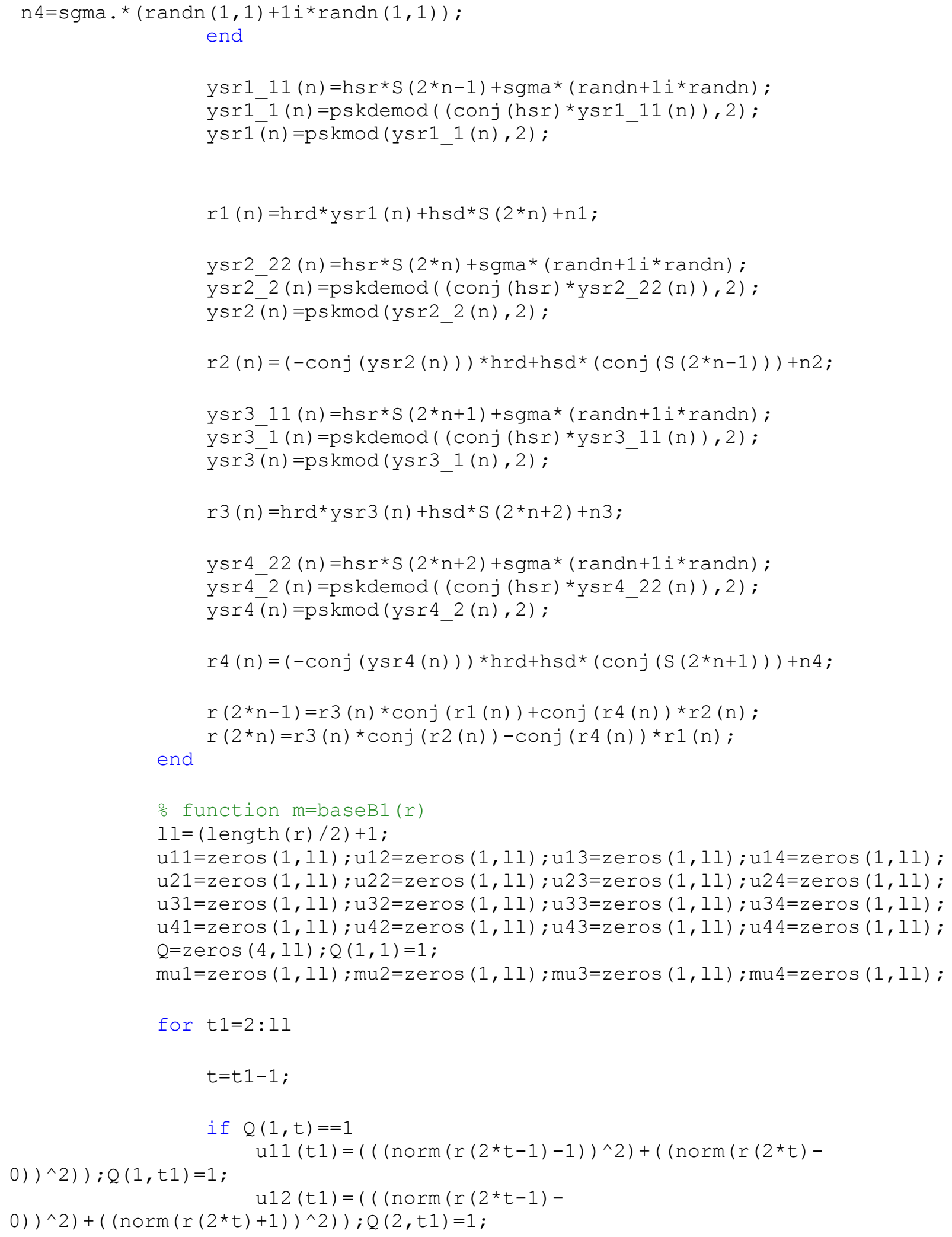




\section{APPENDIX C (Continued)}

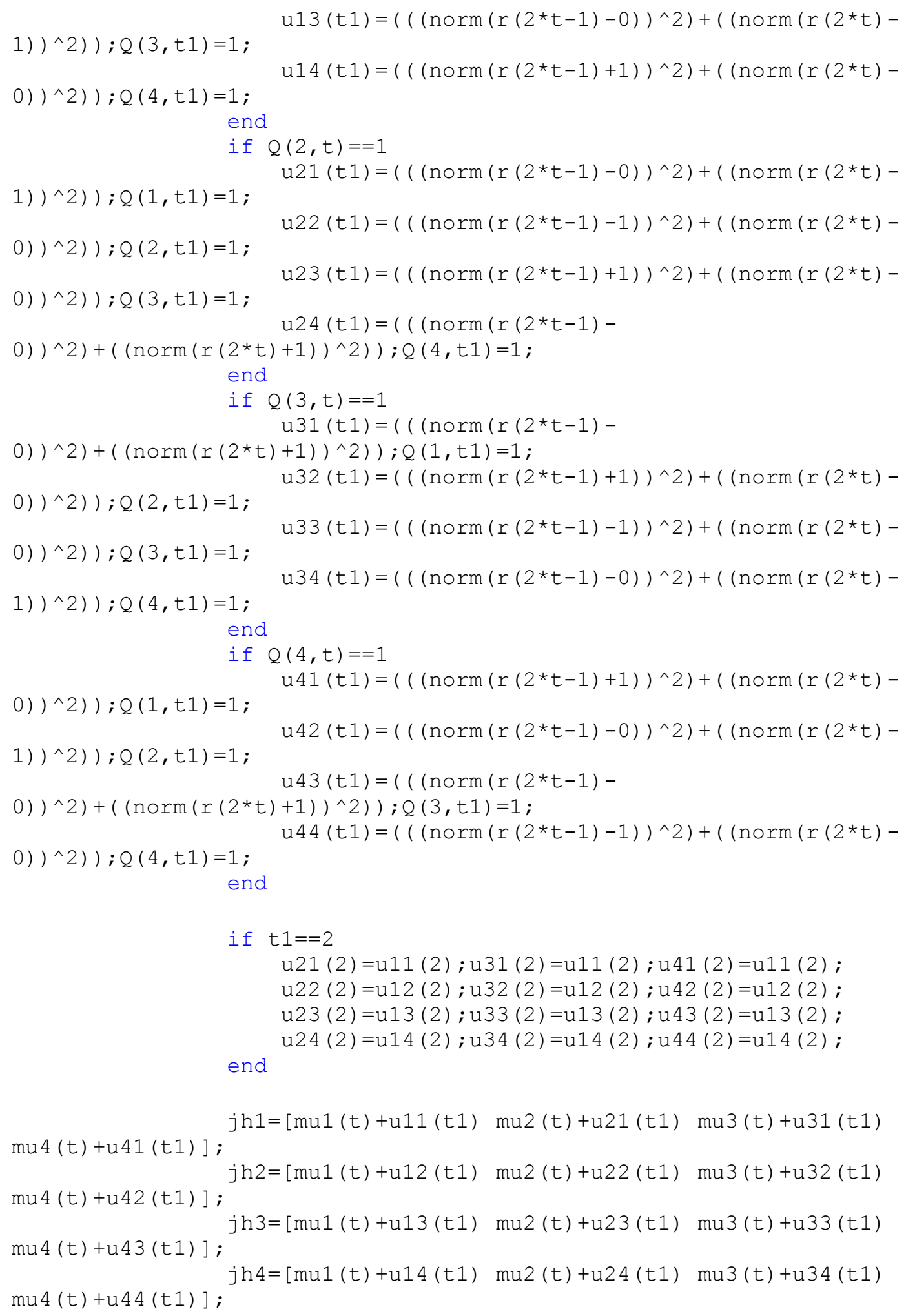




\section{APPENDIX C (Continued)}

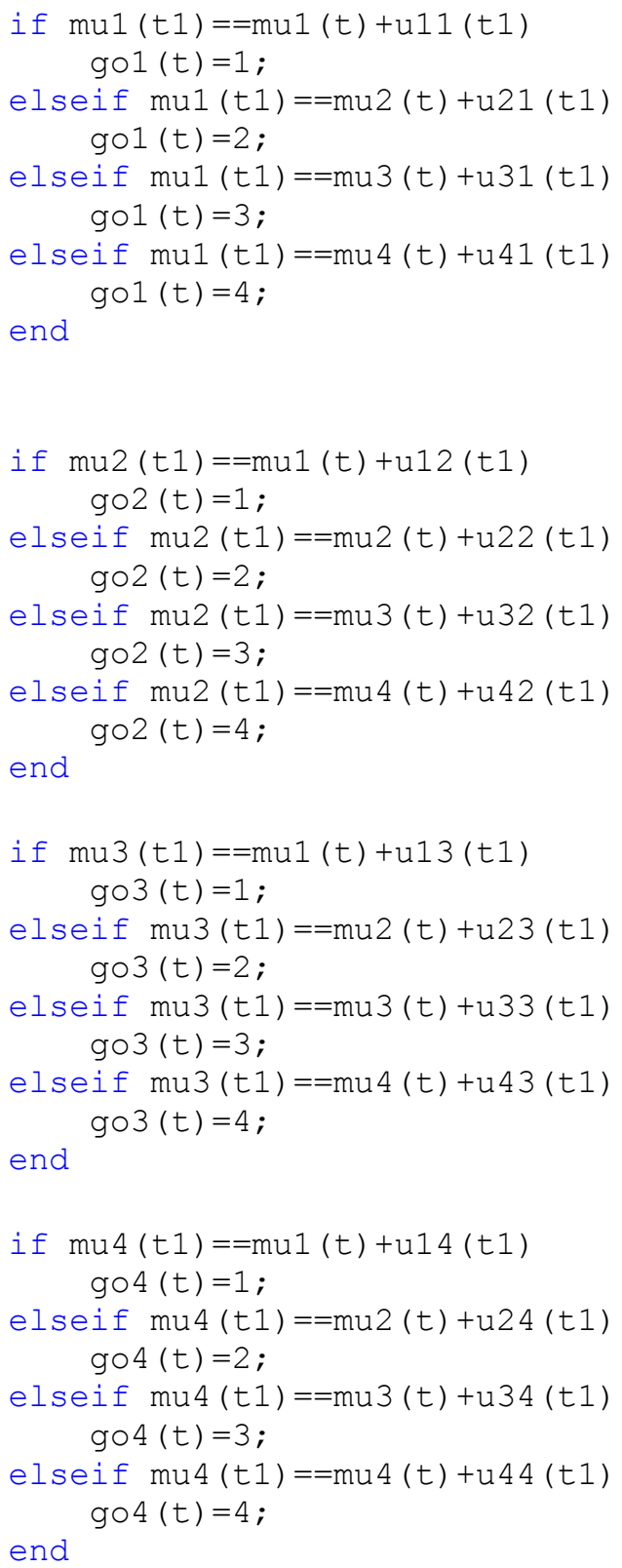




\section{APPENDIX C (Continued)}

$11($ length $(g \circ 1))=g \circ(1$, length $(g \circ 1))$;

for $t 2=$ length $(g \circ 1):-1: 2$

$11(t 2-1)=g \circ(11(t 2), t 2-1)$;

if $l 1(t 2)==1$

$\mathrm{m}(2 * t 2-3)=1 ; \mathrm{m}(2 * t 2-2)=1$;

elseif $11(t 2)==2$

$\mathrm{m}(2 * t 2-3)=1 ; \mathrm{m}(2 * t 2-2)=-1$;

elseif $11(t 2)==3$

$m(2 * t 2-3)=-1 ; m(2 * t 2-2)=1$;

elseif $11(t 2)==4$

$\mathrm{m}(2 \star t 2-3)=-1 ; \mathrm{m}(2 \star t 2-2)=-1 ;$

end

end

elseif start $==\operatorname{mu} 2(11)$

$11($ length $(g \circ 1)+1)=2$;

$\mathrm{m}(2 *$ length $(\mathrm{g \circ 1})-1)=1 ; \mathrm{m}(2 *$ length $(\mathrm{g} \circ 1))=-1$;

$11($ length $(g \circ 1))=g \circ(2$, length $(g \circ 1))$;

for t2=length $(g \circ 1):-1: 2$

$11(t 2-1)=g \circ(11(t 2), t 2-1)$;

if $11(t 2)==1$

$\mathrm{m}(2 * t 2-3)=1 ; \mathrm{m}(2 * t 2-2)=1$;

elseif $11(t 2)==2$

$m(2 * t 2-3)=1 ; m(2 * t 2-2)=-1 ;$

elseif $11(t 2)==3$

$\mathrm{m}(2 * t 2-3)=-1 ; \mathrm{m}(2 * t 2-2)=1 ;$

elseif $11(t 2)==4$

$m(2 * t 2-3)=-1 ; m(2 * t 2-2)=-1 ;$

end

end

elseif start $==\operatorname{mu} 3(11)$

$11($ length $(g \circ 1)+1)=3$;

$\mathrm{m}(2 *$ length $(\mathrm{g} \circ 1)-1)=-1 ; \mathrm{m}(2 *$ length $(\mathrm{g} \circ 1))=1$;

$11($ length $($ gol $))=g \circ(3$, length $(g \circ 1))$;

for $t 2=$ length $(g \circ 1):-1: 2$

$11(t 2-1)=g \circ(11(t 2), t 2-1)$;

if $l 1($ t 2$)==1$

$\mathrm{m}(2 \star t 2-3)=1 ; \mathrm{m}(2 \star t 2-2)=1 ;$

elseif $11(t 2)==2$

$\mathrm{m}(2 * t 2-3)=1 ; \mathrm{m}(2 * t 2-2)=-1 ;$

elseif $11(t 2)==3$

$m(2 * t 2-3)=-1 ; m(2 * t 2-2)=1 ;$

elseif $11(t 2)==4$

$m(2 * t 2-3)=-1 ; m(2 * t 2-2)=-1 ;$

end

end

elseif start $==$ mu4 (ll)

$11($ length $(g \circ 1)+1)=4$;

$m(2 *$ length $(g \circ 1)-1)=-1 ; m(2 *$ length $(g \circ 1))=-1$;

$11($ length $(g \circ 1))=g \circ(4$, length $(g \circ 1))$;

for $t 2=$ length $(g \circ 1):-1: 2$

$11(t 2-1)=g \circ(11(t 2), t 2-1)$; 


\section{APPENDIX C (Continued)}

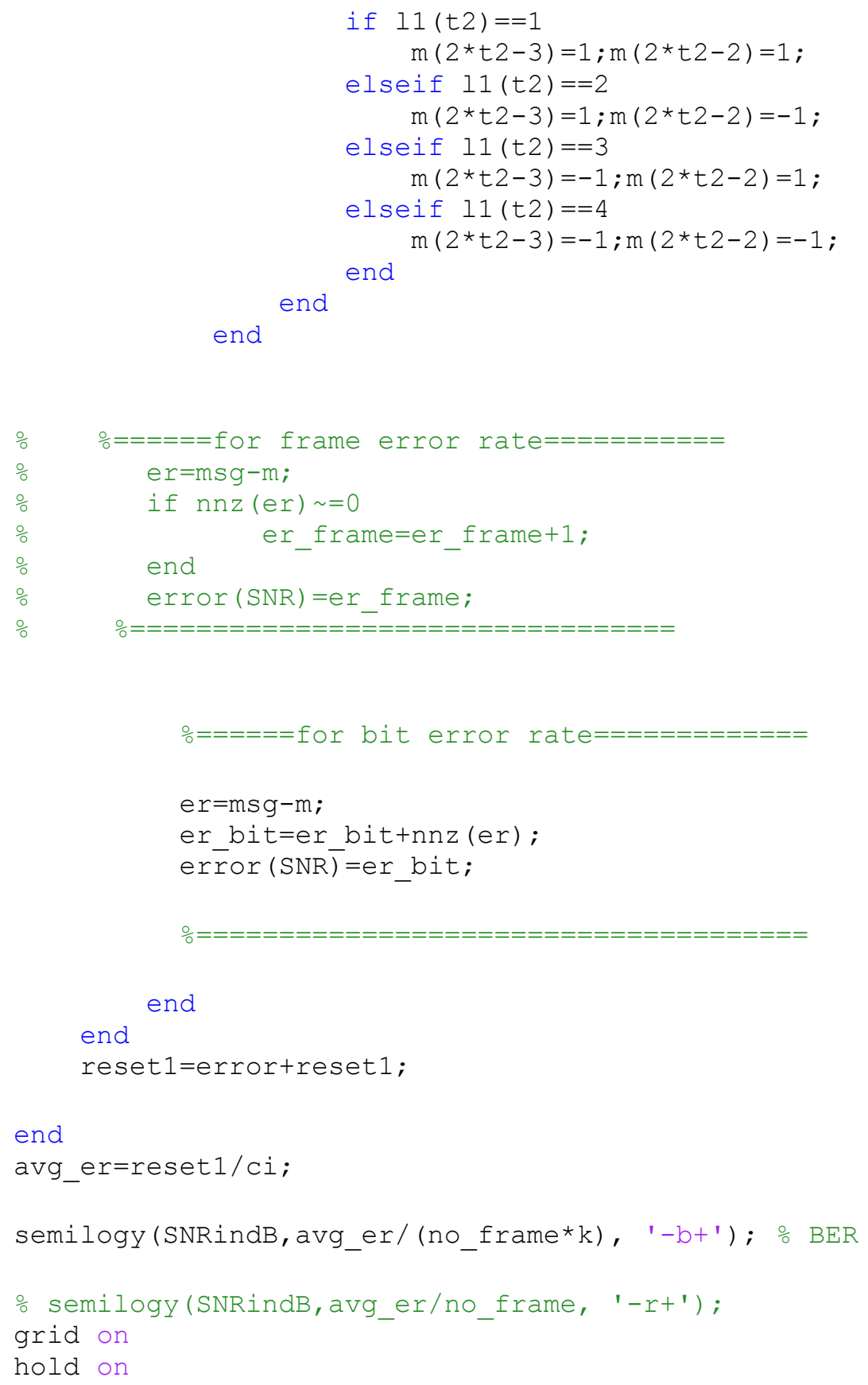




\section{APPENDIX D}

\section{PROGRAM OF BLIND VITERBI COMBINED WITH AF}

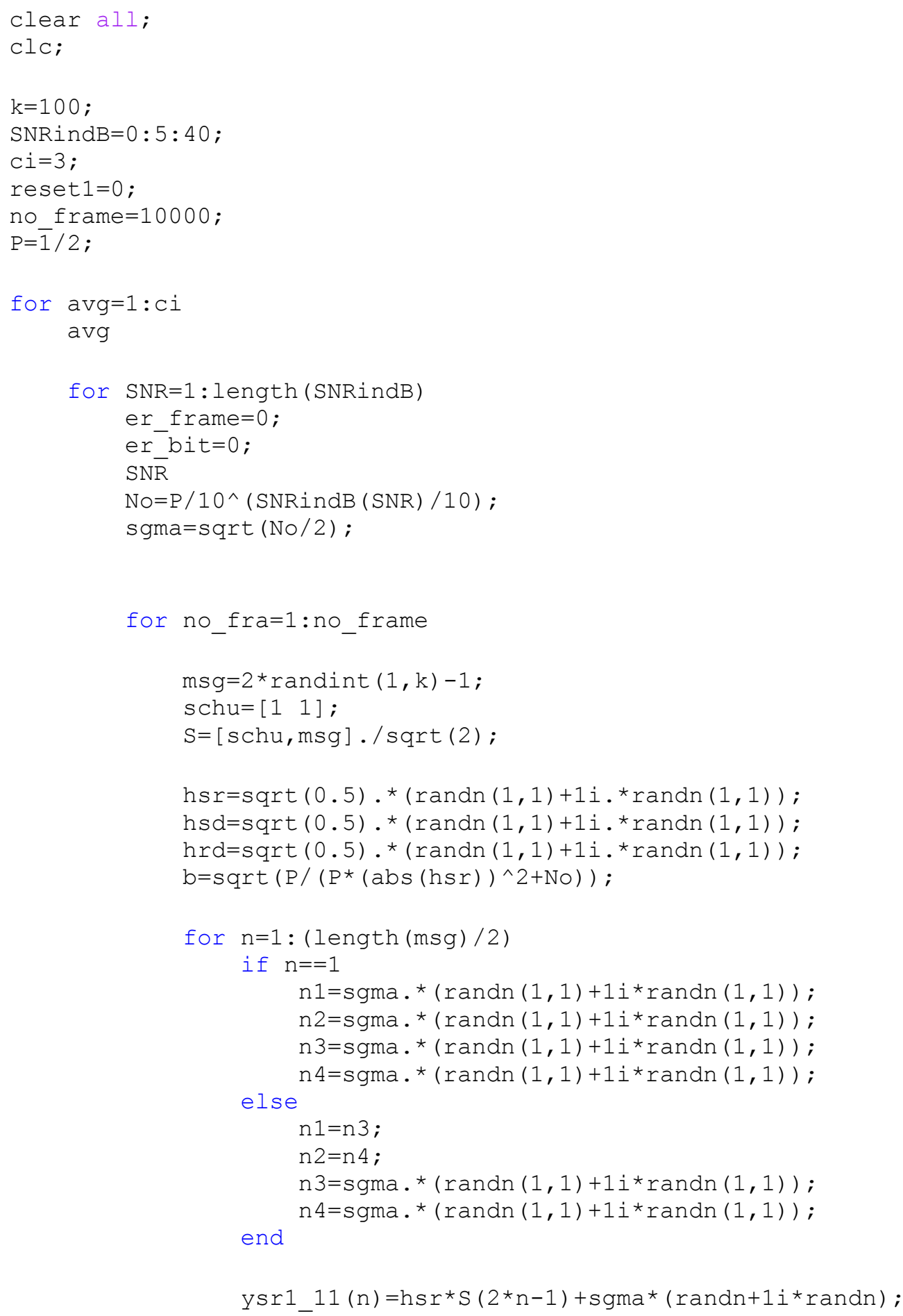




\section{APPENDIX D (Continued)}

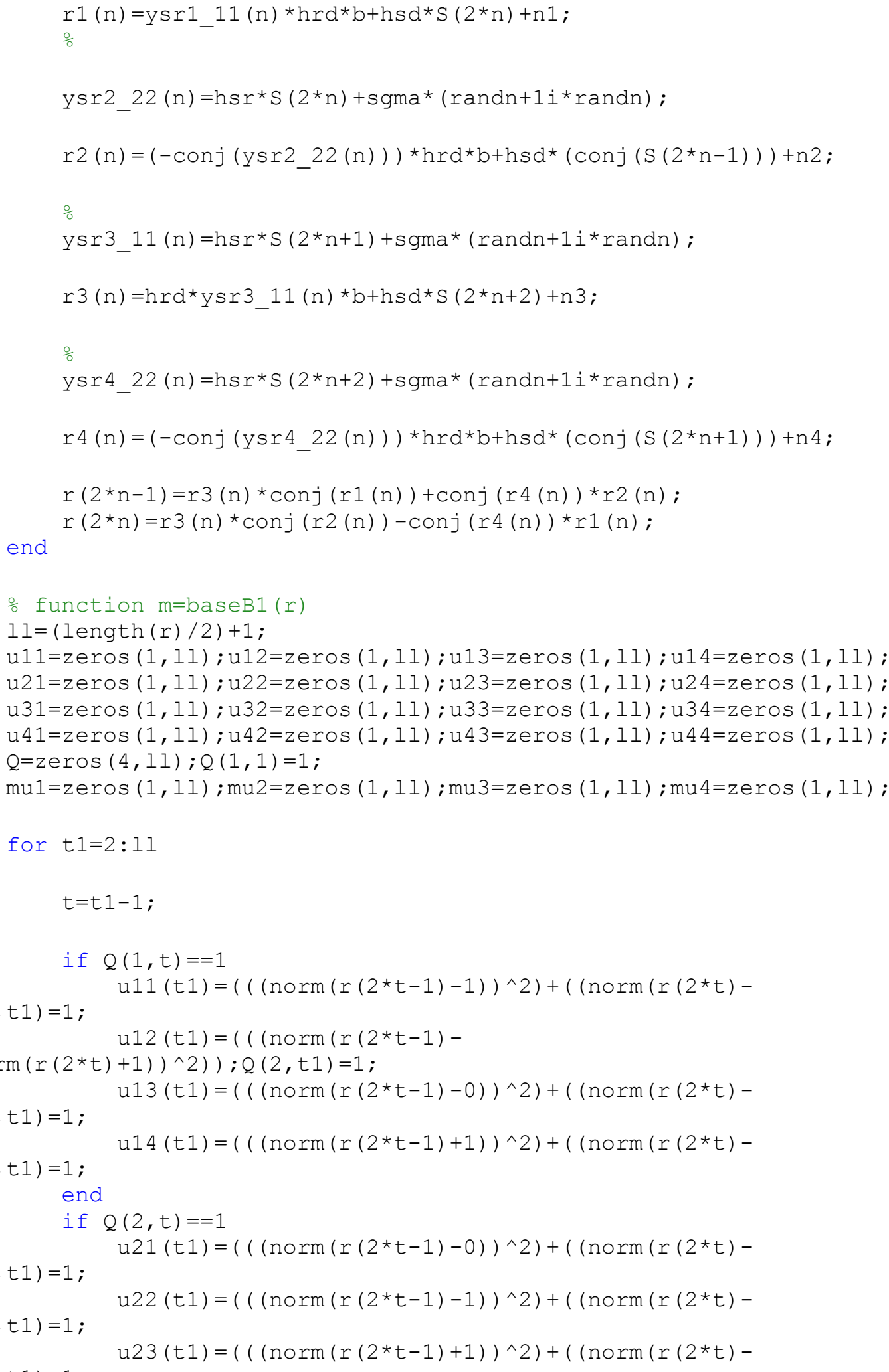

\section{APPENDIX C (Contiuned)}




\section{APPENDIX D (Continued)}

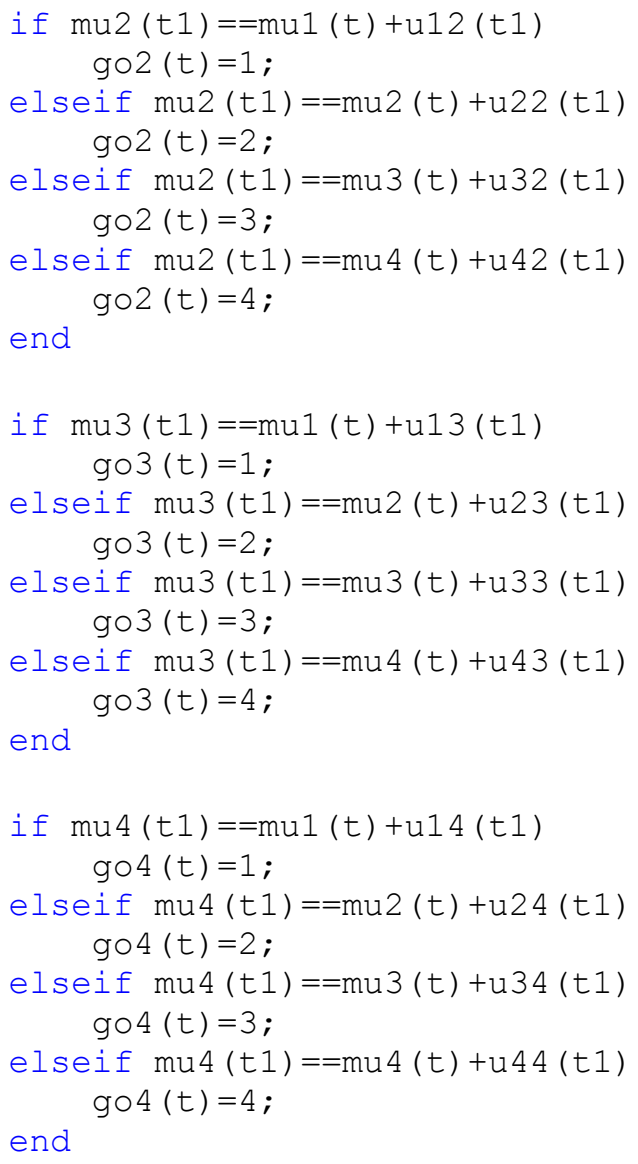

end

if $\operatorname{mu} 4(t 1)==\operatorname{mul}(t)+u 14(t 1)$ go4 $(t)=1$;

elseif mu4(t1) $==\operatorname{mu} 2(t)+u 24(t 1)$ $g \circ 4(t)=2$;

elseif mu4 (t1) $==\operatorname{mu} 3(t)+u 34(t 1)$ $g \circ 4(t)=3$;

elseif mu4 (t1) $==\operatorname{mu} 4(t)+u 44(t 1)$ $g \circ 4(t)=4$;

end

end

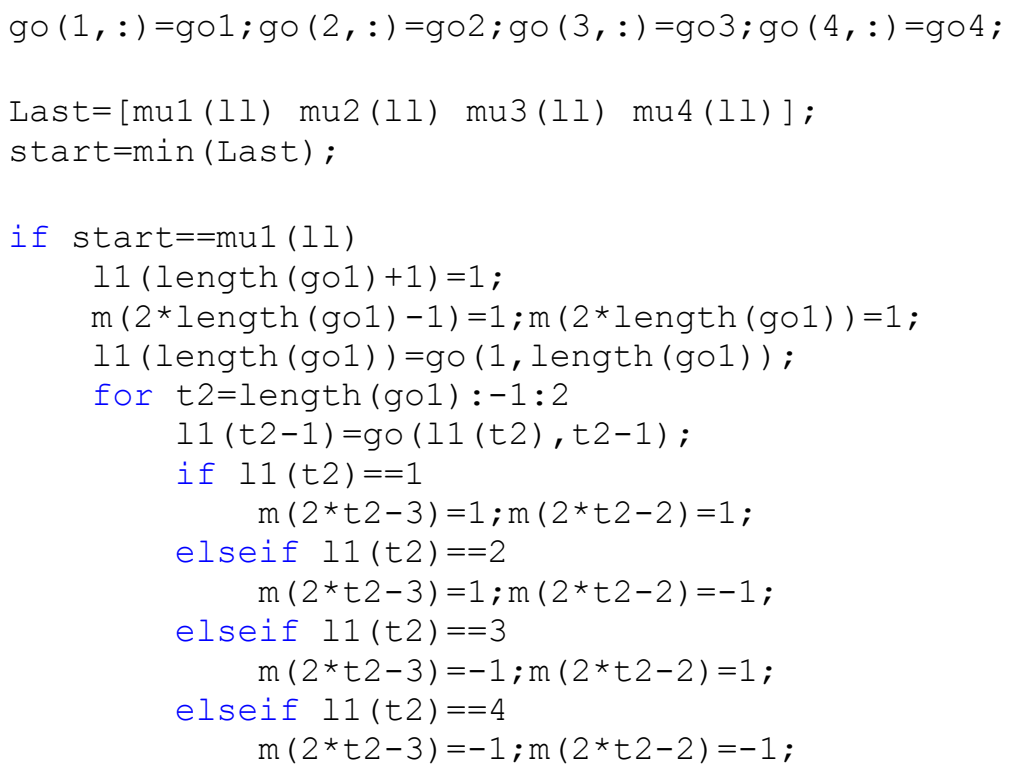




\section{APPENDIX D (Continued)}

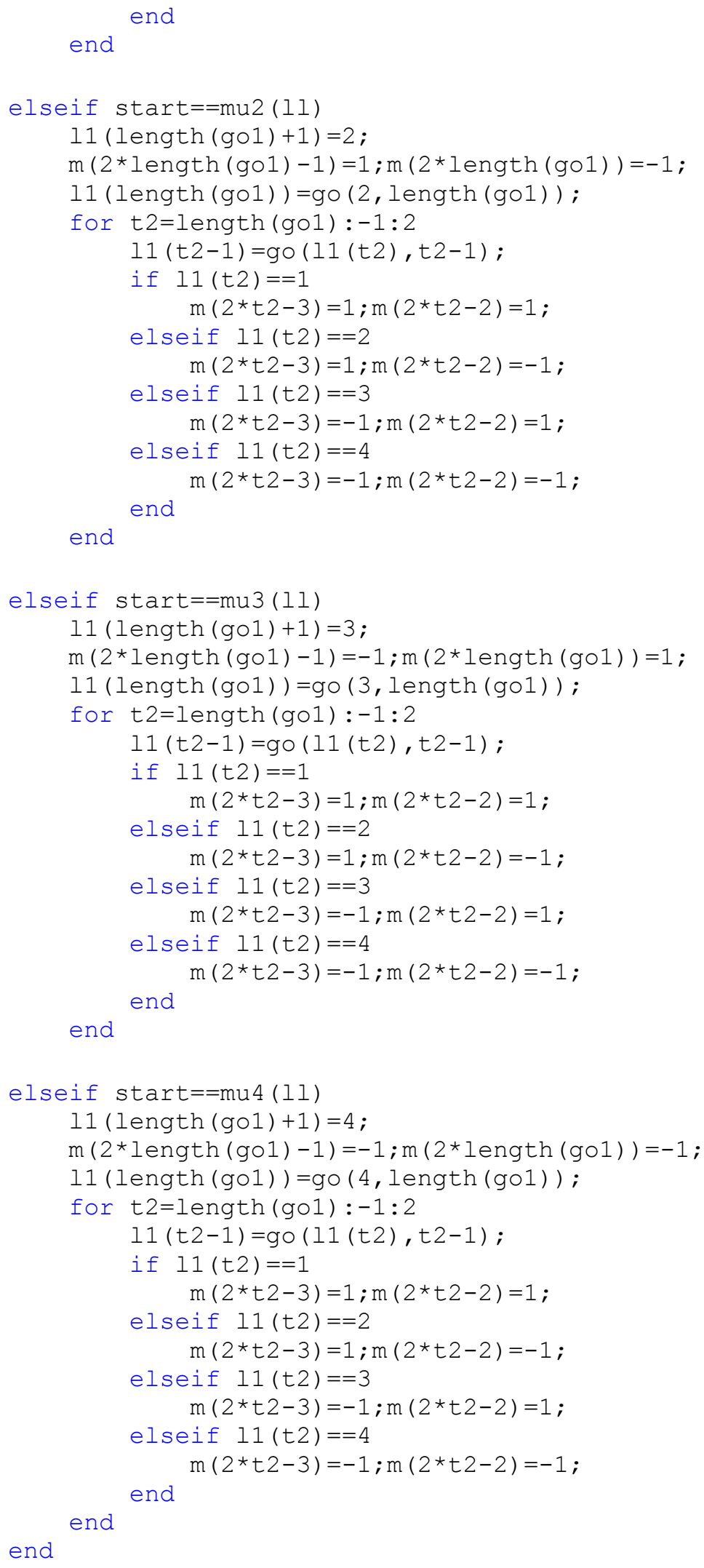




\section{APPENDIX D (Continued)}

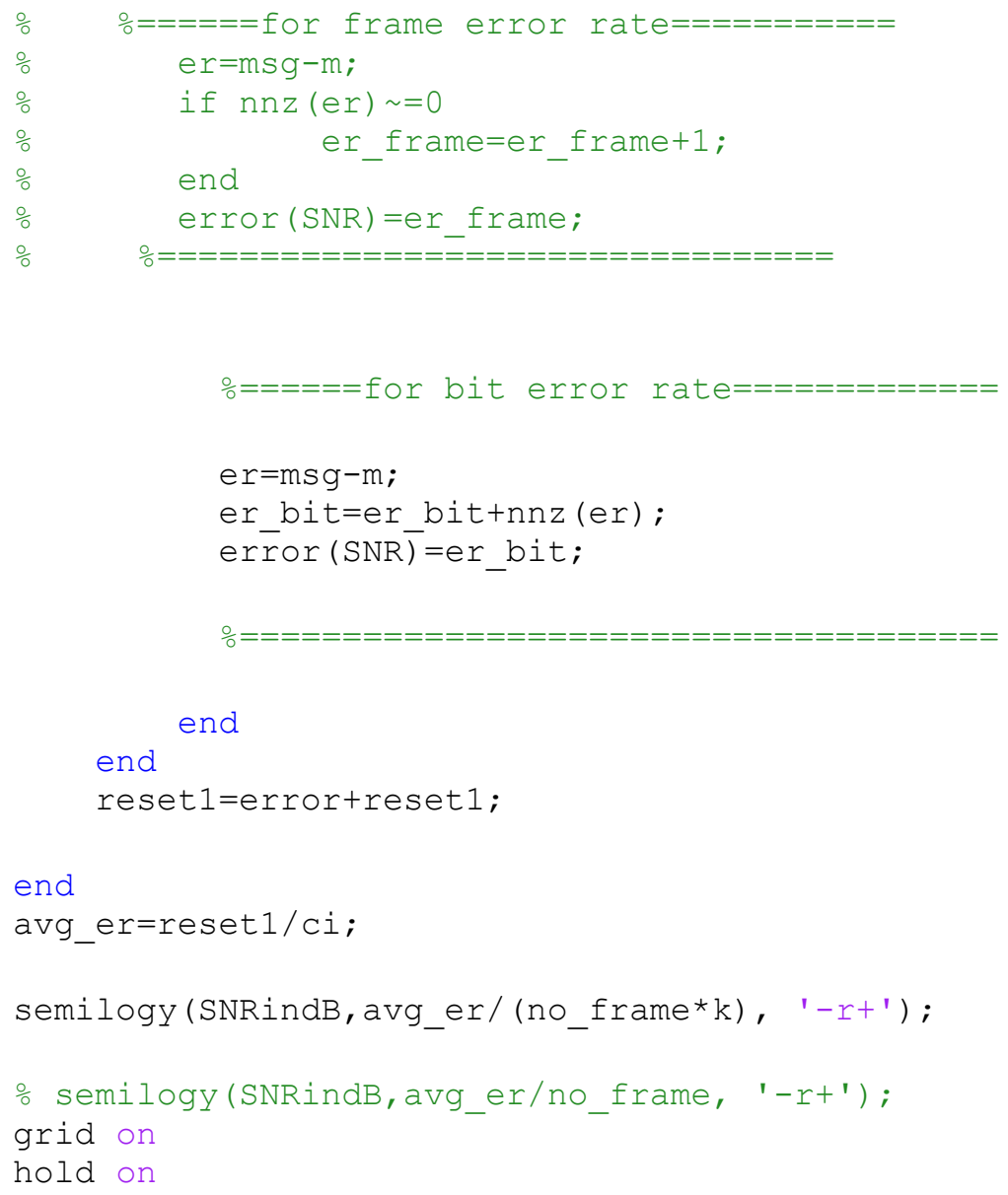




\section{APPENDIX E}

\section{PROGRAM OF DSTBC RELAY COMBINED WITH AF}

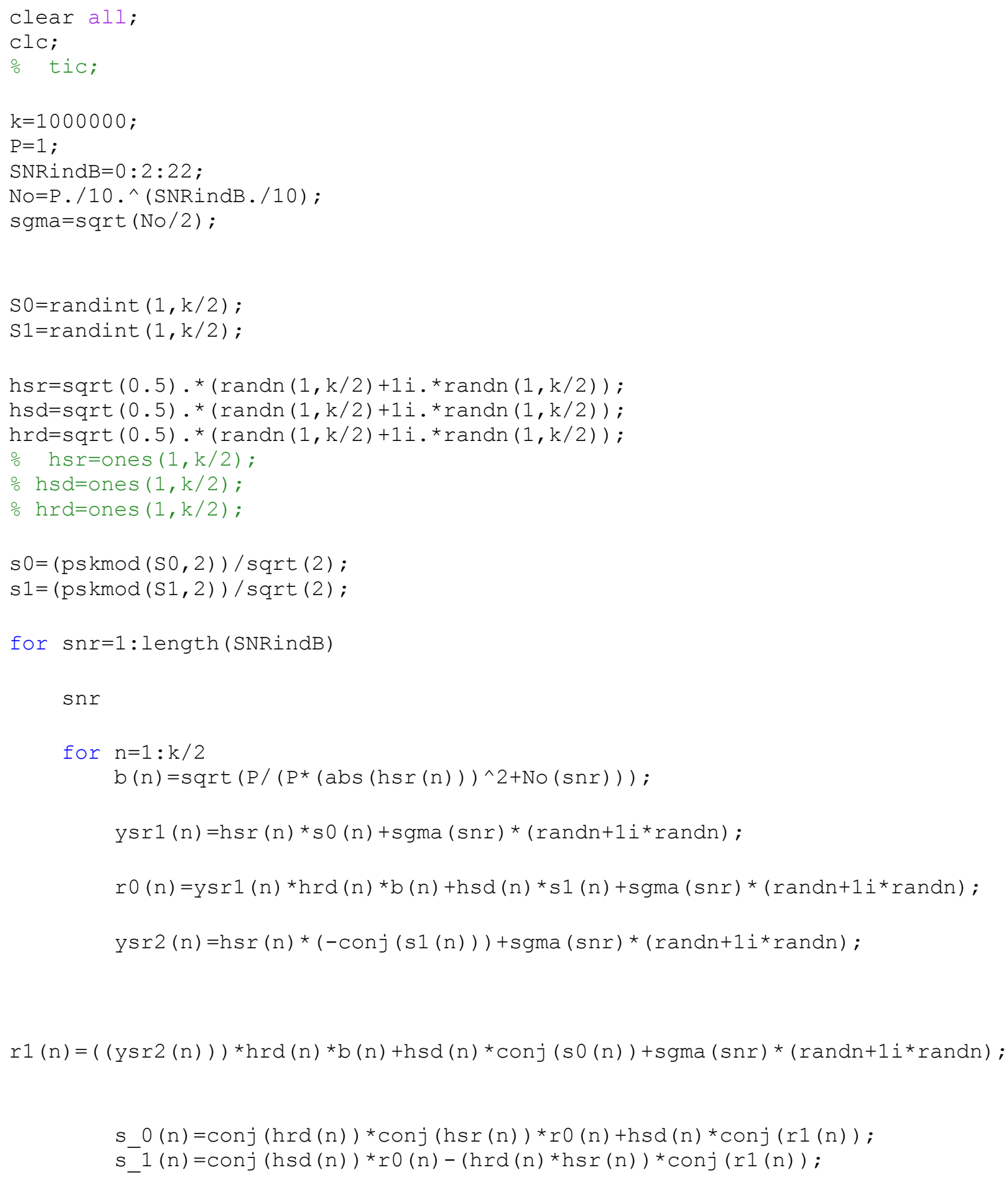




\section{APPENDIX E (Continued)}

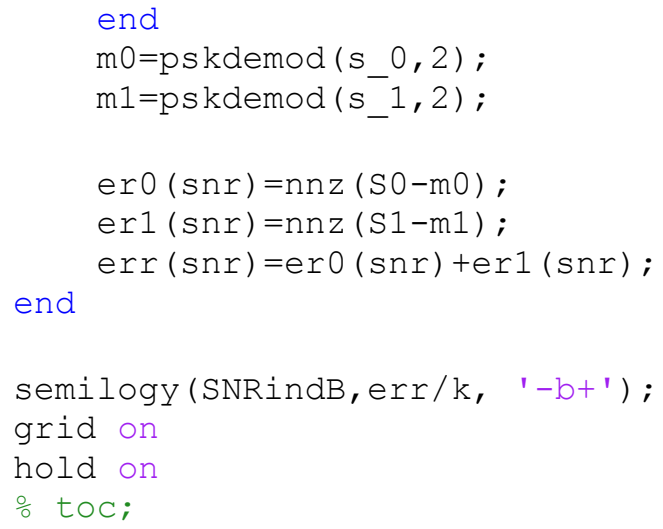




\section{APPENDIX F}

\section{PROGRAM OF ZHANG'S CODE IN CASE OF JAKES' FADING}

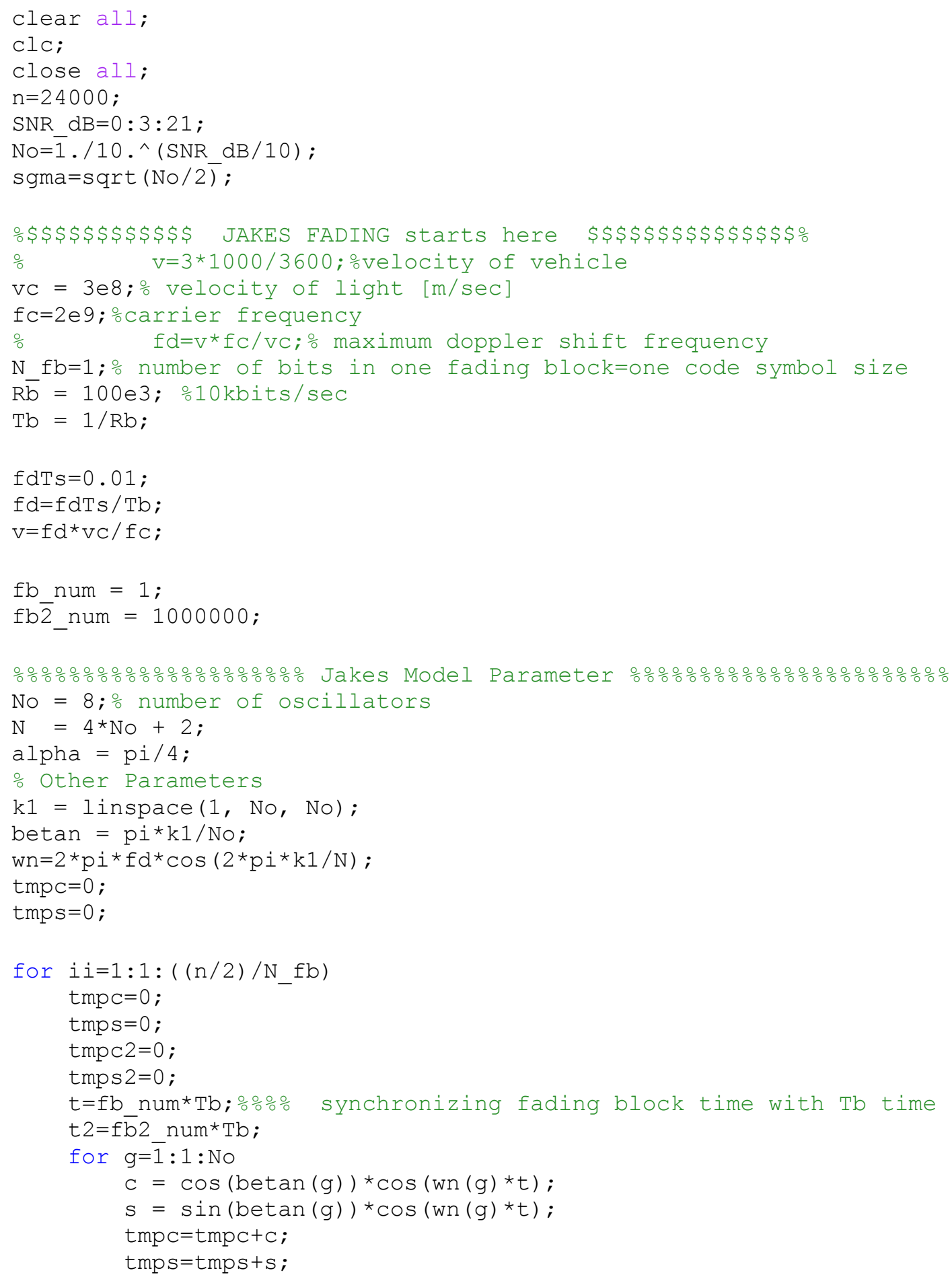




\section{APPENDIX F (Continued)}

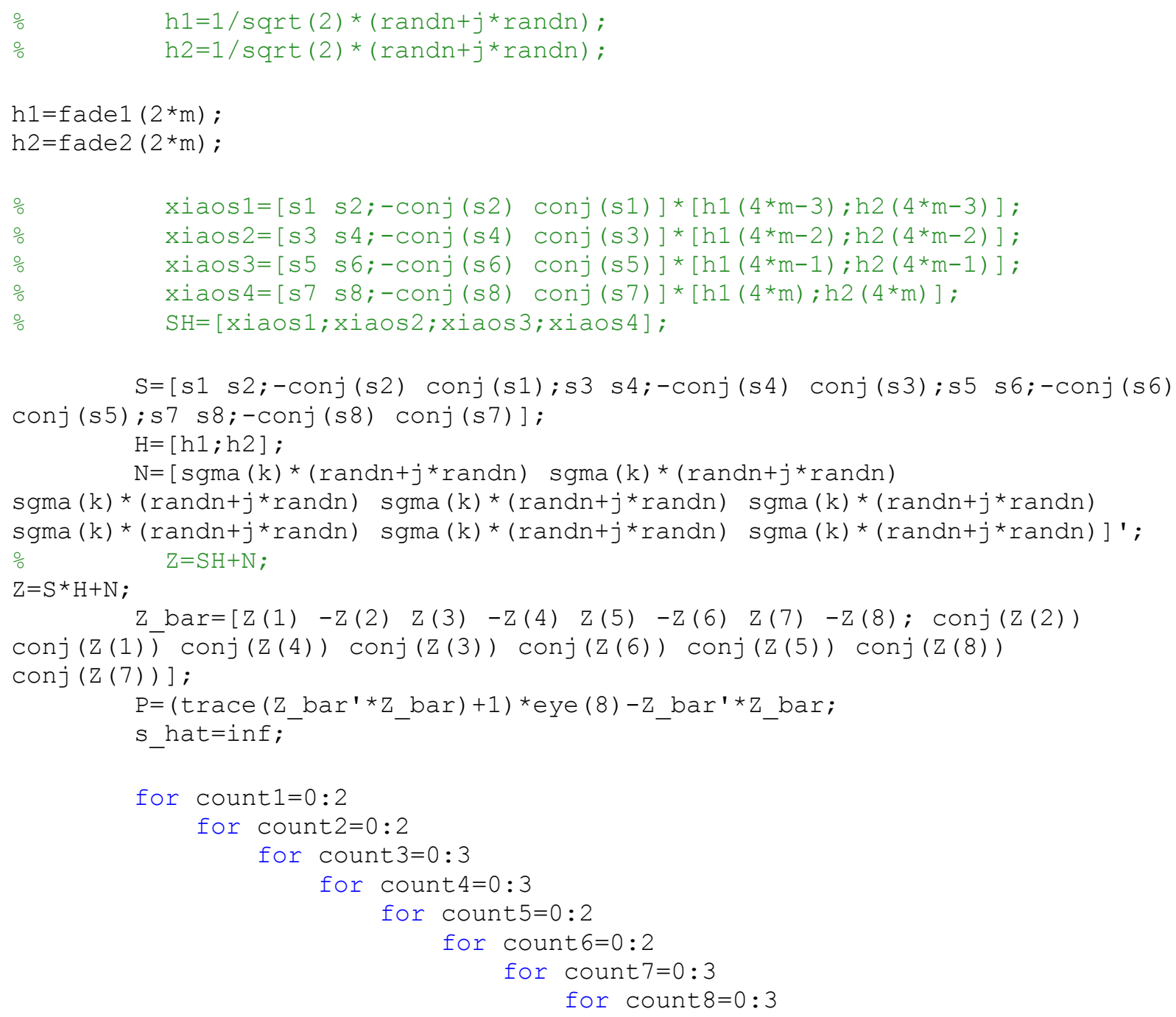




\section{APPENDIX F (Continued)}

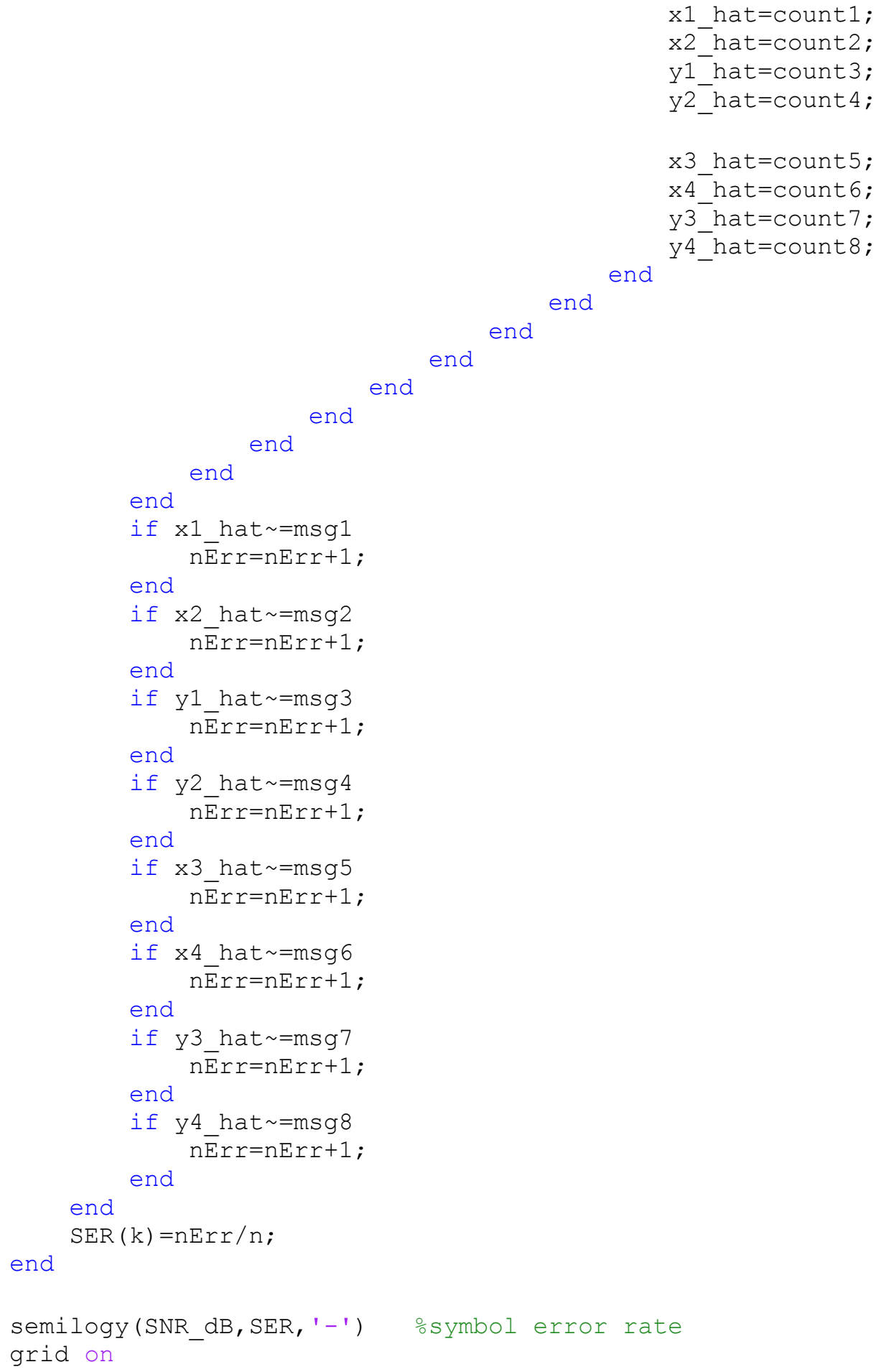




\section{APPENDIX G}

\section{PROGRAM OF QPSK BLIND VITERBI IN CASE OF JAKES' FADING}

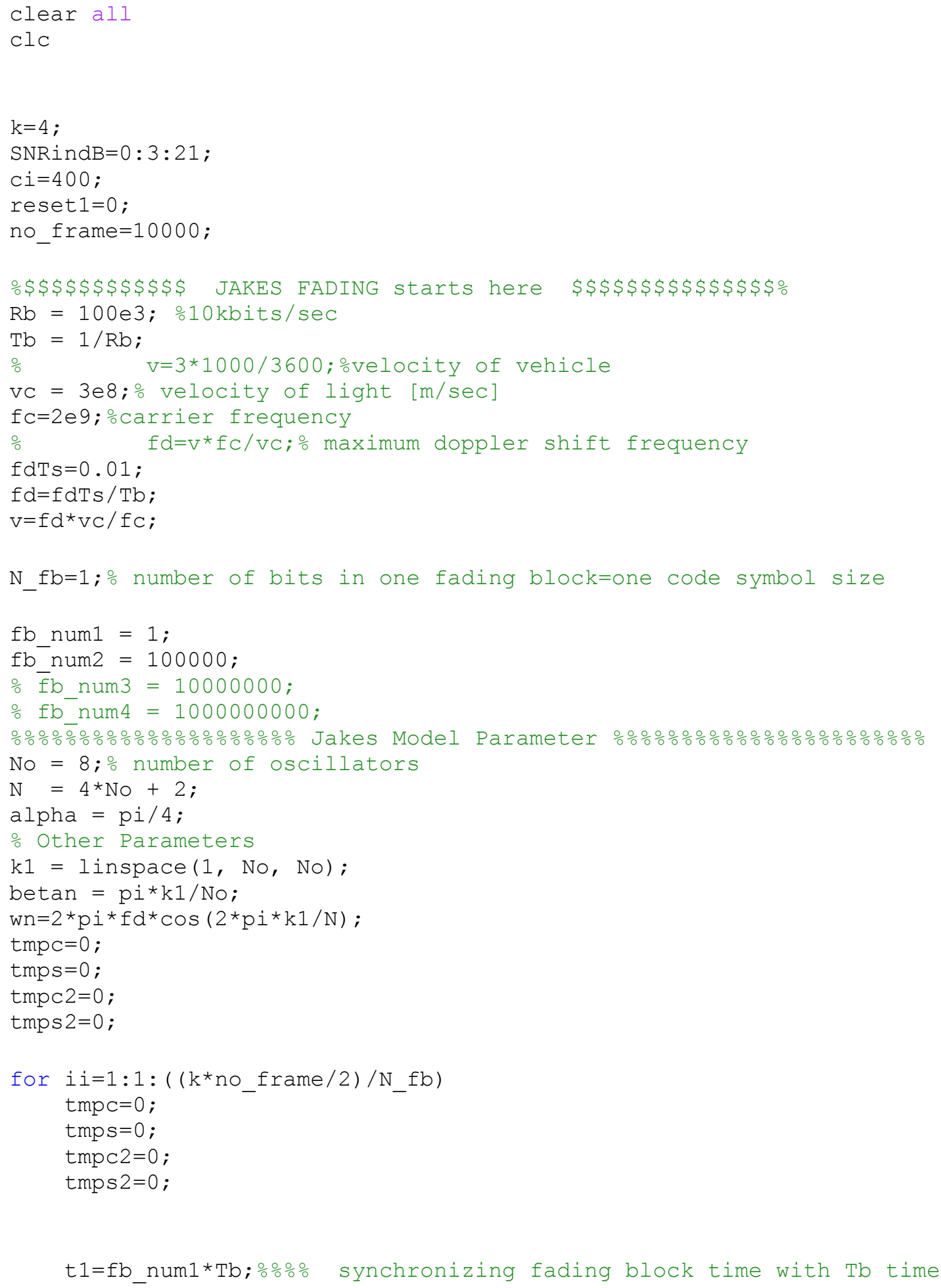




\section{APPENDIX G (Continued)}

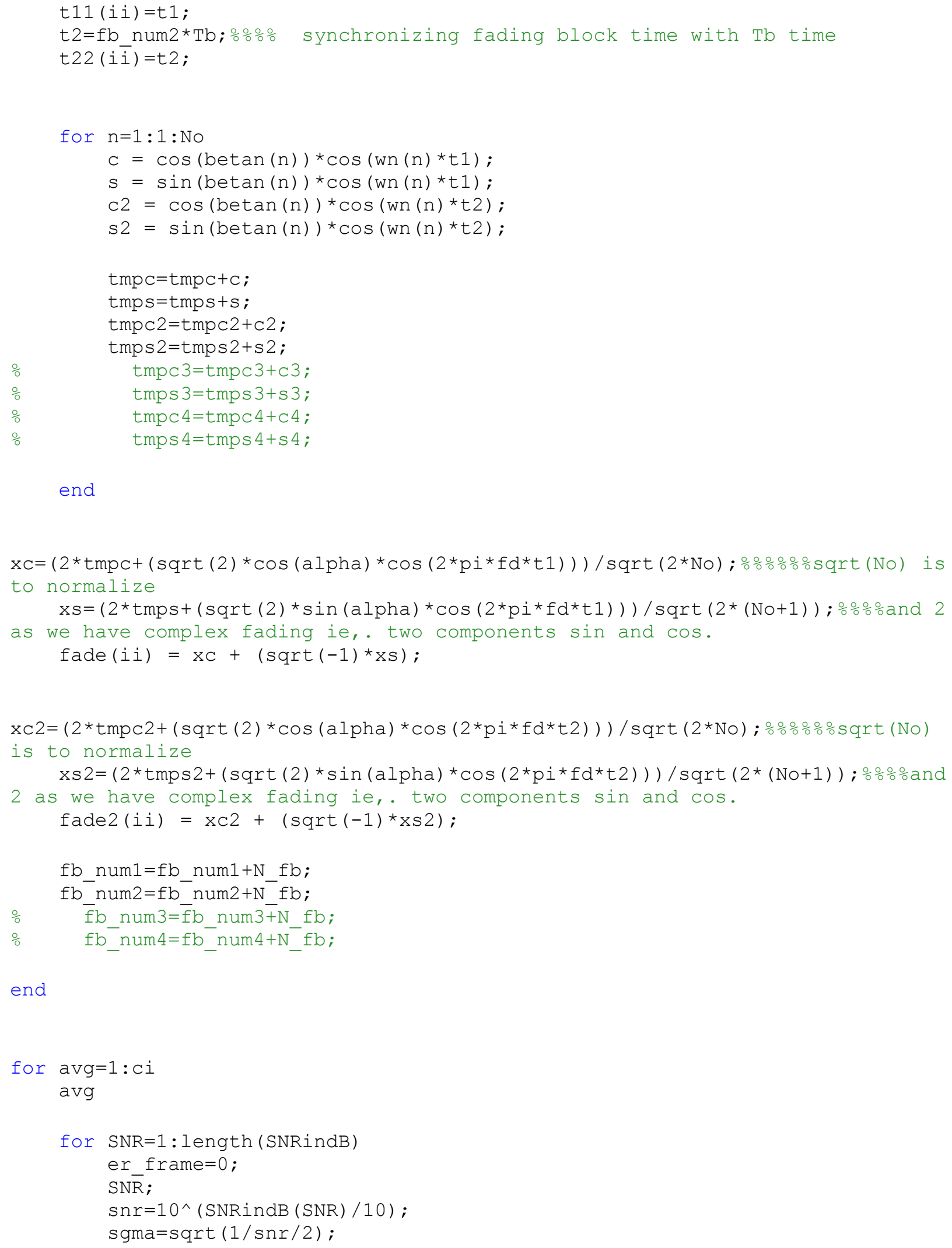




\section{APPENDIX G (Continued)}

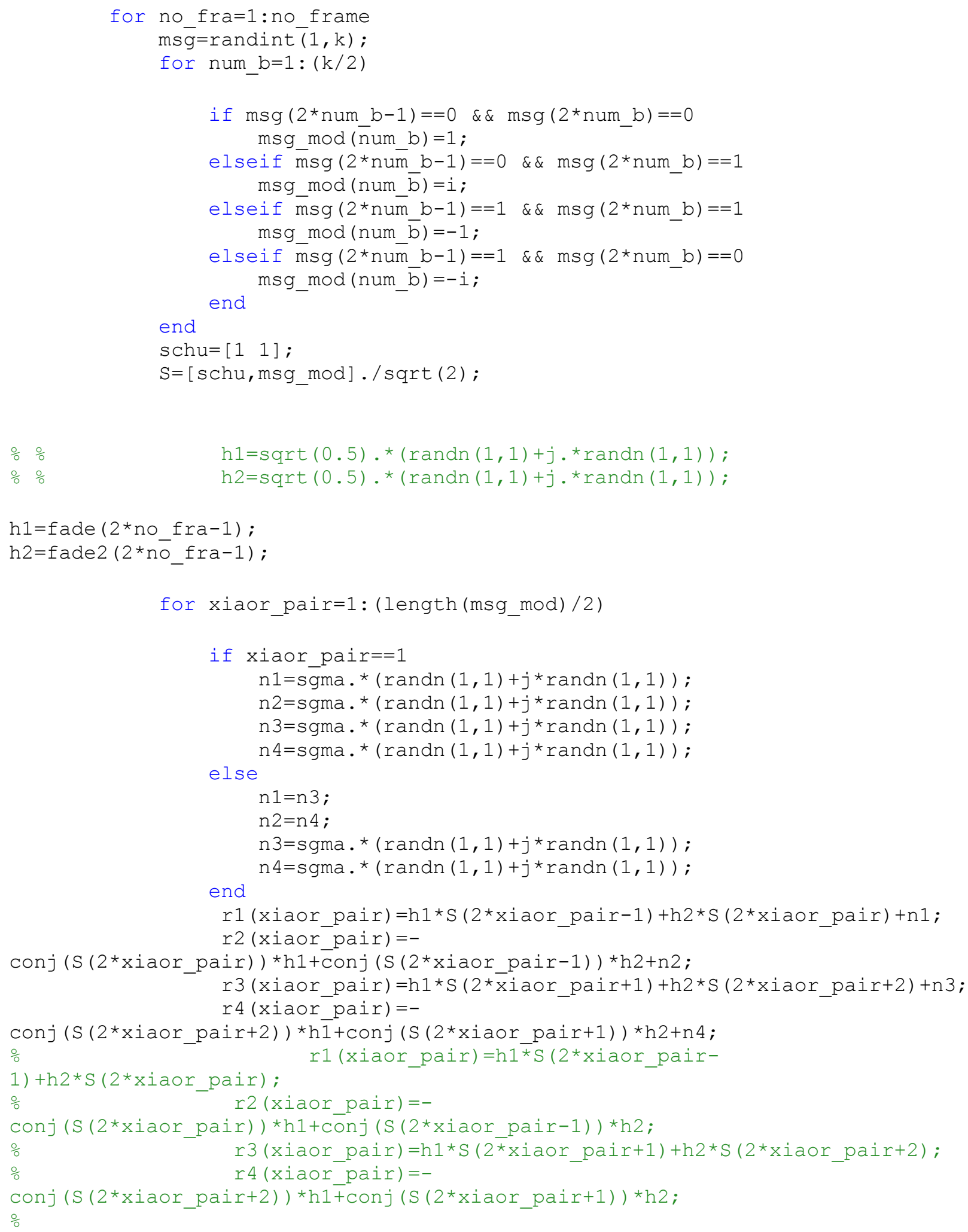




\section{APPENDIX G (Continued)}

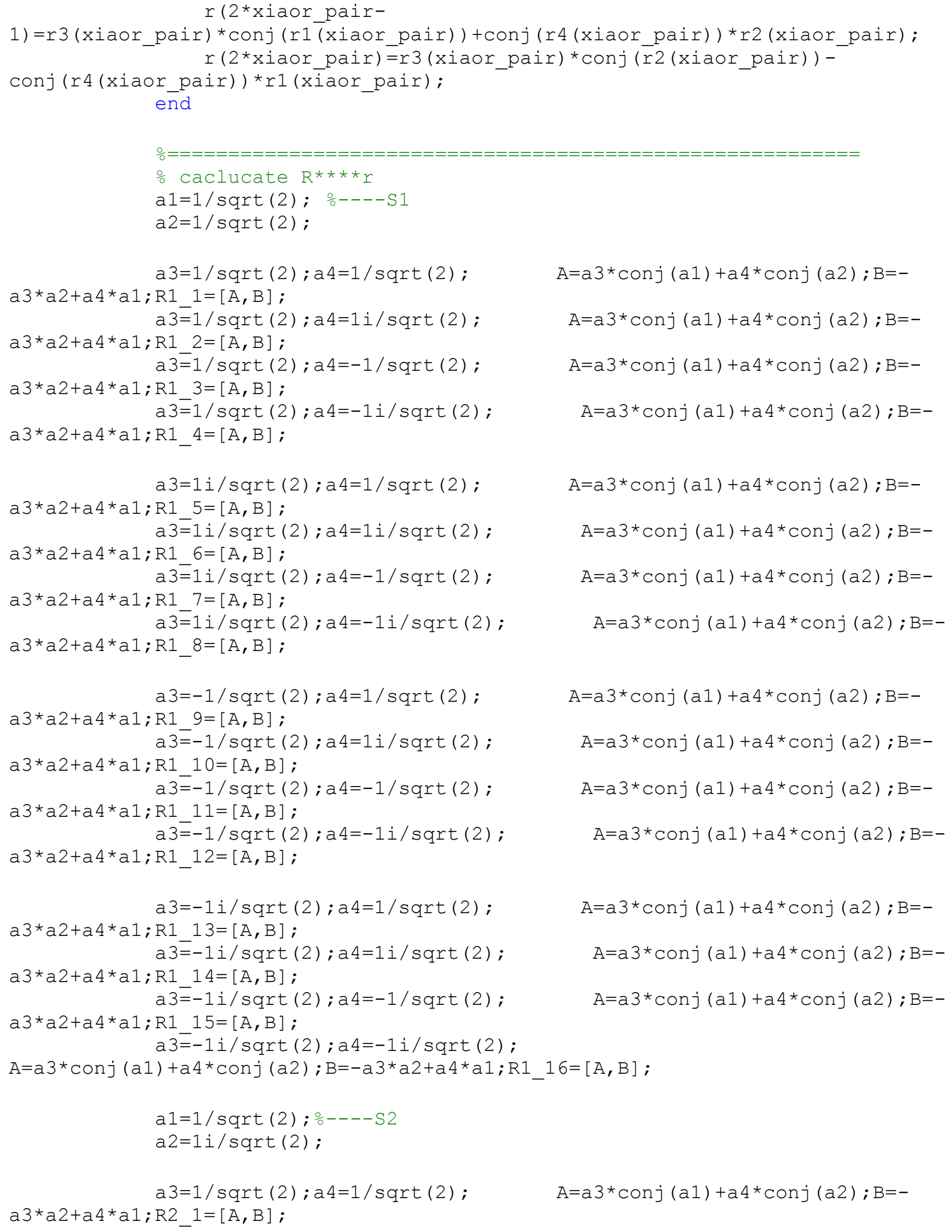




\section{APPENDIX G (Continued)}

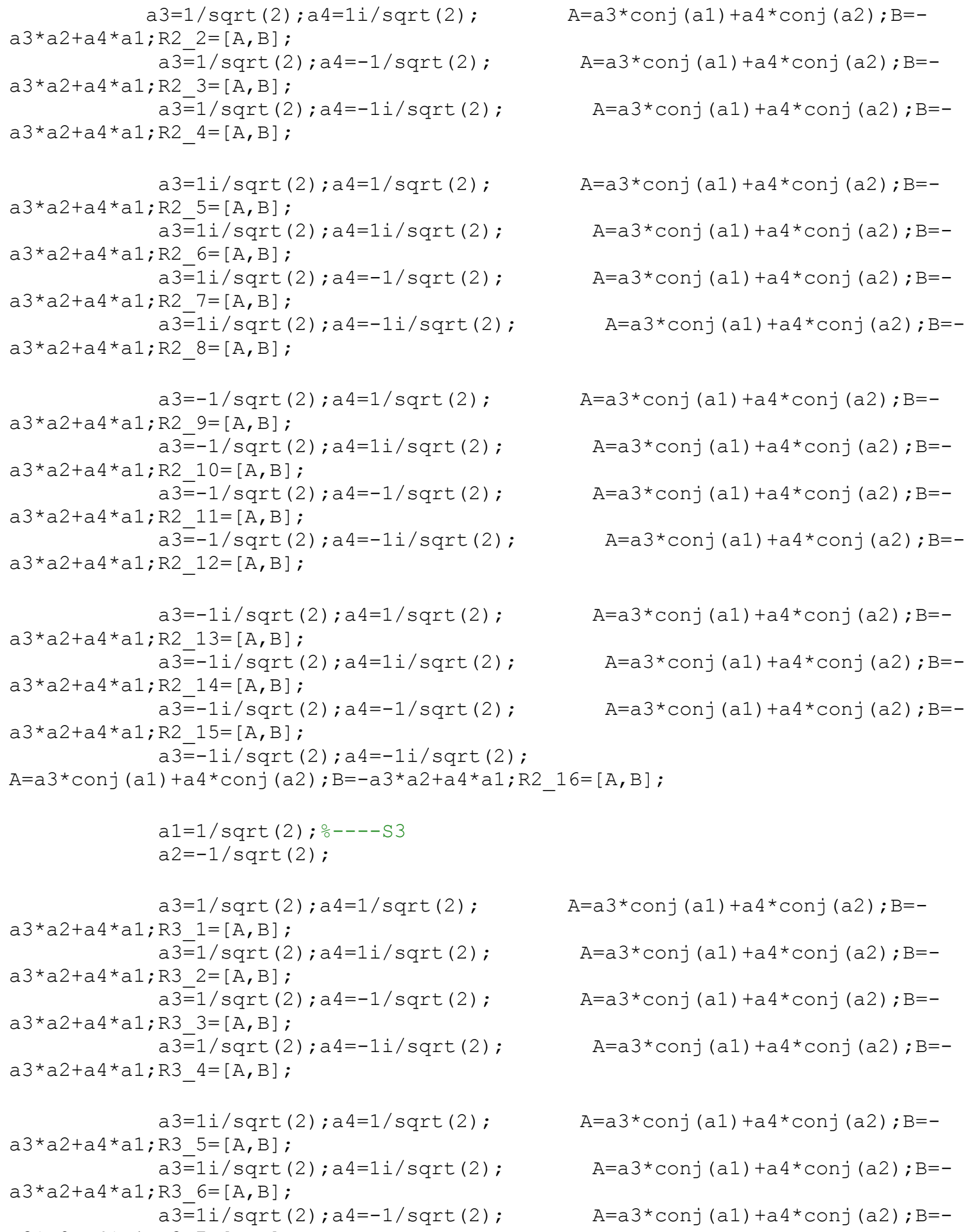




\section{APPENDIX G (Continued)}

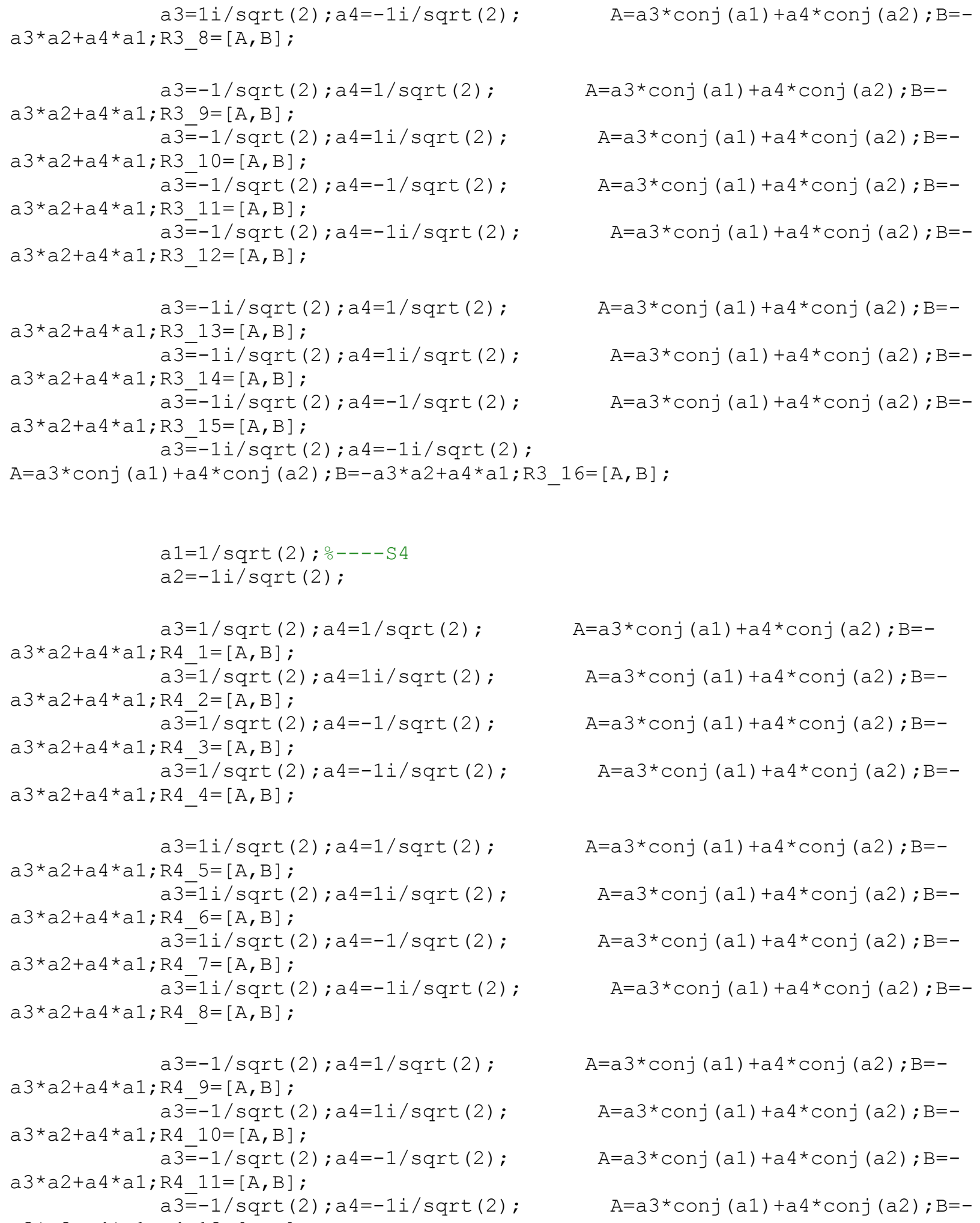




\section{APPENDIX G (Continued)}

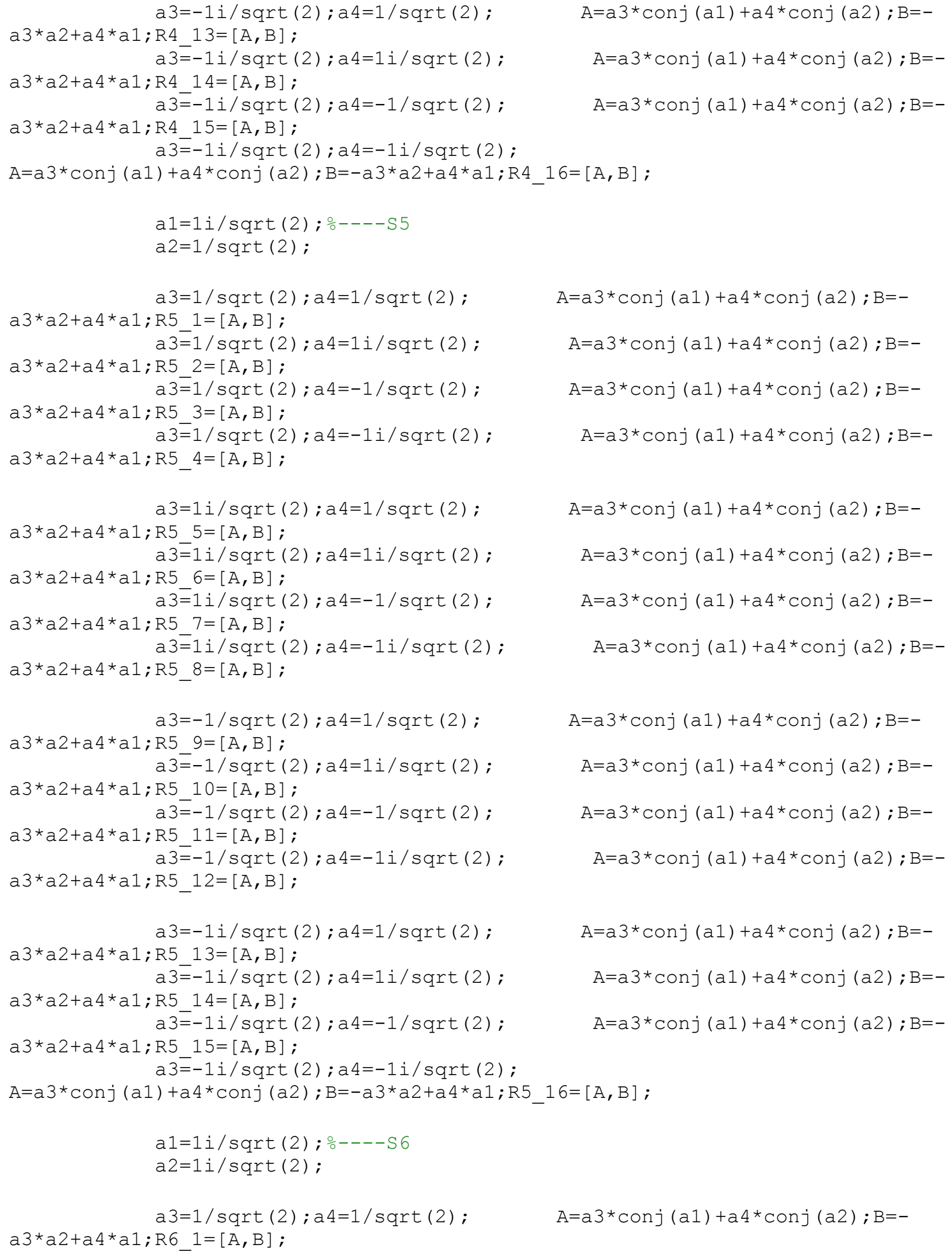




\section{APPENDIX G (Continued)}

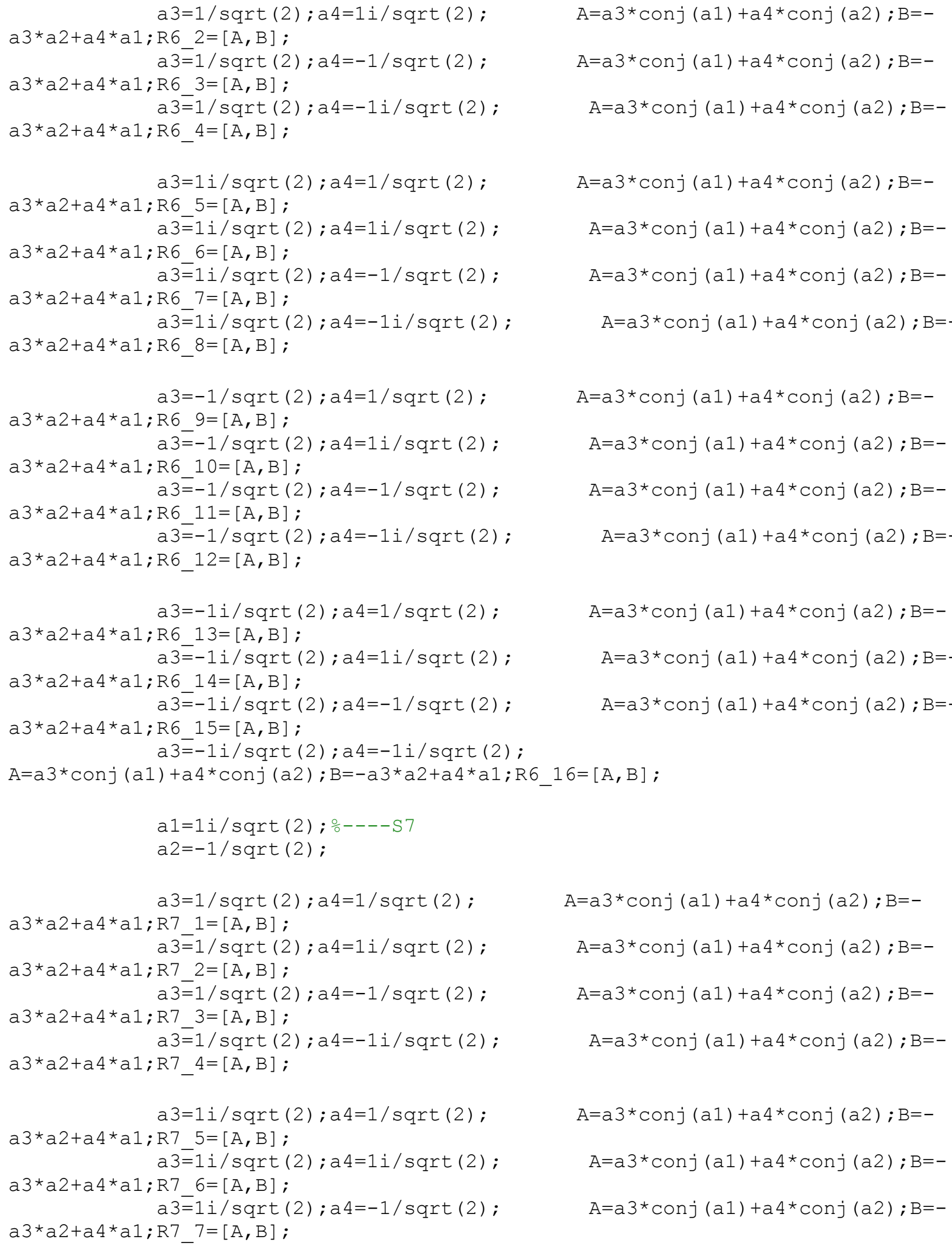




\section{APPENDIX G (Continued)}

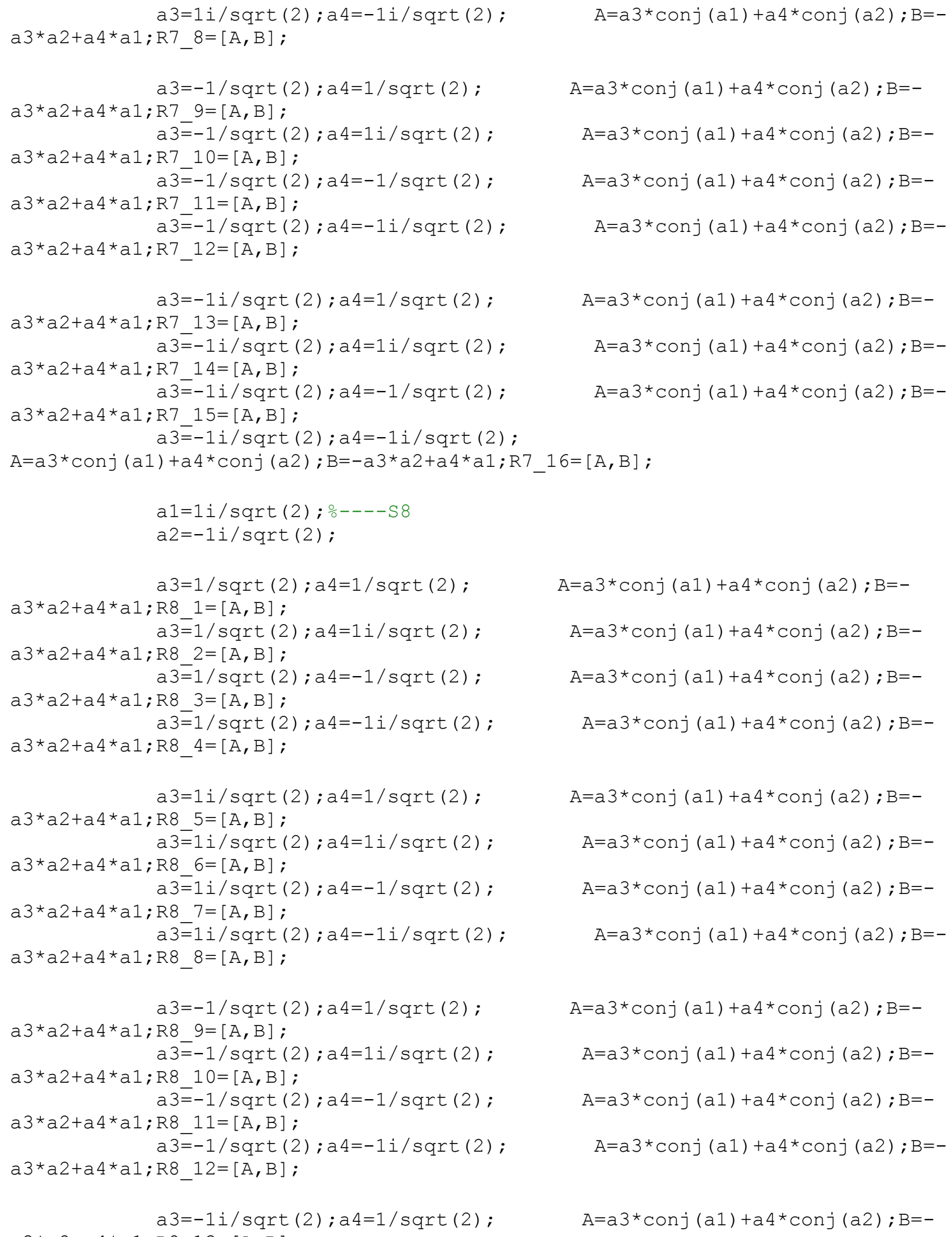




\section{APPENDIX G (Continued)}

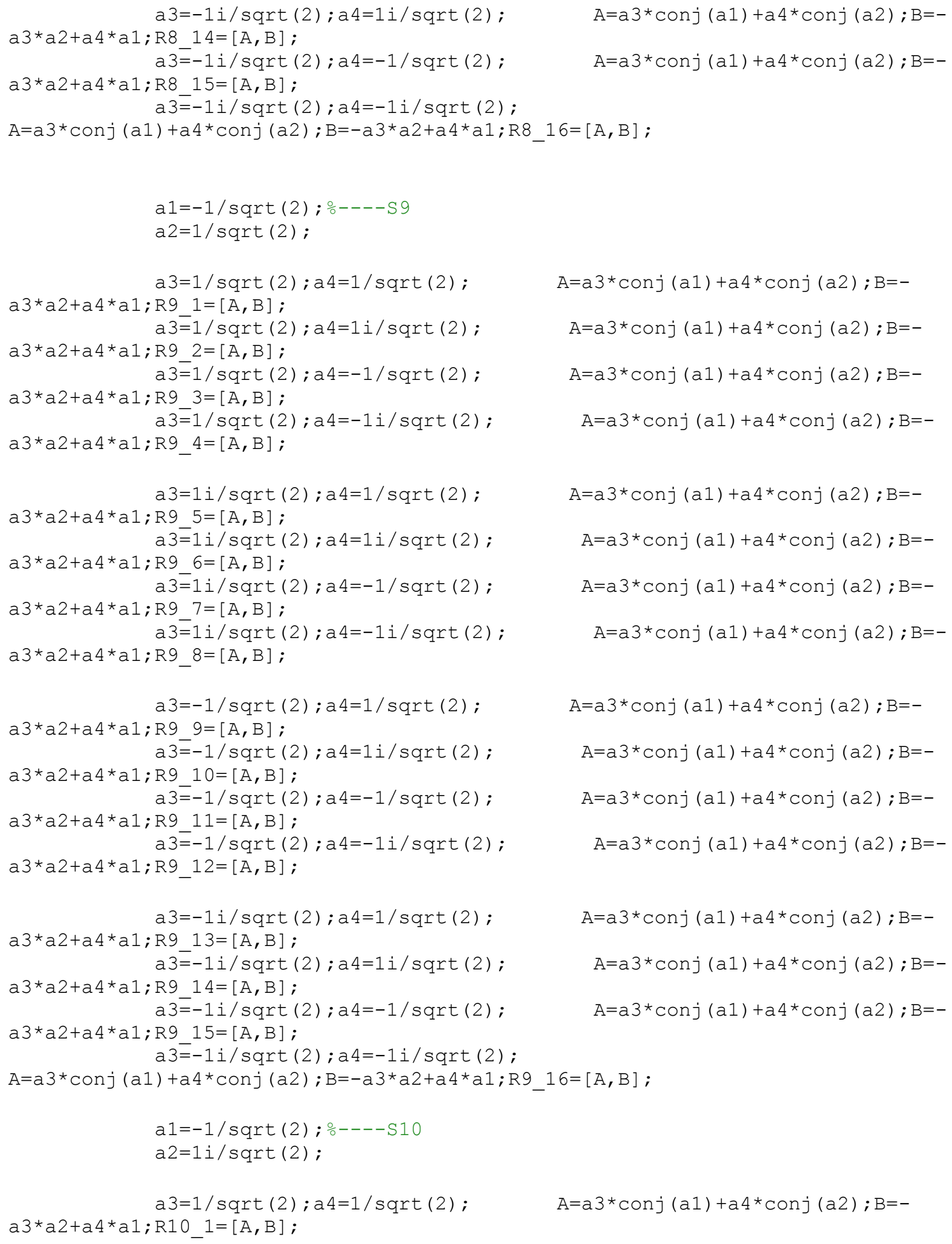




\section{APPENDIX G (Continued)}

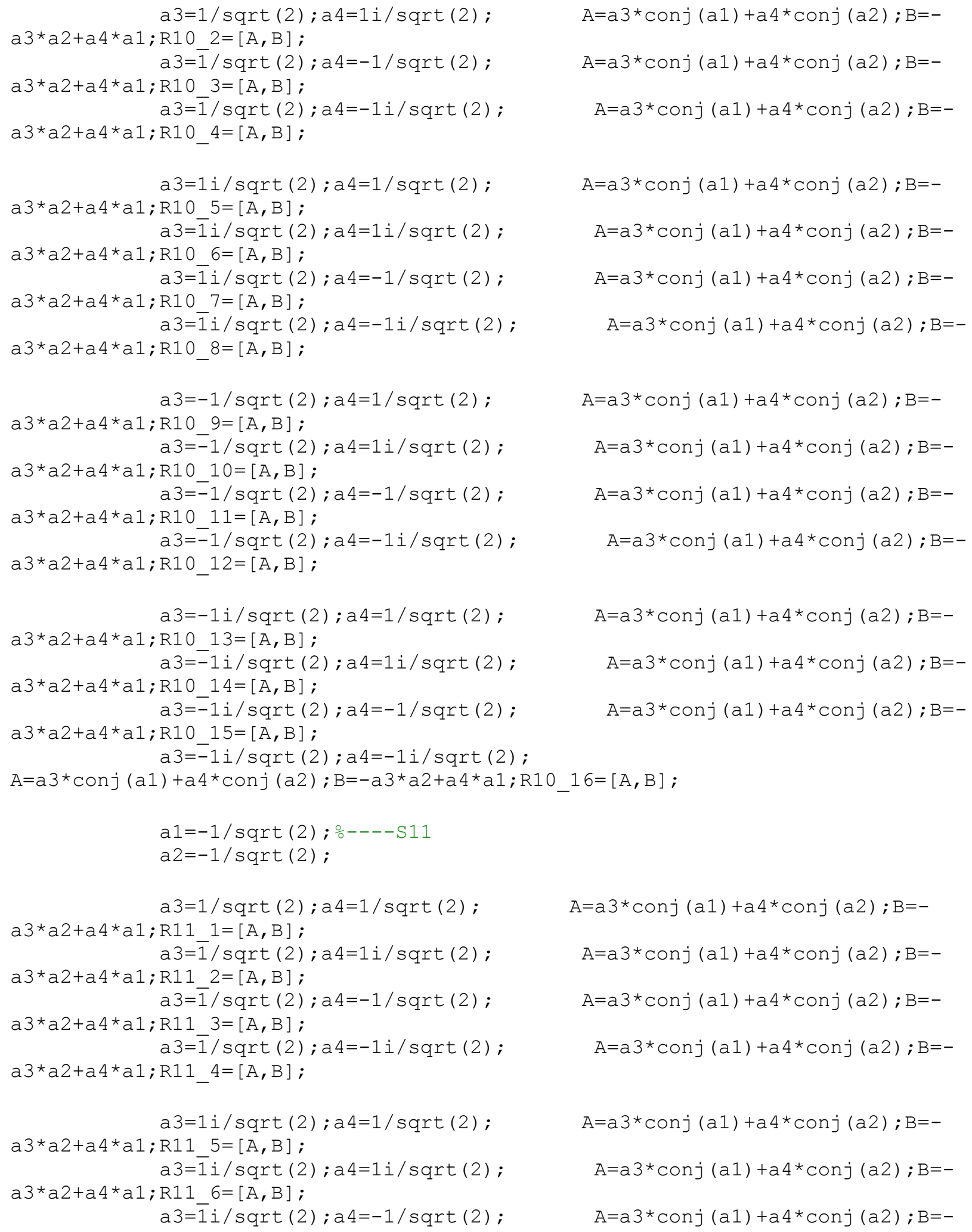




\section{APPENDIX G (Continued)}

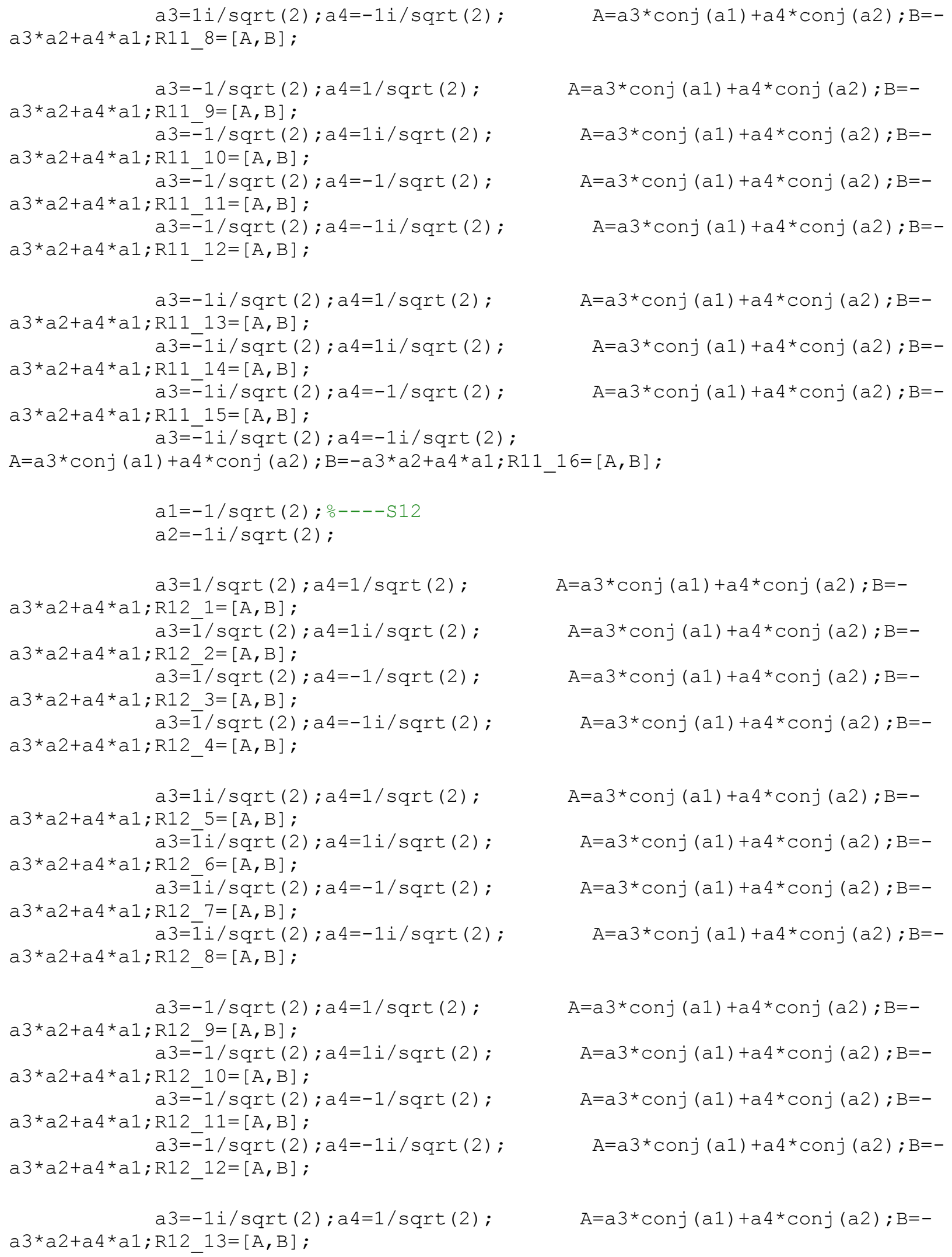




\section{APPENDIX G (Continued)}






\section{APPENDIX G (Continued)}

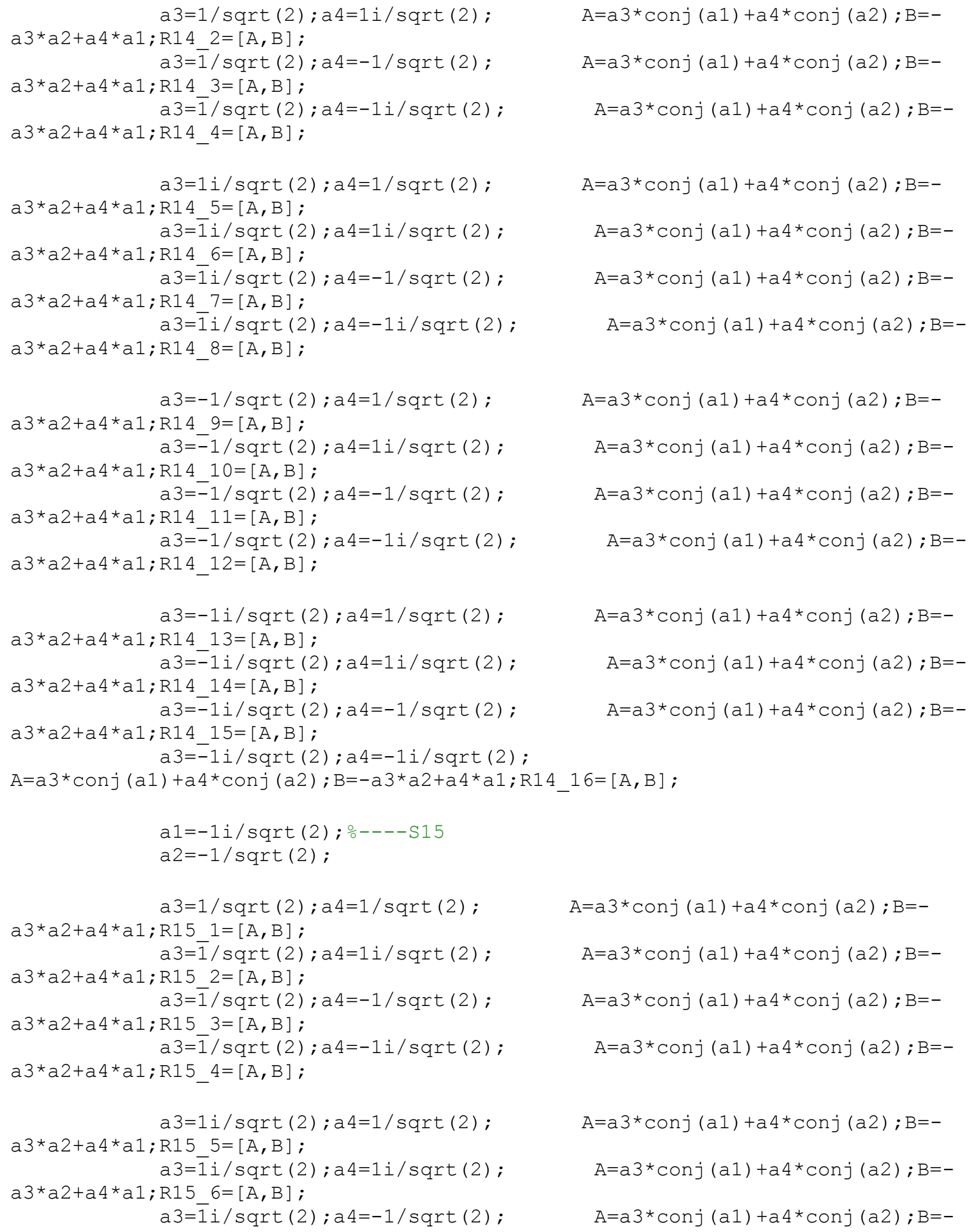




\section{APPENDIX G (Continued)}

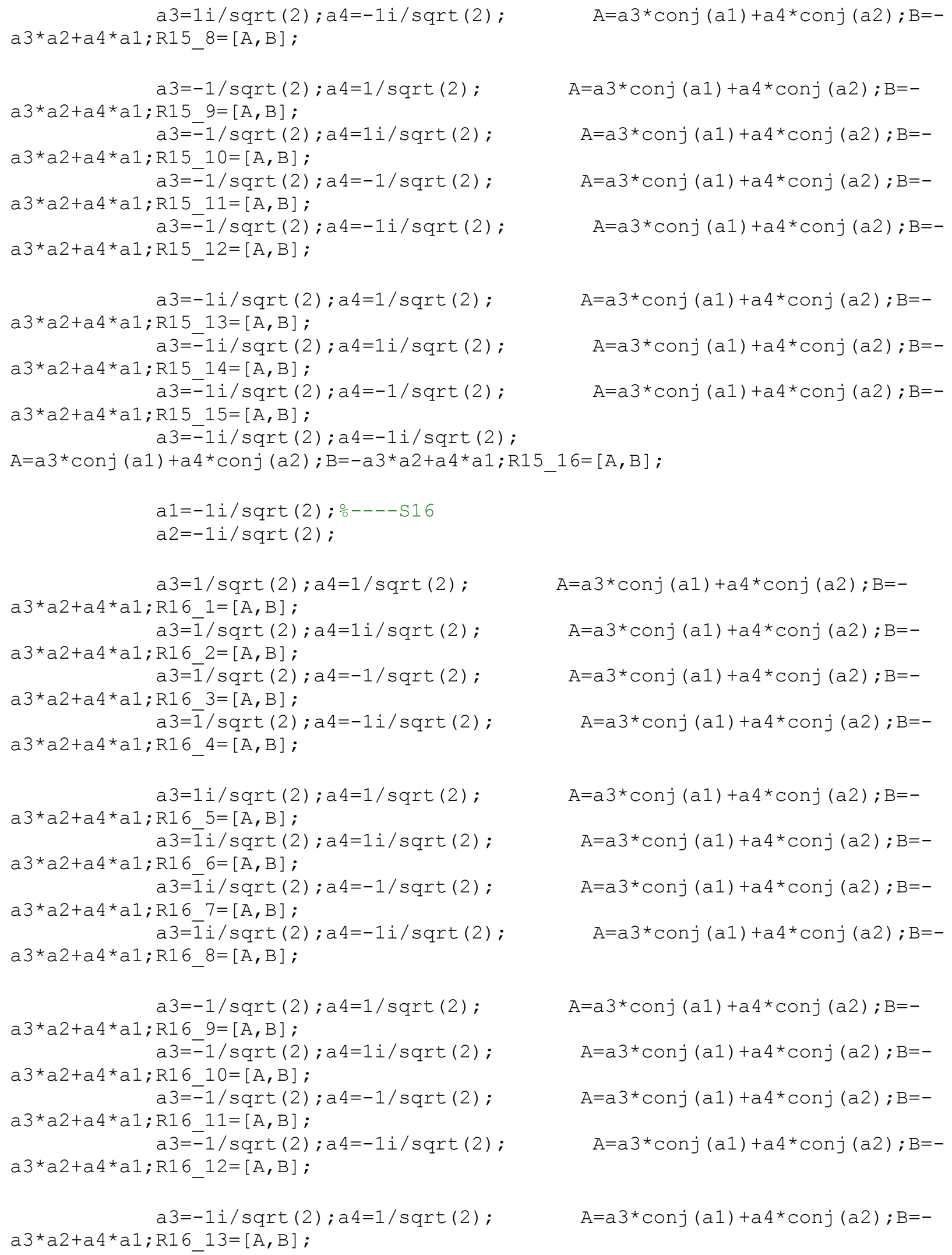




\section{APPENDIX G (Continued)}

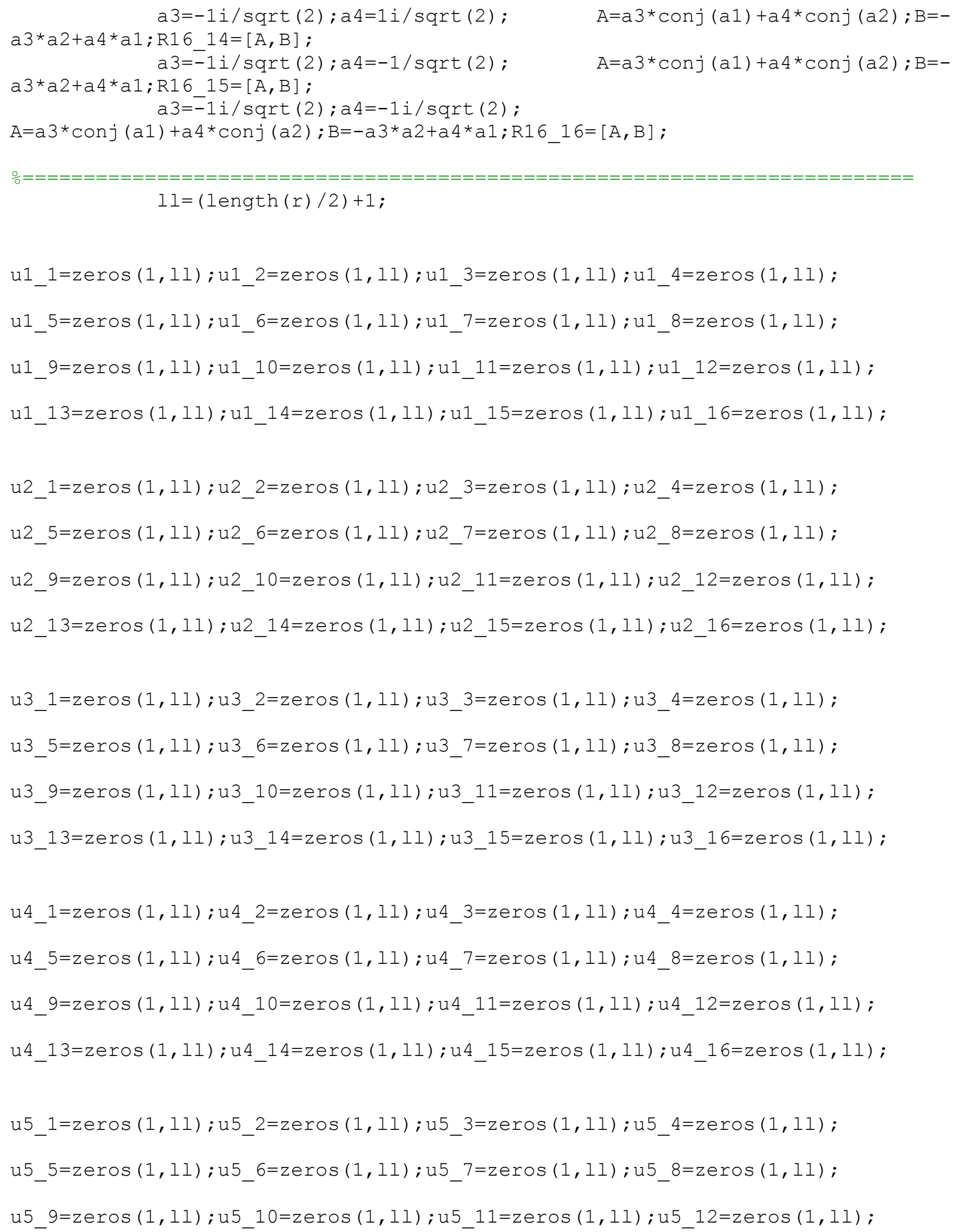




\section{APPENDIX G (Continued)}

u5_13=zeros $(1,11) ; u 5 \_14=\operatorname{zeros}(1,11) ; u 5 \_15=\operatorname{zeros}(1,11) ; u 5 \_16=\operatorname{zeros}(1,11) ;$

u6_1=zeros $(1,11) ; u 6 \_2=\operatorname{zeros}(1,11) ; u 6 \_3=\operatorname{zeros}(1,11) ; u 6 \_4=\operatorname{zeros}(1,11) ;$ u6_5=zeros $(1,11) ; u 6 \_6=\operatorname{zeros}(1,11) ; u 6 \_7=\operatorname{zeros}(1,11) ; u 6 \_8=\operatorname{zeros}(1,11) ;$ u6_9=zeros $(1,11) ; u 6_{1} 10=\operatorname{zeros}(1,11) ; u 6_{-} 11=\operatorname{zeros}(1,11) ; u 6 \_12=\operatorname{zeros}(1,11)$; u6_13=zeros $(1,11) ; u 6 \_14=\operatorname{zeros}(1,11) ; u 6 \_15=\operatorname{zeros}(1,11) ; u 6 \_16=\operatorname{zeros}(1,11) ;$ u7_1=zeros $(1,11) ; u 7 \_2=\operatorname{zeros}(1,11) ; u 7 \_3=\operatorname{zeros}(1,11) ; u 7 \_4=\operatorname{zeros}(1,11)$;

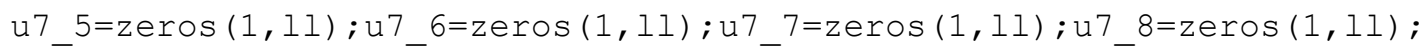
u7_9=zeros $(1,11) ; u 7 \_10=\operatorname{zeros}(1,11) ; u 7 \_11=\operatorname{zeros}(1,11) ; u 7 \_12=\operatorname{zeros}(1,11)$; u7_13=zeros $(1,11) ; u 7 \_14=\operatorname{zeros}(1,11) ; u 7 \_15=\operatorname{zeros}(1,11) ; u 7 \_16=\operatorname{zeros}(1,11) ;$

u8_1=zeros $(1,11) ; u 8 \_2=\operatorname{zeros}(1,11) ; u 8 \_3=\operatorname{zeros}(1,11) ; u 8 \_4=\operatorname{zeros}(1,11)$; u8_5=zeros $(1,11) ; u 8 \_6=\operatorname{zeros}(1,11) ; u 8_{-} 7=\operatorname{zeros}(1,11) ; u 8 \_8=\operatorname{zeros}(1,11)$; u8_9=zeros $(1,11) ; u 8 \_10=\operatorname{zeros}(1,11) ; u 8 \_11=\operatorname{zeros}(1,11) ; u 8 \_12=\operatorname{zeros}(1,11)$; u8_13=zeros $(1,11) ; u 8 \_14=\operatorname{zeros}(1,11) ; u 8 \_15=\operatorname{zeros}(1,11) ; u 8 \_16=\operatorname{zeros}(1,11) ;$

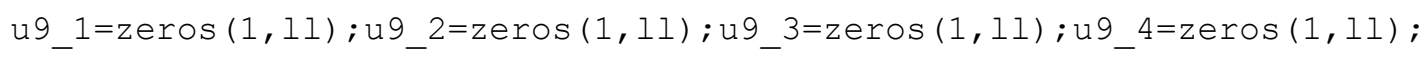
u9_5=zeros $(1,11) ; u 9 \_6=\operatorname{zeros}(1,11) ; u 9 \_7=\operatorname{zeros}(1,11) ; u 9 \_8=\operatorname{zeros}(1,11)$; u9_9=zeros $(1,11) ; u 9 \_10=\operatorname{zeros}(1,11) ; u 9 \_11=\operatorname{zeros}(1,11) ;$ u9_12=zeros $(1,11)$; u9_13=zeros $(1,11) ; u 9 \_14=\operatorname{zeros}(1,11) ; u 9 \_15=\operatorname{zeros}(1,11) ; u 9 \_16=\operatorname{zeros}(1,11)$; u10_1=zeros $(1,11) ; u 10 \_2=\operatorname{zeros}(1,11) ; u 10 \_3=\operatorname{zeros}(1,11) ; u 10 \_4=\operatorname{zeros}(1,11) ;$ u10_5=zeros $(1,11) ; u 10_{-} 6=\operatorname{zeros}(1,11) ; u 10_{-} 7=\operatorname{zeros}(1,11) ; u 10_{-} 8=\operatorname{zeros}(1,11) ;$ u10_9=zeros $(1,11) ; u 10 \_10=\operatorname{zeros}(1,11) ; u 10 \_11=\operatorname{zeros}(1,11) ; u 10 \_12=\operatorname{zeros}(1,11) ;$ u10_13=zeros $(1,11) ; u 10 \_14=\operatorname{zeros}(1,11) ; u 10 \_15=\operatorname{zeros}(1,11) ; u 10 \_16=\operatorname{zeros}(1,11)$;

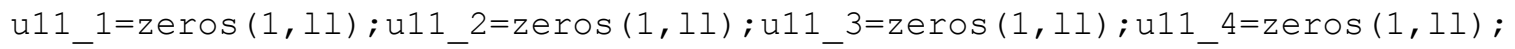
u11_5=zeros $(1,11) ; u 11_{-} 6=\operatorname{zeros}(1,11) ; u 11_{-} 7=\operatorname{zeros}(1,11) ; u 11_{-} 8=\operatorname{zeros}(1,11) ;$ 


\section{APPENDIX G (Continued)}

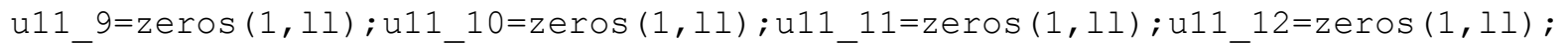

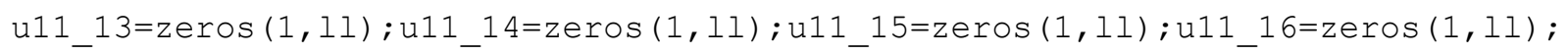

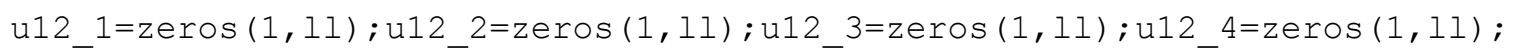

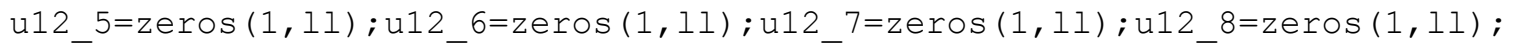
u12_9=zeros $(1,11) ; u 12 \_10=\operatorname{zeros}(1,11) ; u 12 \_11=\operatorname{zeros}(1,11) ; u 12 \_12=\operatorname{zeros}(1,11)$; u12_13=zeros $(1,11) ; u 12 \_14=\operatorname{zeros}(1,11) ; u 12 \_15=\operatorname{zeros}(1,11) ; u 12 \_16=\operatorname{zeros}(1,11)$;

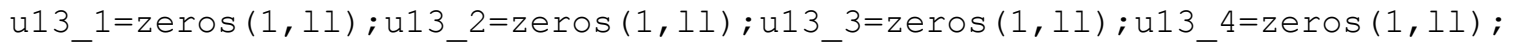

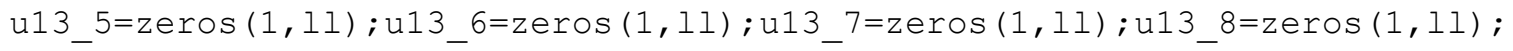
u13_9=zeros $(1,11) ; u 13 \_10=\operatorname{zeros}(1,11) ; u 13 \_11=\operatorname{zeros}(1,11) ; u 13 \_12=\operatorname{zeros}(1,11)$; u13_13=zeros $(1,11) ;$ u13_14=zeros $(1,11) ; u 13 \_15=\operatorname{zeros}(1,11) ; u 13 \_16=\operatorname{zeros}(1,11)$;

u14_1=zeros $(1,11) ; u 14 \_2=\operatorname{zeros}(1,11) ; u 14 \_3=\operatorname{zeros}(1,11) ; u 14 \_4=\operatorname{zeros}(1,11)$;

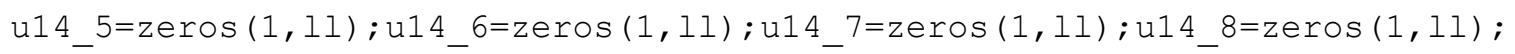
u14_9=zeros $(1,11) ; u 14 \_10=\operatorname{zeros}(1,11) ; u 14 \_11=\operatorname{zeros}(1,11) ; u 14 \_12=\operatorname{zeros}(1,11)$;

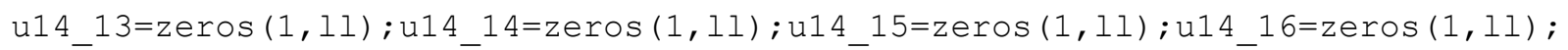

u15_1=zeros $(1,11) ;$ u15_2=zeros $(1,11) ; u 15 \_3=\operatorname{zeros}(1,11) ; u 15 \_4=\operatorname{zeros}(1,11)$; u15_5=zeros $(1,11) ;$ u15_6=zeros $(1,11) ; u 15 \_7=\operatorname{zeros}(1,11) ; u 15$ 8=zeros $(1,11)$; u15_9=zeros $(1,11) ; u 15 \_10=\operatorname{zeros}(1,11) ; u 15 \_11=\operatorname{zeros}(1,11) ; u 15 \_12=\operatorname{zeros}(1,11)$; u15_13=zeros $(1,11) ; u 15 \_14=\operatorname{zeros}(1,11) ; u 15 \_15=\operatorname{zeros}(1,11) ; u 15 \_16=\operatorname{zeros}(1,11)$;

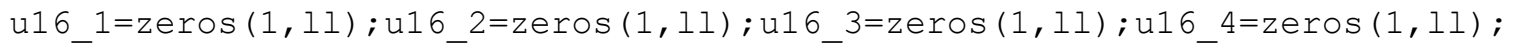
u16_5=zeros $(1,11) ; u 16 \_6=\operatorname{zeros}(1,11) ; u 16 \_7=\operatorname{zeros}(1,11) ; u 16 \_8=\operatorname{zeros}(1,11)$; u16_9=zeros $(1,11) ; u 16 \_10=\operatorname{zeros}(1,11) ; u 16 \_11=\operatorname{zeros}(1,11) ; u 16 \_12=\operatorname{zeros}(1,11)$; u16_13=zeros $(1,11) ; u 16 \_14=\operatorname{zeros}(1,11) ; u 16 \_15=\operatorname{zeros}(1,11) ; u 16 \_16=\operatorname{zeros}(1,11)$; $\mathrm{Q}=\operatorname{zeros}(16,11) ; \mathrm{Q}(1,1)=1$; mul=zeros $(1,11) ; \operatorname{mu} 2=\operatorname{zeros}(1,11) ; \operatorname{mu} 3=\operatorname{zeros}(1,11) ; \operatorname{mu} 4=\operatorname{zeros}(1,11)$; mu5=zeros $(1,11) ; \operatorname{mu} 6=\operatorname{zeros}(1,11) ; \operatorname{mu} 7=\operatorname{zeros}(1,11) ; \operatorname{mu} 8=\operatorname{zeros}(1,11)$; 


\section{APPENDIX G (Continued)}

mu $9=\operatorname{zeros}(1,11) ; \operatorname{mu} 10=\operatorname{zeros}(1,11) ; \operatorname{mu} 11=\operatorname{zeros}(1,11) ; \operatorname{mu} 12=\operatorname{zeros}(1,11) ;$

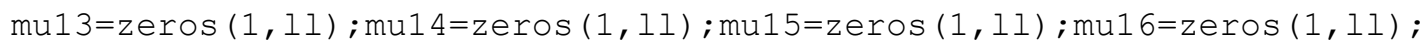

$\div \mathrm{m}=\mathrm{baseQ} 1(r)$

for $t 1=2: 11$

$$
t=t 1-1
$$$$
\text { if } Q(1, t)==1
$$$$
\text { ul_1 } 1(t)=\left(\left(\left(\operatorname{norm}\left(r(2 * t-1)-R_{1} 1(1)\right)\right)^{\wedge} 2\right)+((\operatorname{norm}(r(2 * t)-\right.
$$

$\mathrm{R} 1$ 1 (2)) )^2)); $\mathrm{Q}(1, \mathrm{t} 1) \overline{=} 1$;

R1_2(2)) )^2)); Q (2, t1) $=1$;

$u 12(t 1)=\left(\left(\left(\operatorname{norm}\left(r(2 * t-1)-R 1 \_2(1)\right)\right)^{\wedge} 2\right)+((\operatorname{norm}(r(2 * t)-\right.$

$\left.\left.\left.\left.\mathrm{R} 1 \_3(2)\right)\right)^{\wedge} 2\right)\right) ; \mathrm{Q}(3, \mathrm{t} 1) \overline{=}$;

u1_3(t1) $=\left(\left(\left(\operatorname{norm}\left(r(2 * t-1)-\mathrm{R} 1 \_3(1)\right)\right) \wedge 2\right)+((\operatorname{norm}(r(2 * t)-\right.$

R1_4(2)) )^2)); Q (4,t1) $=1$;

ul_ $4(t 1)=\left(\left(\left(\operatorname{norm}\left(r(2 \star t-1)-R 1_{-} 4(1)\right)\right)^{\wedge} 2\right)+((\operatorname{norm}(r(2 \star t)-\right.$

R1 $\left.\left.5(2)))^{\wedge} 2\right)\right) ; \quad Q(5, t 1)=1 ;$

$\left.\left.\left.\left.\mathrm{R} 1_{-} 6(2)\right)\right)^{\wedge} 2\right)\right) ; \mathrm{Q}(6, \mathrm{t} 1) \overline{=}$;

ul_ $6(t 1)=\left(\left(\left(\operatorname{norm}\left(r(2 * t-1)-\mathrm{R} 1_{-} 6(1)\right)\right)^{\wedge} 2\right)+((\operatorname{norm}(r(2 * t)-\right.$

R1 $\left.\left.7(2)))^{\wedge} 2\right)\right) ; \quad Q(7, t 1)=1 ;$

ul_ $7(t 1)=\left(\left(\left(\operatorname{norm}\left(r(2 \star t-1)-R_{-} 7(1)\right)\right)^{\wedge} 2\right)+((\operatorname{norm}(r(2 \star t)-\right.$

$\left.\left.\left.\left.\mathrm{R} 1_{-} 8(2)\right)\right)^{\wedge} 2\right)\right) ; \mathrm{Q}(8, \mathrm{t} 1) \overline{=}$;

ul $8(t 1)=\left(\left(\left(\operatorname{norm}\left(r(2 * t-1)-\mathrm{R} 1\right.\right.\right.\right.$ s $\left.\left.\left._{1}(1)\right)\right) \wedge 2\right)+((\operatorname{norm}(r(2 * t)-$ ul_ $9(t 1)=\left(\left(\left(\operatorname{norm}\left(r(2 * t-1)-R_{-}{ }^{9}(1)\right)\right)^{\wedge} 2\right)+((\operatorname{norm}(r(2 * t)-\right.$

R1_9(2)) )^2)); $Q(9, t 1) \overline{=}$;

R1_10(2)) )^2)); Q $(10, t 1 \overline{)}=1$;

u1_10 (t1) $=\left(\left(\left(\operatorname{norm}\left(r(2 * t-1)-\mathrm{R}_{-} 10(1)\right)\right)^{\wedge} 2\right)+((\operatorname{norm}(r(2 * t)-\right.$

R1_11(2)) )^2));Q(11,t1) $=1$;

u1 $11(t 1)=\left(\left((\operatorname{norm}(r(2 * t-1)-R 111(1)))^{\wedge} 2\right)+((\operatorname{norm}(r(2 * t)-\right.$

R1_12(2)) )^2));Q(12,t1) $=1$;

1_ $12(t 1)=\left(\left(\left(\operatorname{norm}\left(r(2 * t-1)-R 1 \_12(1)\right)\right)^{\wedge} 2\right)+((\operatorname{norm}(r(2 * t)-\right.$

R1 $\left.\left.13(2)))^{\wedge} 2\right)\right) ; Q(13, t 1 \overline{)}=1$;

ul $13(t 1)=(((\operatorname{norm}(r(2 * t-1)-R 113(1))) \wedge 2)+((\operatorname{norm}(r(2 * t)-$

$\left.\left.\left.\left.\mathrm{R}_{\text {__ }} 14(2)\right)\right)^{\wedge} 2\right)\right) ; \mathrm{Q}(14, \mathrm{t} 1 \overline{)}=1$;

ul_14 $(t 1)=\left(\left(\left(\operatorname{norm}\left(r(2 * t-1)-\mathrm{R} 1{ }_{-} 14(1)\right)\right)^{\wedge} 2\right)+((\operatorname{norm}(r(2 \star t)-\right.$

$\left.\left.\left.\left.\mathrm{R} 1 \_15(2)\right)\right)^{\wedge} 2\right)\right) ; \mathrm{Q}(15, \mathrm{t} 1 \overline{)}=1$;

u1_15 (t1) $=\left(\left(\left(\operatorname{norm}\left(r(2 * t-1)-\mathrm{R} 1_{-} 15(1)\right)\right)^{\wedge} 2\right)+((\operatorname{norm}(r(2 * t)-\right.$

R1_16(2)) )^2));Q(16,t1) $=1$;

end

if $Q(2, t)==1$

u2_1 $1(t)=\left(\left(\left(\operatorname{norm}\left(r(2 * t-1)-R 2{ }^{1}(1)\right)\right)^{\wedge} 2\right)+((\operatorname{norm}(r(2 * t)-\right.$ $\left.\left.\left.\left.\mathrm{R} 2 \_1(2)\right)\right)^{\wedge} 2\right)\right) ; \mathrm{Q}(1, \mathrm{t} 1) \overline{=}$;

u $2 \_2(t 1)=\left(\left(\left(\operatorname{norm}\left(r(2 * t-1)-R 2 z^{2}(1)\right)\right)^{\wedge} 2\right)+((\operatorname{norm}(r(2 * t)-\right.$ R2 2(2)) )^2)); Q (2, t 1$) \overline{=}$;

$u^{2} 3(t 1)=\left(\left(\left(\operatorname{norm}\left(r(2 * t-1)-\mathrm{R} 2{ }_{-} 3(1)\right)\right)^{\wedge} 2\right)+((\operatorname{norm}(r(2 * t)-\right.$

$\left.\left.\left.\left.\mathrm{R}^{2} 3(2)\right)\right)^{\wedge} 2\right)\right) ; \mathrm{Q}(3, \mathrm{t} 1)=1 ;$

$\left.\left.\left.\left.\mathrm{R} 2 \_4(2)\right)\right)^{\wedge} 2\right)\right) ; \quad Q(4, t 1) \overline{=}$;

$u^{2} 4(t 1)=\left(\left(\left(\operatorname{norm}\left(r(2 \star t-1)-\mathrm{R} 2{ }_{-} 4(1)\right)\right)^{\wedge} 2\right)+((\operatorname{norm}(r(2 \star t)-\right.$ 


\section{APPENDIX G (Continued)}

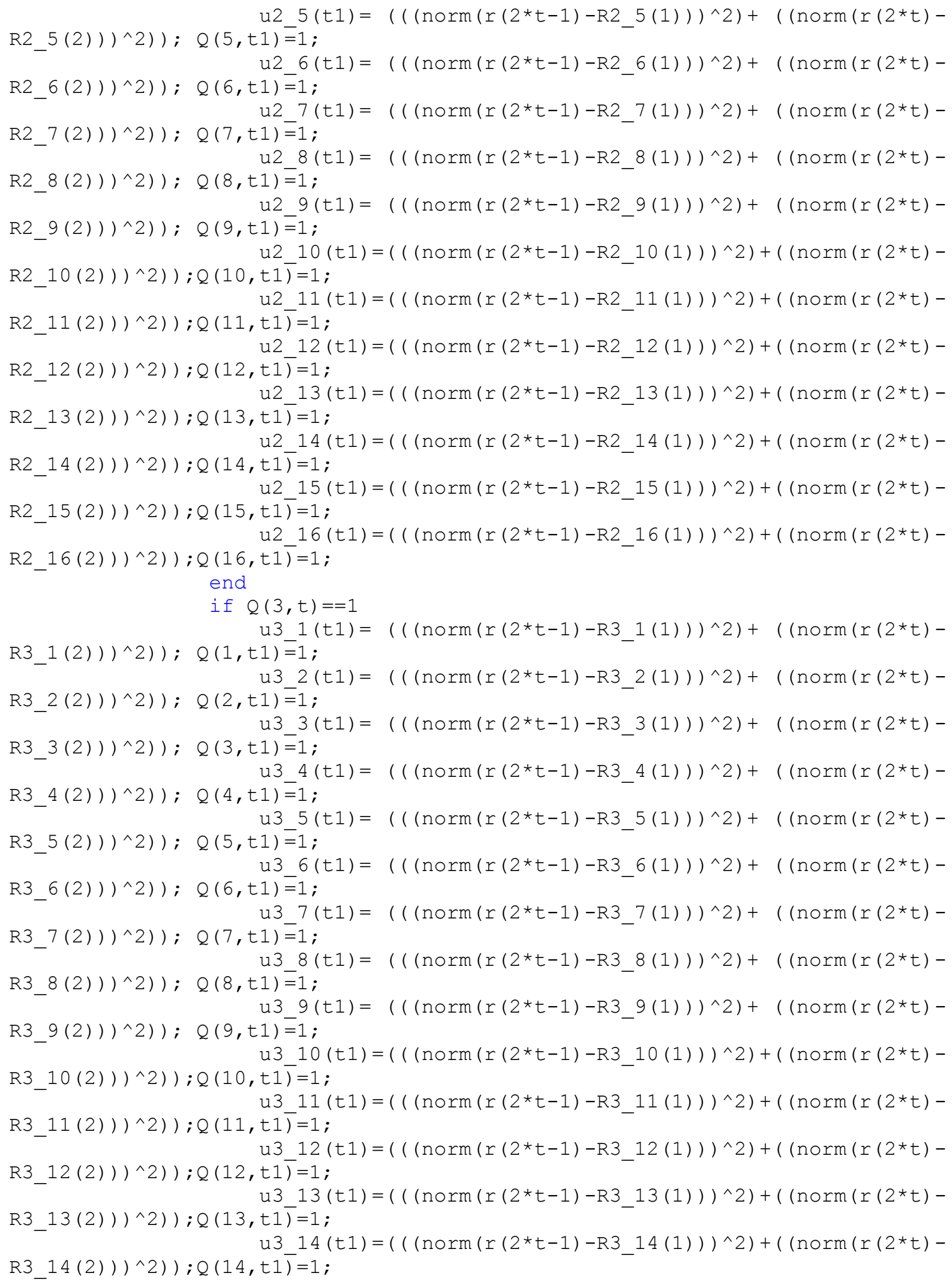




\section{APPENDIX G (Continued)}

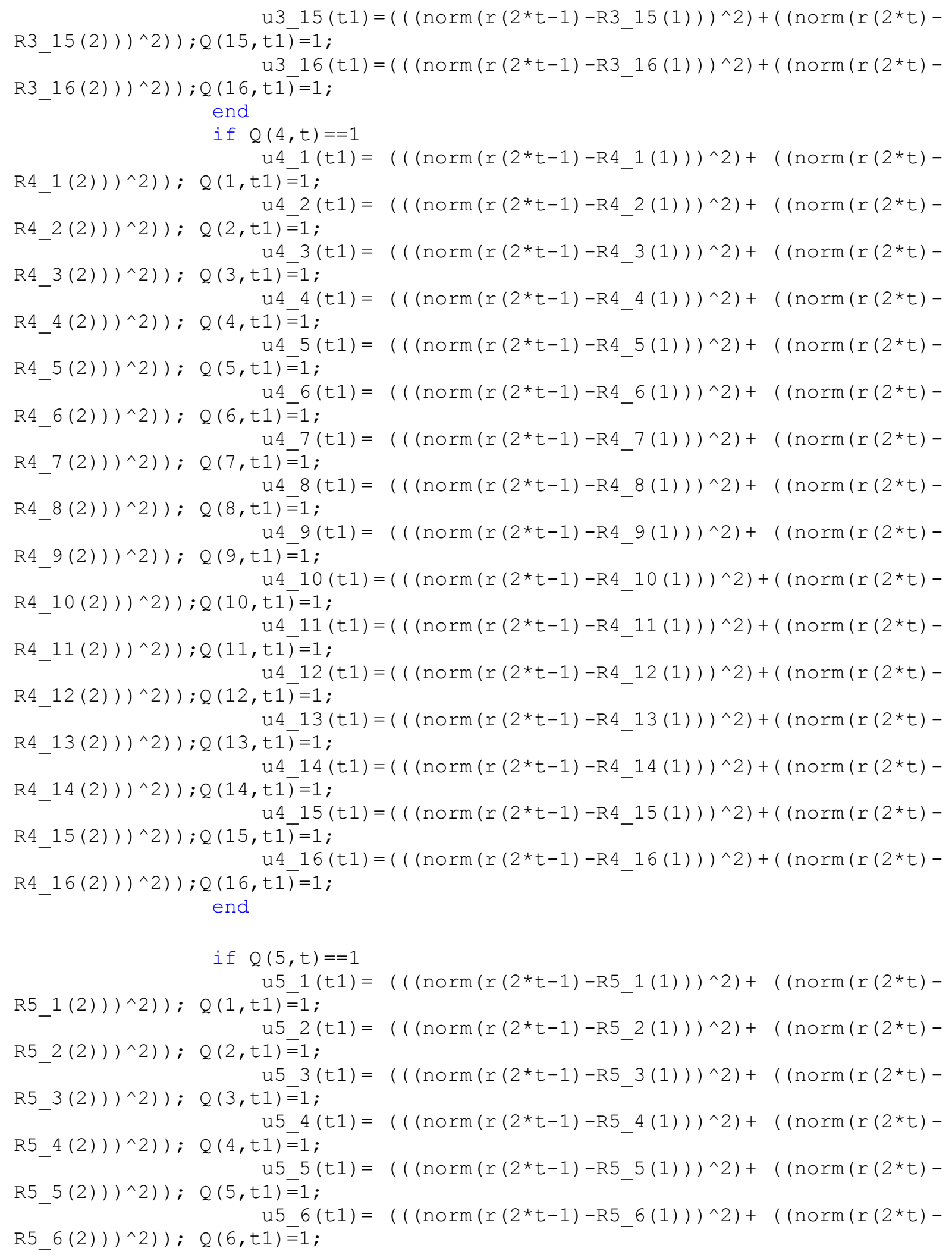




\section{APPENDIX G (Continued)}

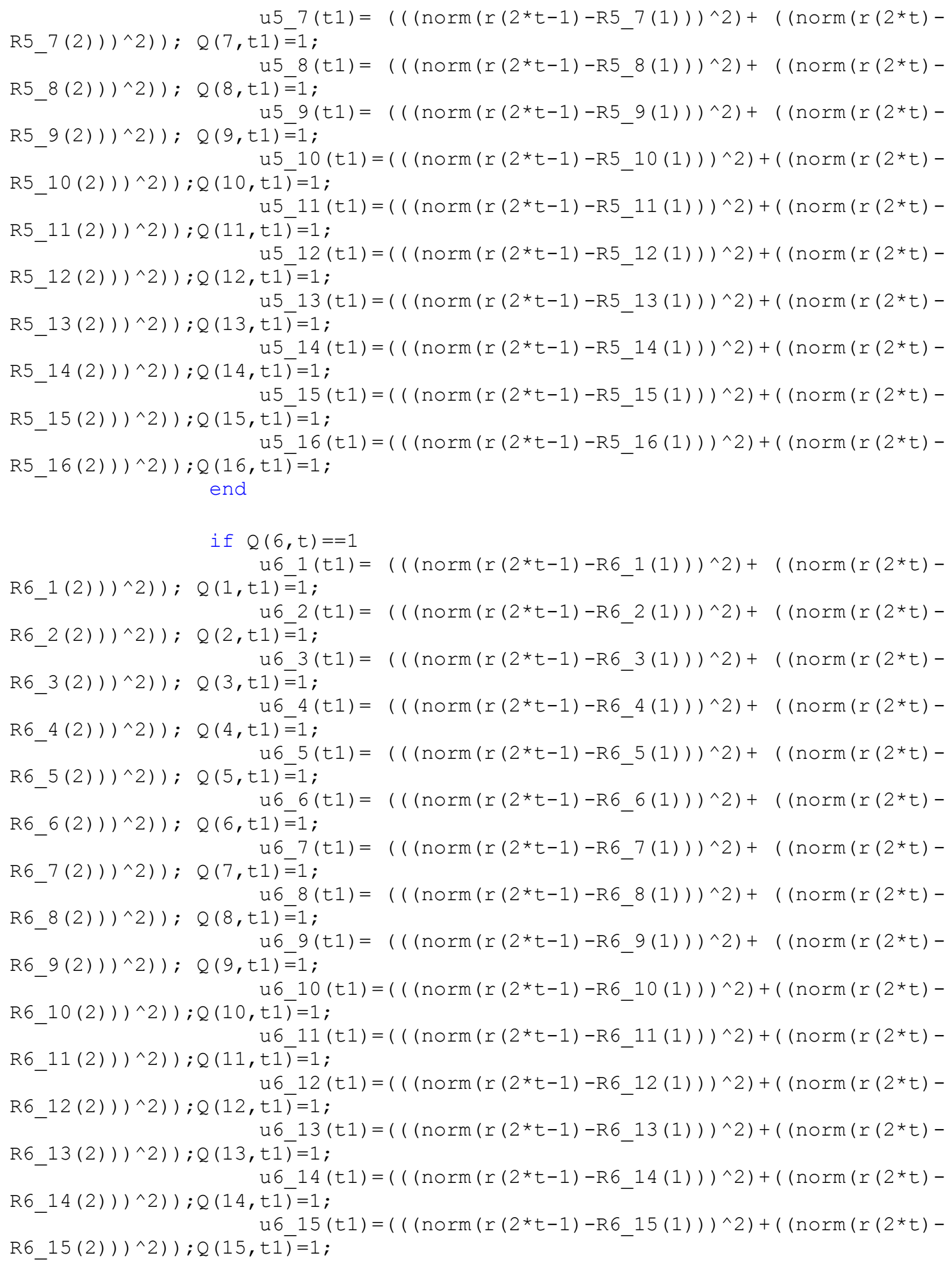




\section{APPENDIX G (Continued)}

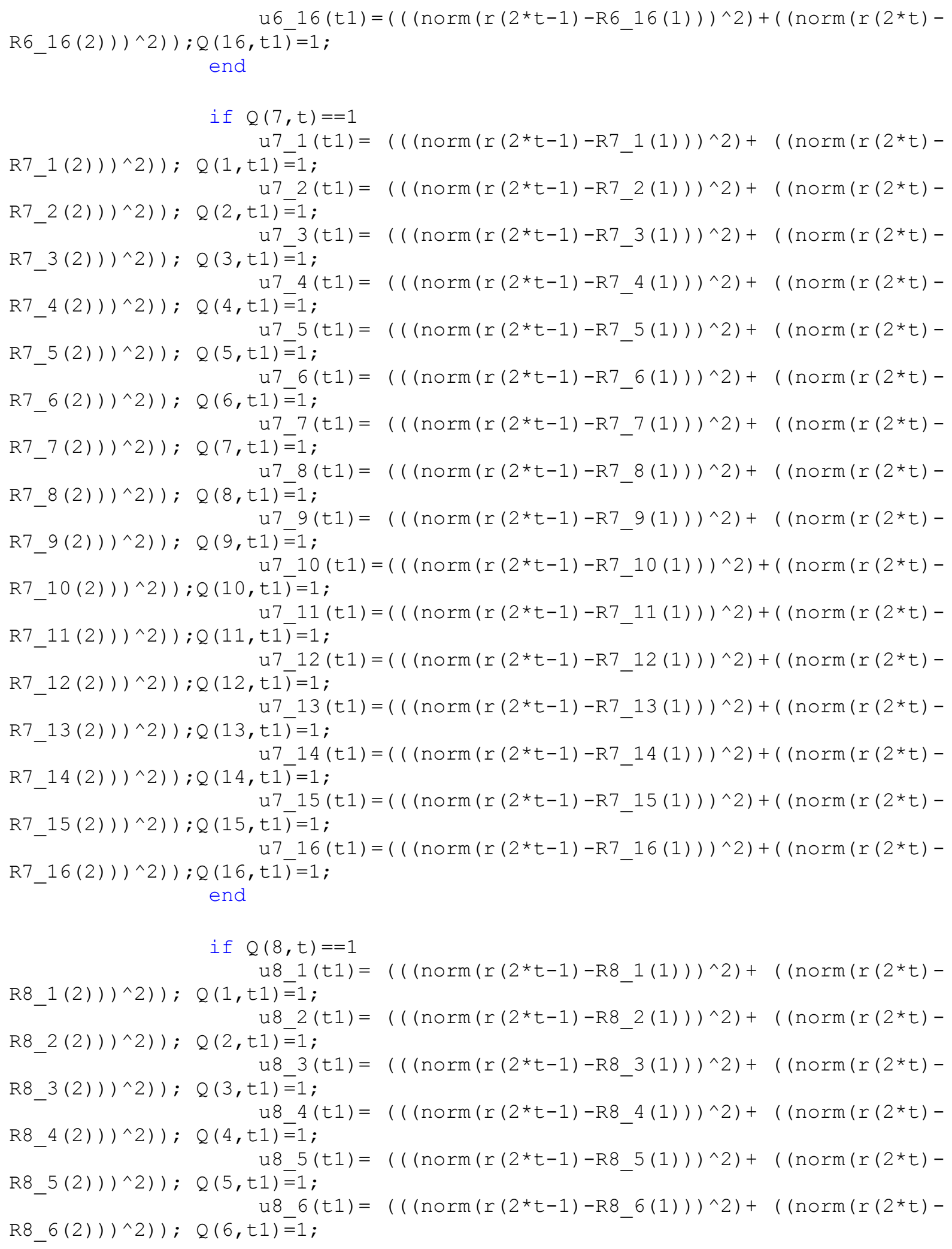




\section{APPENDIX G (Continued)}

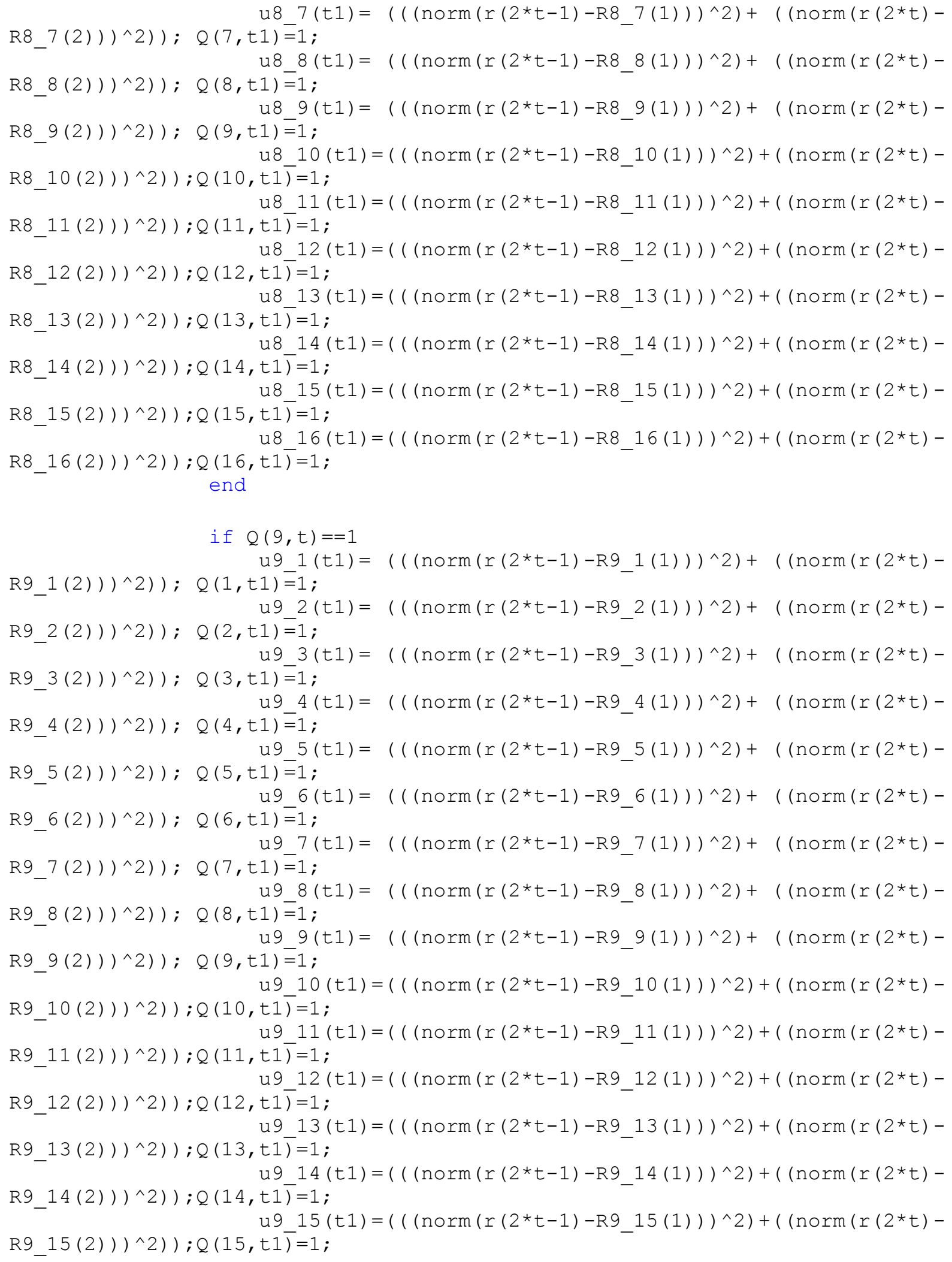




\section{APPENDIX G (Continued)}

R9_16(2)))^2));Q(16, t 1$)^{16}=1$;

u $916(t 1)=\left(\left((\operatorname{norm}(r(2 * t-1)-\operatorname{R} 916(1)))^{\wedge} 2\right)+((\operatorname{norm}(r(2 * t)-\right.$ end

if $Q(10, t)==1$

ulo $1(t 1)=\left(\left((\operatorname{norm}(r(2 * t-1)-\operatorname{R} 101(1)))^{\wedge} 2\right)+((\operatorname{norm}(r(2 * t)-\right.$

R10_1(2)) )^2)); Q (1, t1) $=1$; u10_2(t1) $=\left(\left(\left(\operatorname{norm}\left(r(2 * t-1)-R 10 \_2(1)\right)\right)^{\wedge} 2\right)+((\operatorname{norm}(r(2 * t)-\right.$

R10_2(2)) )^2)); $Q(2$, t 1$) \overline{=1}$; u10_3 $3(t)=\left(\left(\left(\operatorname{norm}\left(r(2 * t-1)-R 10 \_3(1)\right)\right)^{\wedge} 2\right)+((\operatorname{norm}(r(2 * t)-\right.$ R10_3(2)) )^2)); Q (3, t1) $=1$; u10 $4(t 1)=\left(\left((\operatorname{norm}(r(2 * t-1)-\operatorname{R10} 4(1)))^{\wedge} 2\right)+((\operatorname{norm}(r(2 * t)-\right.$ R10 $\left.\left.4(2)))^{\wedge} 2\right)\right) ; Q(4$, t 1$)=1$;

R10_5(2)) )^2)); Q (5, t1) $=1$; u10_5 (t 1$)=\left(\left(\left(\operatorname{norm}\left(r(2 * t-1)-\mathrm{R} 10 \_5(1)\right)\right)^{\wedge} 2\right)+((\operatorname{norm}(r(2 * t)-\right.$ R10 $\left.\left.6(2)))^{\wedge} 2\right)\right) ; Q(6, t 1)=1$; u10_6(t1) $=\left(\left(\left(\operatorname{norm}\left(r(2 * t-1)-\mathrm{R}_{10}{ }_{-} 6(1)\right)\right)^{\wedge} 2\right)+((\operatorname{norm}(r(2 * t)-\right.$ R10_7(2)) )^2)); $Q(7, t 1)=1$; R10_8(2)) )^2)); $Q(8, t 1)=1$; ulo_8 $($ t 1$)=\left(\left(\left(\operatorname{norm}\left(r(2 * t-1)-R 10 \_8(1)\right)\right) \wedge 2\right)+((\operatorname{norm}(r(2 * t)-\right.$ R10_9(2)) )^2)); $Q(9$, t 1$)=1$; u10_9 $9(t)=\left(\left(\left(\operatorname{norm}\left(r(2 * t-1)-R 10 \_9(1)\right)\right) \wedge 2\right)+((\operatorname{norm}(r(2 * t)-\right.$ R10_10(2)))^2)); $Q(10, t 1)=1$; ulo $10(t 1)=(((\operatorname{norm}(r(2 \star t-1)-\operatorname{R} 1010(1))) \wedge 2)+((\operatorname{norm}(r(2 \star t)-$ R10_11(2)) )^2)) ; Q (11, t1 $\overline{)}=1$; ulo_11 (t 1$)=\left(\left(\left(\operatorname{norm}\left(r(2 * t-1)-R 10 \_11(1)\right)\right)^{\wedge} 2\right)+((\operatorname{norm}(r(2 * t)-\right.$ R10_12(2)))^2));Q(12, t1) $=1$; R10_13(2)))^2)); $(13, t 1)=1$ u10 $13(t 1)=\left(\left((\operatorname{norm}(r(2 * t-1)-\operatorname{R} 1013(1)))^{\wedge} 2\right)+((\operatorname{norm}(r(2 * t)-\right.$ R10_14(2)) )^2)) ; Q (14, t1) $=1$; ulo_14 (t 1$)=\left(\left(\left(\operatorname{norm}\left(r(2 * t-1)-R_{10} 14(1)\right)\right) \wedge 2\right)+((\operatorname{norm}(r(2 \star t)-\right.$ R10_15(2)))^2));Q(15,t1) $=1$; R10_16(2)) )^2)) $: Q(16, t 1)^{16}=1$; end

if $Q(11, t)==1$

ul1_1(t1) $=\left(\left((\operatorname{norm}(r(2 * t-1)-\operatorname{R} 111(1)))^{\wedge} 2\right)+((\operatorname{norm}(r(2 * t)-\right.$ R11_1(2)) )^2)); $Q(1, t 1) \overline{=1}$; ul1_2(t1) $=\left(\left((\operatorname{norm}(r(2 * t-1)-\operatorname{R} 112(1)))^{\wedge} 2\right)+((\operatorname{norm}(r(2 * t)-\right.$ R11_2(2)))^2)); $Q(2, t 1) \overline{=}$; ul1_3(t1) $=\left(\left(\left(\operatorname{norm}\left(r(2 * t-1)-\operatorname{R} 11 \_3(1)\right)\right)^{\wedge} 2\right)+((\operatorname{norm}(r(2 \star t)-\right.$ R11_3(2)) )^2)); $Q(3, t 1)=1 ;$ ul1 $4(t 1)=\left(\left((\operatorname{norm}(r(2 * t-1)-\operatorname{R} 114(1)))^{\wedge} 2\right)+((\operatorname{norm}(r(2 * t)-\right.$ R11_4(2)) )^2)); $Q(4, \mathrm{t} 1)=1$; ul1_5 (t1) $=\left(\left((\operatorname{norm}(r(2 * t-1)-R 115(1)))^{\wedge} 2\right)+((\operatorname{norm}(r(2 * t)-\right.$ R11_5(2)) )^2)); $Q(5$, t 1$)=1 ;$ ul1_6(t1) $=\left(\left(\left(\operatorname{norm}\left(r(2 * t-1)-\operatorname{R} 11 \_6(1)\right)\right)^{\wedge} 2\right)+((\operatorname{norm}(r(2 * t)-\right.$ R11_6(2)))^2)); $Q(6, t 1)=1 ;$ ul1_7 (t1) $=\left(\left(\left(\operatorname{norm}\left(r(2 * t-1)-\operatorname{R} 11_{-} 7(1)\right)\right)^{\wedge} 2\right)+((\operatorname{norm}(r(2 * t)-\right.$ R11_7(2)) )^2)); $Q(7, t 1)=1 ;$ 


\section{APPENDIX G (Continued)}

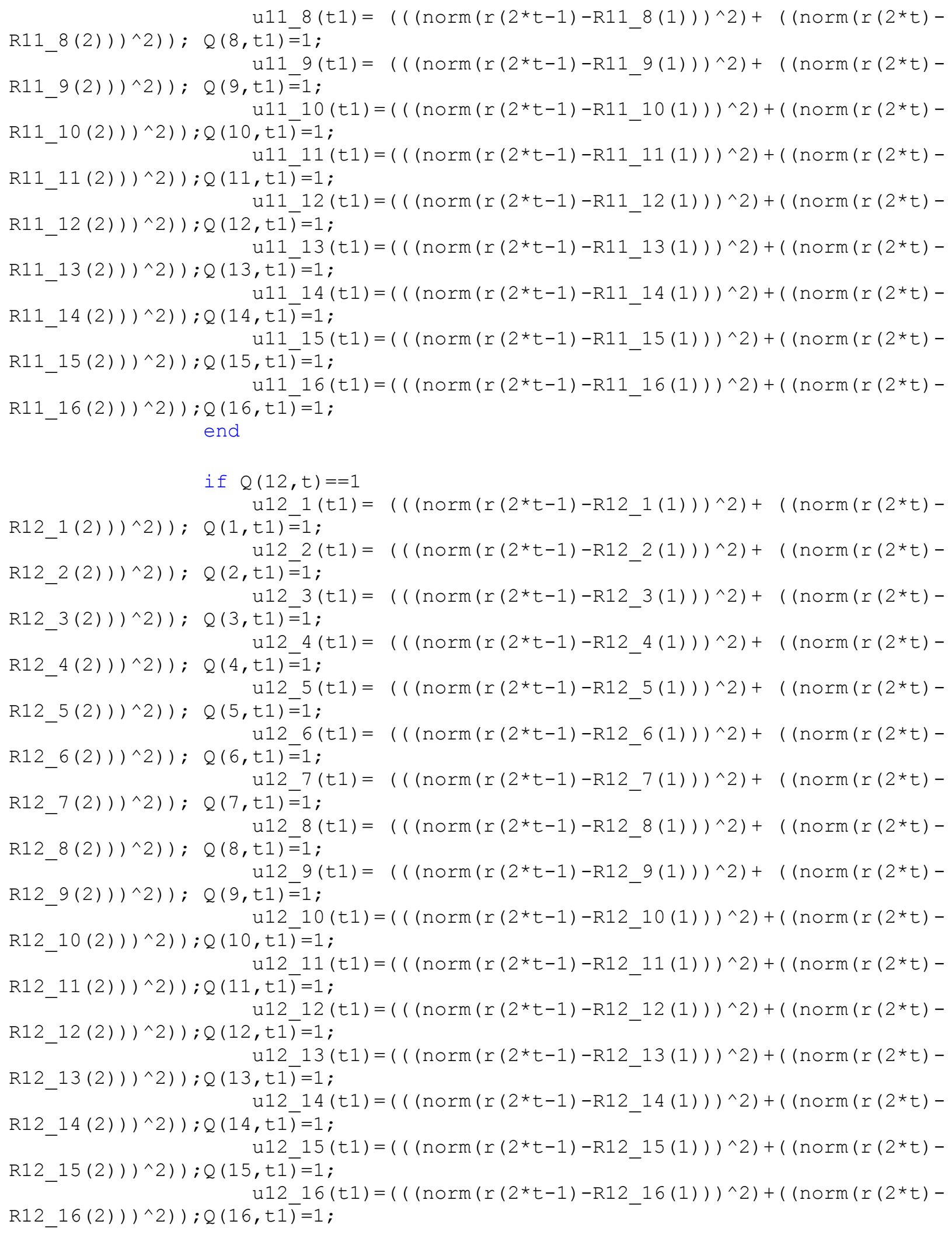




\section{APPENDIX G (Continued)}

end

if $Q(13, t)==1$

u13_1 (t1 $)=\left(\left(\left(\operatorname{norm}\left(r(2 * t-1)-\operatorname{R} 13 \_1(1)\right)\right)^{\wedge} 2\right)+((\operatorname{norm}(r(2 \star t)-\right.$

R13_1(2)) )^2)); $Q(1$, t 1$)=1$;

ul3 $2(t 1)=\left(\left((\operatorname{norm}(r(2 * t-1)-\operatorname{R13} 2(1)))^{\wedge} 2\right)+((\operatorname{norm}(r(2 * t)-\right.$

R13_2(2)) )^2)); $Q(2, t 1) \overline{=}$;

u13_3 $(t 1)=\left(\left(\left(\operatorname{norm}\left(r(2 * t-1)-\operatorname{R} 13 \_3(1)\right)\right)^{\wedge} 2\right)+((\operatorname{norm}(r(2 * t)-\right.$

R13_3(2)) )^2)); $Q(3, t 1)=1 ;$

u13_4 (t1) $=\left(\left(\left(\operatorname{norm}\left(r(2 * t-1)-\operatorname{R} 13 \_4(1)\right)\right)^{\wedge} 2\right)+((\operatorname{norm}(r(2 * t)-\right.$ R13_4(2)) )^2)); $Q(4, t 1)=\overline{1}$;

u13 $5(t 1)=\left(\left((\operatorname{norm}(r(2 * t-1)-\operatorname{R} 135(1)))^{\wedge} 2\right)+((\operatorname{norm}(r(2 * t)-\right.$ R13_5(2)) )^2)); $Q(5, t 1)=1 ;$ u13 $6(t 1)=\left(\left((\operatorname{norm}(r(2 * t-1)-\operatorname{R} 136(1)))^{\wedge} 2\right)+((\operatorname{norm}(r(2 * t)-\right.$ $\left.\left.\left.\left.\mathrm{R} 13 \_6(2)\right)\right)^{\wedge} 2\right)\right) ; \mathrm{Q}(6, t 1) \overline{=}$; u13_7 (t1) $=\left(\left(\left(\operatorname{norm}\left(r(2 * t-1)-\operatorname{R} 13 \_7(1)\right)\right)^{\wedge} 2\right)+((\operatorname{norm}(r(2 * t)-\right.$ R13 $\left.\left.7(2)))^{\wedge} 2\right)\right) ; Q(7, t 1)=1$; u13 $8(t 1)=\left(\left((\operatorname{norm}(r(2 * t-1)-\operatorname{R} 138(1)))^{\wedge} 2\right)+((\operatorname{norm}(r(2 * t)-\right.$ R13_8(2)) )^2)); $Q(8, t 1)=1 ;$

R13_9(2)) )^2)); Q $(9, t 1) \overline{=}$; u13_9 (t1) $=\left(\left(\left(\operatorname{norm}\left(r(2 * t-1)-\operatorname{R} 13{ }_{-} 9(1)\right)\right)^{\wedge} 2\right)+((\operatorname{norm}(r(2 * t)-\right.$ R13_10(2)))^2)); Q (10,t1) $=1$;

u13_10 (t1 $)=\left(\left(\left(\operatorname{norm}\left(r(2 * t-1)-R 13 \_10(1)\right)\right)^{\wedge} 2\right)+((\operatorname{norm}(r(2 * t)-\right.$ R13_11(2)) )^2));Q(11,t1) $=1$; u13_11 $(t 1)=\left(\left(\left(\operatorname{norm}\left(r(2 * t-1)-\operatorname{R} 13 \_11(1)\right)\right)^{\wedge} 2\right)+((\operatorname{norm}(r(2 * t)-\right.$ R13_12(2)) )^2)); Q (12, t1 $\overline{1}=1$; u13_12 $(t 1)=\left(\left(\left(\operatorname{norm}\left(r(2 * t-1)-\operatorname{R} 13 \_12(1)\right)\right)^{\wedge} 2\right)+((\operatorname{norm}(r(2 * t)-\right.$ R13_13(2)))^2)); Q (13, t $\overline{1} \overline{)}=1$;

R13_14(2)) )^2)); Q (14,t1) $=1$; u13 $14(t 1)=\left(\left((\operatorname{norm}(r(2 * t-1)-\operatorname{R} 1314(1)))^{\wedge} 2\right)+((\operatorname{norm}(r(2 * t)-\right.$ R13_15(2)) )^2));Q(15, t $\overline{1} \overline{)}=1$; u13_15 (t1) $=\left(\left(\left(\operatorname{norm}\left(r(2 * t-1)-\operatorname{R} 13 \_15(1)\right)\right)^{\wedge} 2\right)+((\operatorname{norm}(r(2 * t)-\right.$ R13_16(2)) )^2)); Q (16, t $1 \overline{)}=1$ end

if $Q(14, t)==1$ u14_1 (t1) $=\left(\left(\left(\operatorname{norm}\left(r(2 * t-1)-\operatorname{R} 14 \_1(1)\right)\right)^{\wedge} 2\right)+((\operatorname{norm}(r(2 * t)-\right.$ R14_1(2)) )^2)); $Q(1, t 1) \overline{=}$; ul4 $2(t 1)=\left(\left((\operatorname{norm}(r(2 * t-1)-\operatorname{R} 142(1)))^{\wedge} 2\right)+((\operatorname{norm}(r(2 * t)-\right.$ R14_2(2)) )^2)); $Q(2, t 1) \overline{=}$; ul4_3(t1) $=\left(\left(\left(\operatorname{norm}\left(r(2 * t-1)-\operatorname{R} 14 \_3(1)\right)\right)^{\wedge} 2\right)+((\operatorname{norm}(r(2 * t)-\right.$ R14_3(2)) )^2)); $Q(3, t 1)=\overline{1}$; ul4_4 (t1) $=\left(\left(\left(\operatorname{norm}\left(r(2 * t-1)-\operatorname{R} 14 \_4(1)\right)\right)^{\wedge} 2\right)+((\operatorname{norm}(r(2 * t)-\right.$ R14_4(2)) )^2)); $Q(4, t 1)=1 ;$ ul4 $5(t 1)=\left(\left((\operatorname{norm}(r(2 * t-1)-\operatorname{R} 145(1)))^{\wedge} 2\right)+((\operatorname{norm}(r(2 * t)-\right.$ R14_5(2)))^2)); $Q(5, t 1)=1 ;$ ul4_6 (t1) $=\left(\left(\left(\operatorname{norm}\left(r(2 * t-1)-R 14 \_6(1)\right)\right) \wedge 2\right)+((\operatorname{norm}(r(2 * t)-\right.$ $\left.\left.\left.\left.\mathrm{R} 14 \_6(2)\right)\right)^{\wedge} 2\right)\right) ; \mathrm{Q}(6, \mathrm{t} 1) \overline{=}$; R14 $\left.\left.7(2)))^{\wedge} 2\right)\right) ; Q(7, t 1) \overline{=1}$; u14_7 (t1) $=\left(\left(\left(\operatorname{norm}\left(r(2 * t-1)-\operatorname{R} 14{ }_{-} 7(1)\right)\right)^{\wedge} 2\right)+((\operatorname{norm}(r(2 * t)-\right.$ R14_8(2)) )^2)); $Q(8, t 1) \overline{=} 1$; ul4_8 (t1) $=\left(\left(\left(\operatorname{norm}\left(r(2 \star t-1)-R 14 \_8(1)\right)\right)^{\wedge} 2\right)+((\operatorname{norm}(r(2 \star t)-\right.$ 


\section{APPENDIX G (Continued)}

u14_9 (t1) $=\left(\left(\left(\operatorname{norm}\left(r(2 * t-1)-R 14 \_9(1)\right)\right)^{\wedge} 2\right)+((\operatorname{norm}(r(2 * t)-\right.$ R14_9(2)) )^2)); $\mathrm{Q}(9, \mathrm{t} 1) \overline{=}$;

u14_10 (t1) $=\left(\left(\left(\operatorname{norm}\left(r(2 * t-1)-\operatorname{R} 14 \_10(1)\right)\right)^{\wedge} 2\right)+((\operatorname{norm}(r(2 * t)-\right.$ R14_10(2)))^2)); Q (10,t1) $=1$; u14_11 $(t 1)=\left(\left(\left(\operatorname{norm}\left(r(2 * t-1)-\operatorname{R} 14 \_11(1)\right)\right)^{\wedge} 2\right)+((\operatorname{norm}(r(2 \star t)-\right.$ R14_11(2)) )^2));Q(11, t1) $=1$; u14_12(t1) $=\left(\left(\left(\operatorname{norm}\left(r(2 * t-1)-\operatorname{R} 14 \_12(1)\right)\right)^{\wedge} 2\right)+((\operatorname{norm}(r(2 * t)-\right.$ R14_12(2)))^2)); $Q(12, t 1 \overline{)}=1$; u14 $13(t 1)=(((\operatorname{norm}(r(2 * t-1)-\operatorname{R} 1413(1))) \wedge 2)+((\operatorname{norm}(r(2 * t)-$ R14_13(2)))^2)); Q (13,t1) $=1$; R14_14(2)) )^2));Q(14,t1) $=1$; u14_14 $(t 1)=\left(\left(\left(\operatorname{norm}\left(r(2 * t-1)-\operatorname{R} 14 \_14(1)\right)\right)^{\wedge} 2\right)+((\operatorname{norm}(r(2 * t)-\right.$ R14_15(2)) )^2));Q(15, t $1 \overline{)}=1$; u14_15 $(t 1)=\left(\left((\operatorname{norm}(r(2 * t-1)-\operatorname{R} 14 \text { _ } 15(1)))^{\wedge} 2\right)+((\operatorname{norm}(r(2 * t)-\right.$ R14_16(2)) )^2));Q(16, t $1 \overline{)}=1$ end

if $Q(15, t)==1$ u15_1 $(t 1)=\left(\left(\left(\operatorname{norm}\left(r(2 * t-1)-\operatorname{R} 15 \_1(1)\right)\right)^{\wedge} 2\right)+((\operatorname{norm}(r(2 \star t)-\right.$ $\left.\left.\operatorname{R15} 1(2)))^{\wedge} 2\right)\right) ; \mathrm{Q}(1, \mathrm{t} 1) \overline{1}=$ u152 $2(t 1)=\left(\left((\operatorname{norm}(r(2 * t-1)-\operatorname{R} 152(1)))^{\wedge} 2\right)+((\operatorname{norm}(r(2 * t)-\right.$ R15_2(2)) )^2)); $Q(2, t 1) \overline{=}$;

R15_3(2)) )^2)); $Q(3, t 1) \overline{=}$; u15_3 $(t 1)=\left(\left(\left(\operatorname{norm}\left(r(2 \star t-1)-\operatorname{R} 15 \_3(1)\right)\right)^{\wedge} 2\right)+((\operatorname{norm}(r(2 \star t)-\right.$ R15 4 (2)) )^2)); Q (4,t1) $=1$; u15_4 $(t 1)=\left(\left(\left(\operatorname{norm}\left(r(2 * t-1)-\operatorname{R} 15 \_4(1)\right)\right)^{\wedge} 2\right)+((\operatorname{norm}(r(2 * t)-\right.$ u15 $5(t 1)=(((\operatorname{norm}(r(2 * t-1)-\operatorname{Ri} 55(1))) \wedge 2)+((\operatorname{norm}(r(2 * t)-$ R15_5(2)) )^2)); $Q(5$, t 1$)=1 ;$ u15_6 (t1) $=\left(\left(\left(\operatorname{norm}\left(r(2 * t-1)-\operatorname{R} 15 \_6(1)\right)\right)^{\wedge} 2\right)+((\operatorname{norm}(r(2 \star t)-\right.$ R15_6(2)) )^2)); Q $(6, t 1) \overline{=}$; $\left.\left.\operatorname{R} 157(2)))^{\wedge} 2\right)\right) ; Q(7, t 1)=1 ;$ u15 $8(t 1)=\left(\left((\operatorname{norm}(r(2 * t-1)-\operatorname{R} 158(1)))^{\wedge} 2\right)+((\operatorname{norm}(r(2 * t)-\right.$ R15_8(2)))^2)); $Q(8, t 1)=1 ;$ u15_9 $(t 1)=\left(\left(\left(\operatorname{norm}\left(r(2 * t-1)-\operatorname{R} 15 \_9(1)\right)\right)^{\wedge} 2\right)+((\operatorname{norm}(r(2 * t)-\right.$ R15_9(2)) )^2)); $Q(9$, t 1$) \overline{=} 1$; u15 $10(t 1)=\left(\left((\operatorname{norm}(r(2 * t-1)-\operatorname{R} 1510(1)))^{\wedge} 2\right)+((\operatorname{norm}(r(2 * t)-\right.$ R15_10(2)))^2)); Q (10,t1) $=1$; u15_11 $(t 1)=\left(\left(\left(\operatorname{norm}\left(r(2 * t-1)-\operatorname{R} 15 \_11(1)\right)\right)^{\wedge} 2\right)+((\operatorname{norm}(r(2 * t)-\right.$ R15_11(2)))^2)); $Q(11, t 1 \overline{)}=1$; u15_12(t1) $=\left(\left(\left(\operatorname{norm}\left(r(2 * t-1)-\operatorname{R} 15 \_12(1)\right)\right)^{\wedge} 2\right)+((\operatorname{norm}(r(2 * t)-\right.$ R15 $\left.\left.12(2)))^{\wedge} 2\right)\right) ; Q(12, t 1 \overline{)}=1$; u15_13 1 t1 $)=\left(\left(\left(\operatorname{norm}\left(r(2 * t-1)-\operatorname{R} 15 \_13(1)\right)\right)^{\wedge} 2\right)+((\operatorname{norm}(r(2 * t)-\right.$ R15_13(2)))^2)); Q (13, t $1 \overline{)}=1$; u15_14 (t1 $)=\left(\left(\left(\operatorname{norm}\left(r(2 * t-1)-\operatorname{R} 15 \_14(1)\right)\right)^{\wedge} 2\right)+((\operatorname{norm}(r(2 * t)-\right.$ R15_14(2)))^2)); $Q(14, t 1 \overline{)}=1$; u15_15 $(t 1)=\left(\left(\left(\operatorname{norm}\left(r(2 * t-1)-\operatorname{R} 15 \_15(1)\right)\right)^{\wedge} 2\right)+((\operatorname{norm}(r(2 * t)-\right.$ R15_15(2)) )^2));Q(15, t1 $\overline{)}=1$; u15_16 (t1 $)=\left(\left(\left(\operatorname{norm}\left(r(2 * t-1)-\operatorname{R} 15 \_16(1)\right)\right)^{\wedge} 2\right)+((\operatorname{norm}(r(2 * t)-\right.$ R15_16(2)))^2));Q(16,t1) $=1$; end 


\section{APPENDIX G (Continued)}

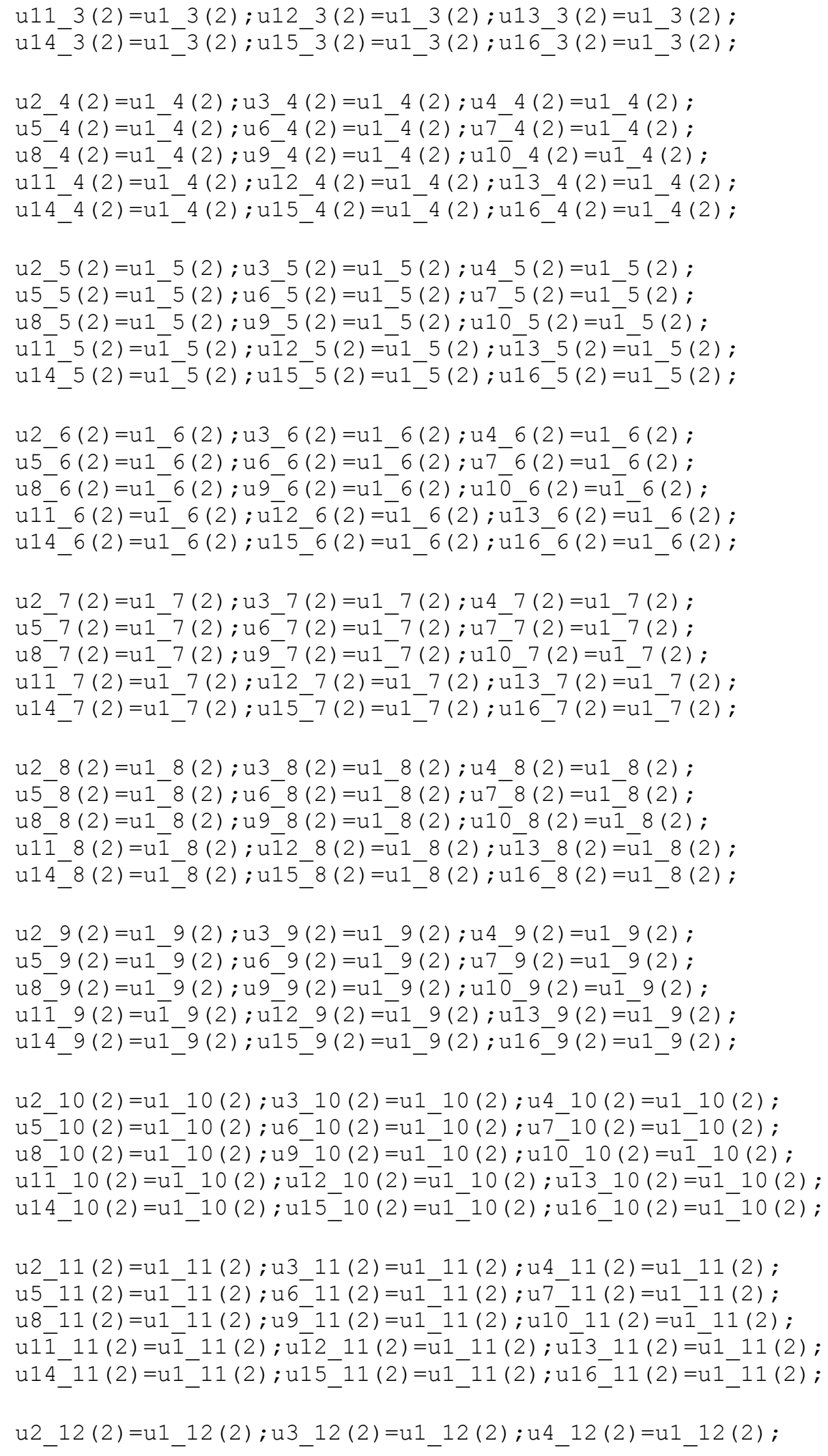




\section{APPENDIX G (Continued)}

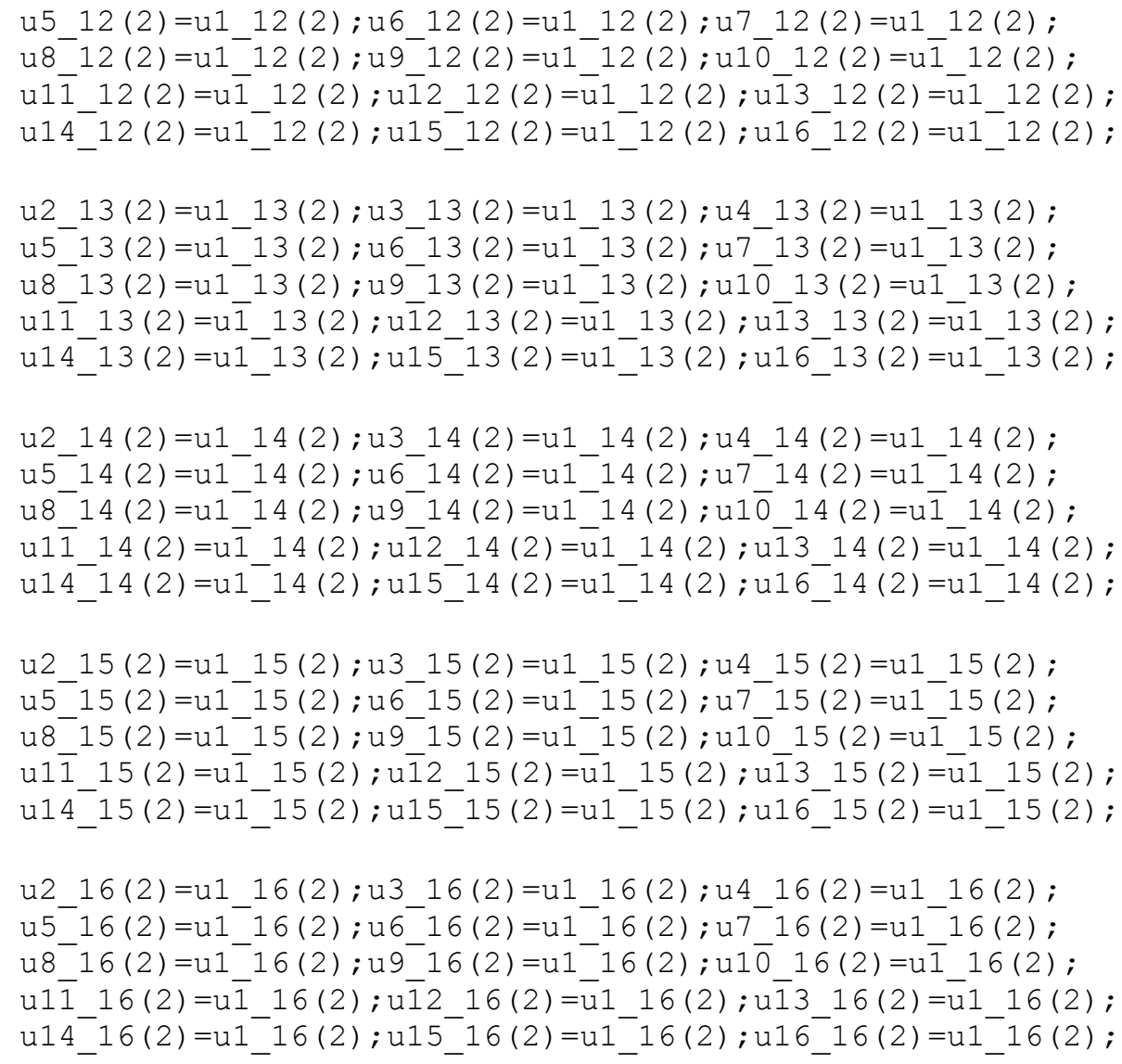

end

$j h 1=[\operatorname{mu} 1(t)+u 1 \quad 1(t 1) \quad \operatorname{mu} 2(t)+u 2 \quad 1(t 1) \quad \operatorname{mu} 3(t)+u 31(t 1)$

mu4 (t) +u4_1(t1) mu5 (t) +u5_1(t1) mu6 (t) +u6_1(t1) mu7 (t) +u7_1(t1)

mu8 $(t)+u 8$ 1 (t1) mug(t) +u9_1 (t1) mu10 (t) +u10_1(t1) mu11 (t) $+u 11$ 1 (t1)

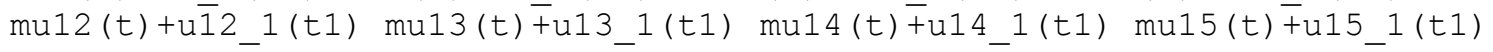

$\left.\operatorname{mu16}(\mathrm{t})+\mathrm{u} 16_{-}{ }_{1}(\mathrm{t} 1)\right]$;

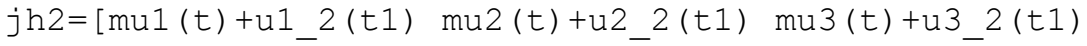

mu4 (t) +u4_2(t1) mu5 (t) +u5_2(t1) mu6 (t) +u6_2(t1) mu7 (t) +u7_2(t1)

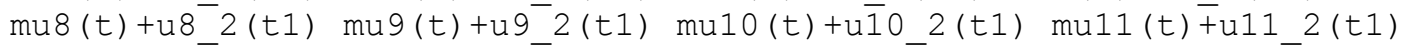

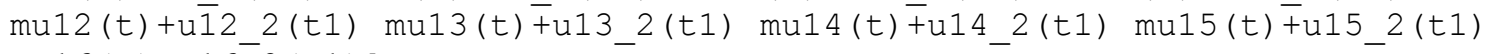

$\left.\operatorname{mu16}(t)+u 16 \_2(t 1)\right]$

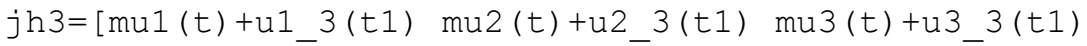

mu4 $(t)+u 4 \_3(t 1) \operatorname{mu} 5(t)+u 5 \_3(t 1) \operatorname{mu} 6(t)+u 6 \_3(t 1)$ mu7 (t) +u7_3(t1)

mu8 $(t)+u 8$ 3 (t1) mu9(t) +u9_3(t1) mu10 (t) +u10_3(t1) mu11 (t) +u11_3(t1)

$\operatorname{mu12}(t)+u \overline{1} 2 \_3(t 1) \operatorname{mul3}(t) \overline{+u 13} 3(t 1) \operatorname{mul4}(t) \overline{+u 14} 3(t 1)$ mu15(t) $+u 15$ 3 (t1)

$\left.\operatorname{mul6}(t)+u 16 \_3(t 1)\right]$

$j h 4=\left[\operatorname{mu} 1(t)+u 1_{-} 4(t 1) \quad m u 2(t)+u 2_{2} 4(t 1) \quad\right.$ mu3 $(t)+u 3{ }^{4} 4(t 1)$

$\operatorname{mu} 4(t)+u 44_{(t 1)} \operatorname{mu} 5(t)+u 5 \_4(t 1)^{-} \operatorname{mu} 6(t)+u 6 \_4(t 1) \operatorname{mu} 7(t)+u 7+4(t 1)$

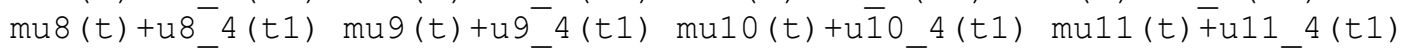




\section{APPENDIX G (Continued)}

$\operatorname{mu12}(t)+u 1244(t 1) \operatorname{mu13}(t)+u 13 \_4(t 1) \operatorname{mu14}(t)+u 14 \_4(t 1) \operatorname{mu15}(t)+u 15 \_4(t 1)$ $\left.\operatorname{mu16}(t)+u 16 \_4(t 1)\right] ;$

$j h 5=[\operatorname{mu} 1(t)+u 155(t 1) \quad$ mu2 $(t)+u 2$ 5 (t1) mu3 (t) +u3 $5(t 1)$

$\operatorname{mu} 4(t)+u 45(t 1) \operatorname{mu} 5(t)+u 55(t 1) \operatorname{mu} 6(t)+u 6 \quad 5(t 1) \operatorname{mu} 7(t)+u 75(t 1)$

mu8 $(t)+u 8-5(t 1)$ mug (t) +u9_5(t1) mulo (t) +u10_5 (t1) mul1 (t) +u11_5 (t1)

mu12 (t) +u12_5(t1) mu13(t) +u13_5(t1) mu14(t) +u14_5(t1) mu15(t) +u15_5(t1) mu16(t)+u16_5(t1)];

$j h 6=[\operatorname{mu} 1(t)+u 1 \quad 6(t 1) \quad \operatorname{mu} 2(t)+u 2 \quad 6(t 1) \quad \operatorname{mu} 3(t)+u 3 \quad 6(t 1)$

$\operatorname{mu} 4(t)+u 4$ 6(t1) mu5(t)+u5_6(t1) mu6(t)+u6 6(t1) mu7 (t) +u7 6(t1)

mu8 $(t)+u 8^{-} 6(t 1) \quad \operatorname{mu} 9(t)+u 9^{-} 6(t 1) \operatorname{mul0}(t)+u \overline{1} 06(t 1) \operatorname{mul1}(t) \overline{-}+u 116(t 1)$

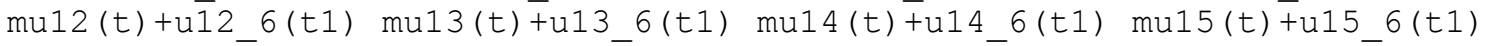
$\operatorname{mu16}(t)+u 16-6(t 1)] ;$

$j h 7=\left[\operatorname{mul}(t)+u 1_{-} 7(t 1) \quad \operatorname{mu} 2(t)+u 2_{-} 7(t 1) \quad \operatorname{mu} 3(t)+u 3{ }_{7} 7(t 1)\right.$

$\operatorname{mu} 4(t)+u 4 \quad 7(t 1) \operatorname{mu} 5(t)+u 57(t 1) \operatorname{mu} 6(t)+u 67(t 1) \operatorname{mu} 7(t)+u 7 \quad 7(t 1)$

$\operatorname{mu} 8(t)+u 8^{-} 7(t 1) \quad \operatorname{mug}(t)+u 9^{-} 7(t 1) \operatorname{mul0}(t)+u \overline{1} 07(t 1) \operatorname{mul1}(t) \overline{-} u 11 \quad 7(t 1)$

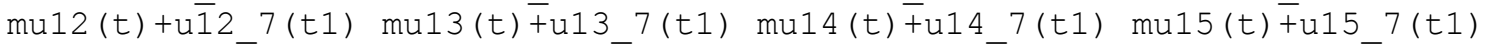

$\left.\operatorname{mu16}(t)+u 16^{-} 7(t 1)\right] ;$

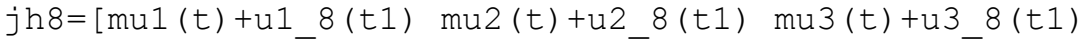

$\operatorname{mu} 4(t)+u 48$ (t1) mu5 (t) +u5_8(t1) mu6(t) +u6 8(t1) mu7 (t) +u7 8 (t1)

$\operatorname{mu} 8(t)+u 888(t 1) \operatorname{mu} 9(t)+u 9-8(t 1) \operatorname{mu} 10(t)+u \overline{1} 08$ (t 1$) \operatorname{mul1}(t)+u 118$ (t1)

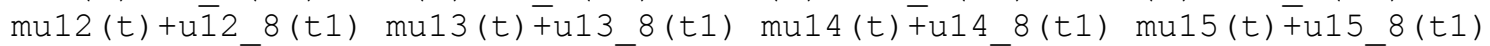

mu16(t)+u16_8(t1)] ;

jh $9=[\operatorname{mu} 1(t)+u 1 \quad 9(t 1)$ mu2 (t) +u2 $9(t 1)$ mu3 (t) +u3 $9(t 1)$

mu4 (t) +u4_9(t1) mu5 (t) +u5_9(t1) mu6 (t) +u6_9(t1) mu7 (t) +u7_9(t1)

mu8 (t) +u8_9(t1) mug(t) +u9_9(t1) mu10 (t) +u10_9(t1) mu11 (t) +u11_9(t1)

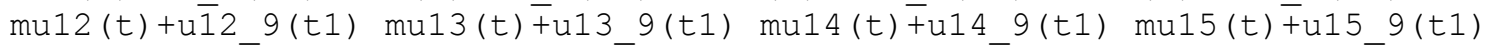

mu16(t)+u16_9(t1)];

$j h 10=[\operatorname{mu} 1(t)+u 1 \quad 10(t 1) \quad \operatorname{mu} 2(t)+u 2 \quad 10(t 1) \quad \operatorname{mu} 3(t)+u 3 \quad 10(t 1)$

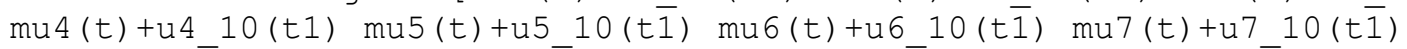

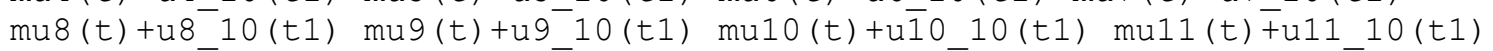

mu12 (t) +u1̄2_10(t1) mu13(t) +u13_10(t1) mu14(t) +u14_10(t1) mu15(t) +u15_10(t1)

$\operatorname{mu16}(t)+u 16-10(t 1)] ;$

$j h 11=[\operatorname{mu} 1(t)+u 1 \quad 11(t 1) \quad \operatorname{mu} 2(t)+u 2 \quad 11(t 1) \quad \operatorname{mu} 3(t)+u 3 \quad 11(t 1)$

$\operatorname{mu} 4(t)+u 4 \_11(t 1) \quad \operatorname{mu} 5(t)+u 5 \_11(t \overline{1}) \quad \operatorname{mu} 6(t)+u 6 \_11(t \overline{1})$ mu7 $(t)+u 7 \_11(t \overline{1})$

mu8 $(t)+u 8-11(t 1)$ mu9 (t) +u9 $11(t 1)$ mu10 (t) +u10_11(t1) mu11 (t) +u11_11(t1)

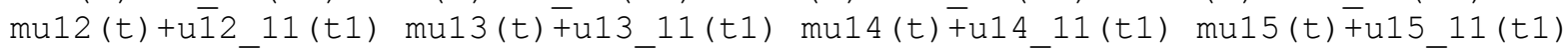

$\left.\operatorname{mu16}(t)+u 16 \_11(t 1)\right] ;$

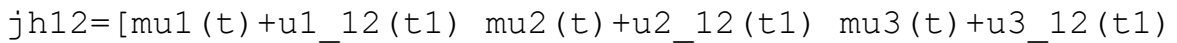

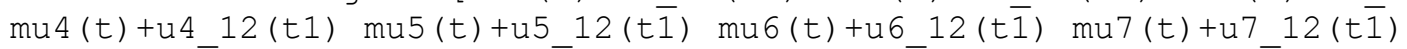

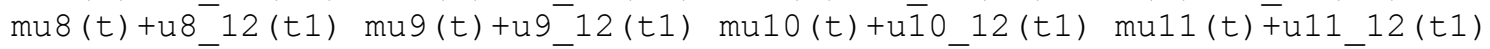

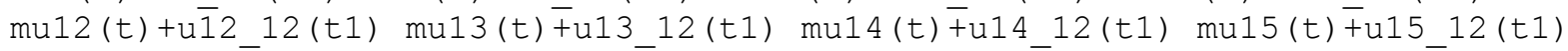

$\left.\operatorname{mu16}(\mathrm{t})+\mathrm{u} 16_{-} 12(\mathrm{t} 1)\right]$;

$j h 13=\left[\operatorname{mul}(t)+u 1 \_13(t 1) \quad\right.$ mu2 $(t)+u 2 \_13(t 1)$ mu3 (t) +u3 13(t1)

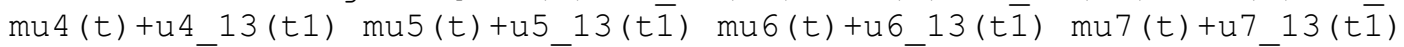

mu8 $(t)+u 8$ 13 (t1) mu9 (t) +u9_13(t1) mu10 (t) +u10_13(t1) mu11(t) +u11_13(t1)

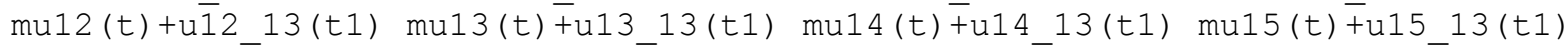

$\left.\operatorname{mu16}(t)+u 16 \_13(t 1)\right]$;

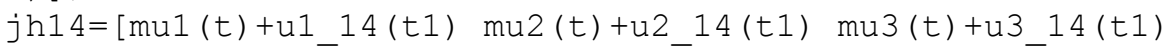

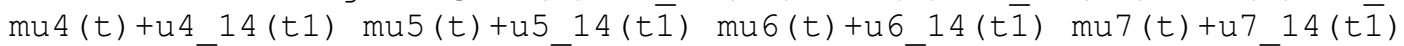

mu8 $(t)+u 814(t 1) \operatorname{mug}(t)+u 9^{-14}(t 1) \operatorname{mul0}(t)+u \overline{1} 014(t 1)$ mul1 (t) +u11_14 (t1)

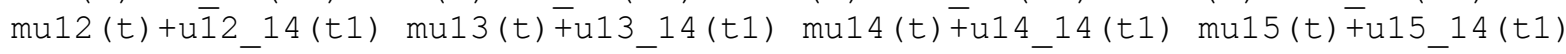

$\left.\operatorname{mu} 16(t)+u 16 \_14(t 1)\right] ;$

jh15 $=\left[\operatorname{mul}(t)+u 1 \_15(t 1)\right.$ mu2 (t) +u2_15(t1) mu3 (t) +u3_15(t1)

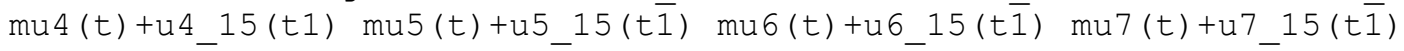




\section{APPENDIX G (Continued)}

mu8 (t) +u8 $15(t 1) \quad \operatorname{mu} 9(t)+u 9 \quad 15(t 1) \quad \operatorname{mu} 10(t)+u 10 \quad 15(t 1) \operatorname{mul1}(t)+u 11 \quad 15$ (t1) $\operatorname{mu12}(t)+u 12 \_15(t 1) \operatorname{mul3}(t)+u 13 \_15(t 1) \operatorname{mul4}(t)+u 14 \_15(t 1) \operatorname{mu} 15(t)+u 15$ 15 (t1) $\left.\operatorname{mu16}(\mathrm{t})+\mathrm{u} 16_{-} 15(\mathrm{t} 1)\right]$; $j h 16=[\operatorname{mu} 1(t)+u 116(t 1) \quad \operatorname{mu} 2(t)+u 2 \quad 16(t 1) \quad \operatorname{mu} 3(t)+u 3 \quad 16(t 1)$

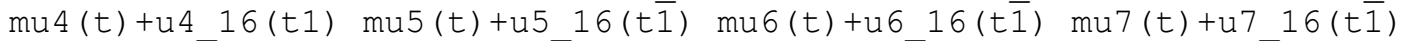
mu8 $(t)+u 8-16(t 1) \quad \operatorname{mug}(t)+u 9-16(t 1)$ mu10 (t) +u10_16(t1) mu11 (t) +u11_16(t1) mu12 (t) +u12_16(t1) mu13(t) +u13_16(t1) mu14(t) +u14_16(t1) mu15(t) +u15_16(t1) $\left.\operatorname{mu16}(t)+u 16 \_16(t 1)\right] ;$

$\operatorname{mu} 1(t 1)=\min (j h 1) ; \operatorname{mu} 2(t 1)=\min (j h 2) ; \operatorname{mu} 3(t 1)=\min (j h 3) ; \operatorname{mu} 4(t 1)=\min (j h 4) ; \operatorname{mu} 5(t 1)=m$ in $(j h 5) ; \operatorname{mu} 6(t 1)=\min (j h 6) ; \operatorname{mu} 7(t 1)=\min (j h 7) ; \operatorname{mu} 8(t 1)=\min (j h 8)$;

mu9 $(t 1)=\min (j h 9) ; \operatorname{mul0}(t 1)=\min (j h 10) ; \operatorname{mul1}(t 1)=\min (j h 11) ; \operatorname{mu} 12(t 1)=\min (j h 12) ; \operatorname{mu} 1$ $3(t 1)=\min (j h 13) ; \operatorname{mul} 4(t 1)=\min (j h 14) ; \operatorname{mul5}(t 1)=\min (j h 15) ; \operatorname{mu} 16(t 1)=\min (j h 16) ;$

$\circ g \circ 1$

if $\operatorname{mul}(t 1)==\operatorname{mul}(t)+u 1 \quad 1(t 1)$

$g \circ 1(t)=1$;

elseif $\operatorname{mu} 1(t)==\operatorname{mu} 2(t)+u 2 \_1(t 1)$ $g \circ 1(t)=2$;

elseif mu1 (t 1$)==\operatorname{mu} 3(t)+u 3 \_1(t 1)$ $g \circ 1(t)=3$;

elseif mul (t 1$)==m u 4(t)+u 4 \quad 1(t 1)$ $g \circ 1(t)=4$;

elseif mul (t 1$)==m u 5(t)+u 5$ _ (t 1$)$ $g \circ 1(t)=5$;

elseif mul (t 1$)==m u 6(t)+u \sigma_{-} 1(t 1)$ $g \circ 1(t)=6$;

elseif mul (t 1$)==\operatorname{mu} 7(t)+u 7 \_1(t 1)$ $g \circ 1(t)=7$;

elseif mul (t 1$)==m u 8(t)+u 8 \_1(t 1)$ $g \circ 1(t)=8$;

elseif mul (t 1$)==\operatorname{mu} 9(t)+u 9 \_1(t 1)$ $g \circ 1(t)=9$;

elseif mul $(t 1)==\operatorname{mu} 10(t)+u 10 \_1(t 1)$ $g \circ 1(t)=10$;

elseif mul (t 1$)==\operatorname{mul1}(t)+u 11 \_1(t 1)$ $g \circ 1(t)=11 ;$

elseif $\operatorname{mul}(t 1)==\operatorname{mu} 12(t)+u 12 \quad 1(t 1)$ $g \circ 1(t)=12$;

elseif mul (t 1$)==\operatorname{mu13}(t)+u 13 \_1(t 1)$ $g \circ 1(t)=13$;

elseif mul $(t 1)==\operatorname{mu14}(t)+u 14 \_1(t 1)$ go1 $(t)=14$;

elseif mul (t 1$)==\operatorname{mu15}(t)+u 15 \_1(t 1)$ $g \circ 1(t)=15$;

elseif mul (t 1$)==\operatorname{mu} 16(t)+u 16 \_1(t 1)$ $g \circ 1(t)=16$;

end

$\circ g \circ 2$

if $\operatorname{mu} 2(t 1)==\operatorname{mu} 1(t)+u 12(t 1)$ $g \circ 2(t)=1$

elseif mu2(t1) $==m u 2(t)+u 2 \_2(t 1)$ 


\section{APPENDIX G (Continued)}

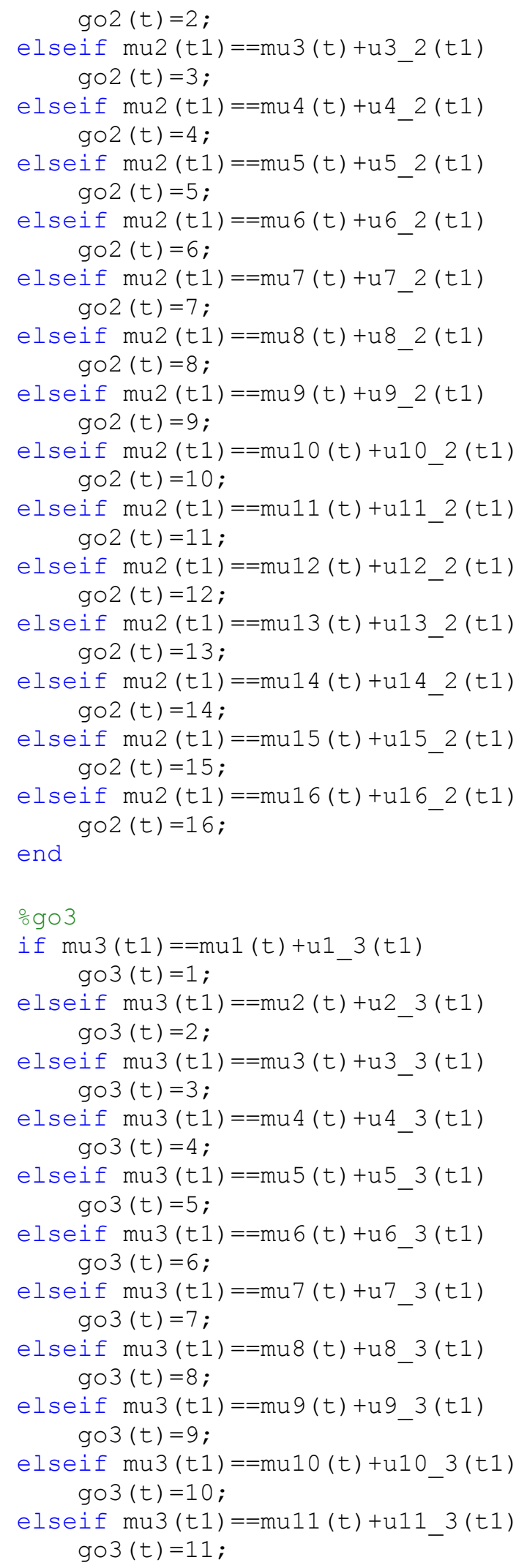




\section{APPENDIX G (Continued)}

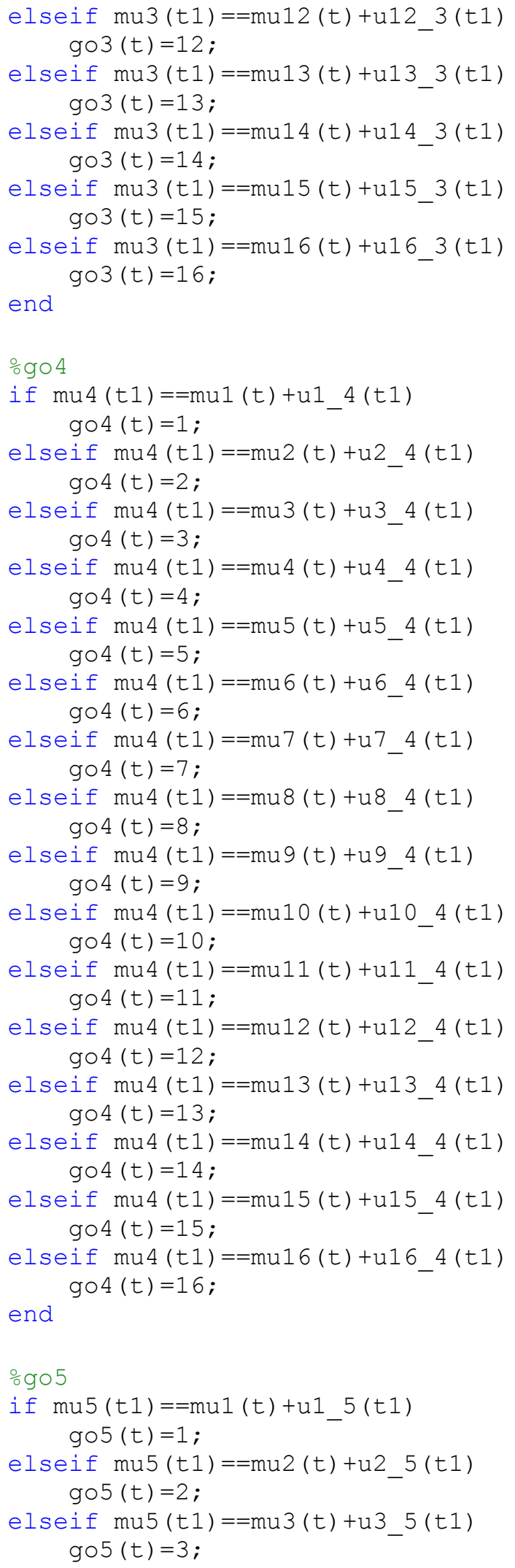




\section{APPENDIX G (Continued)}

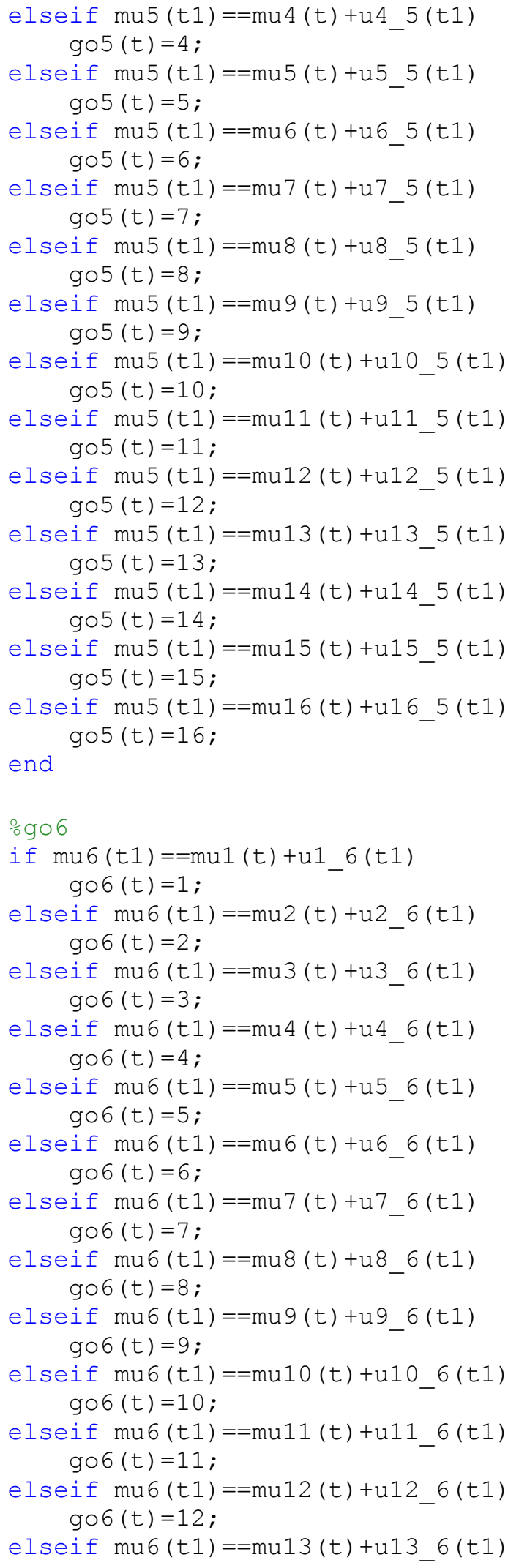




\section{APPENDIX G (Continued)}

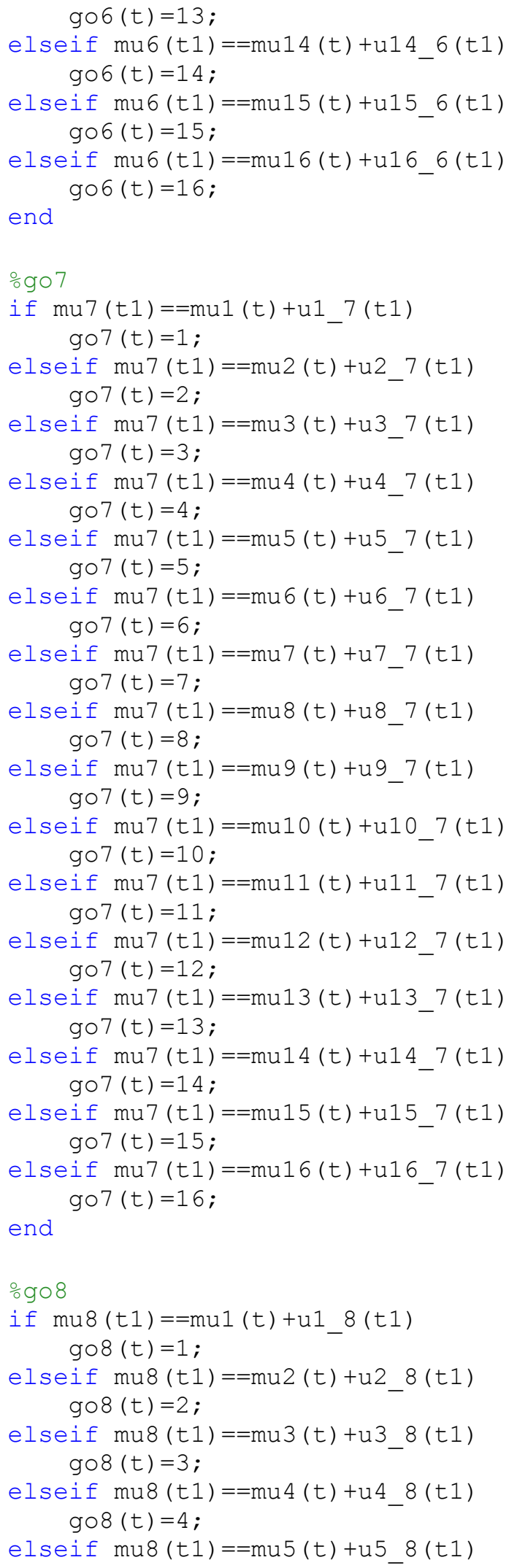




\section{APPENDIX G (Continued)}

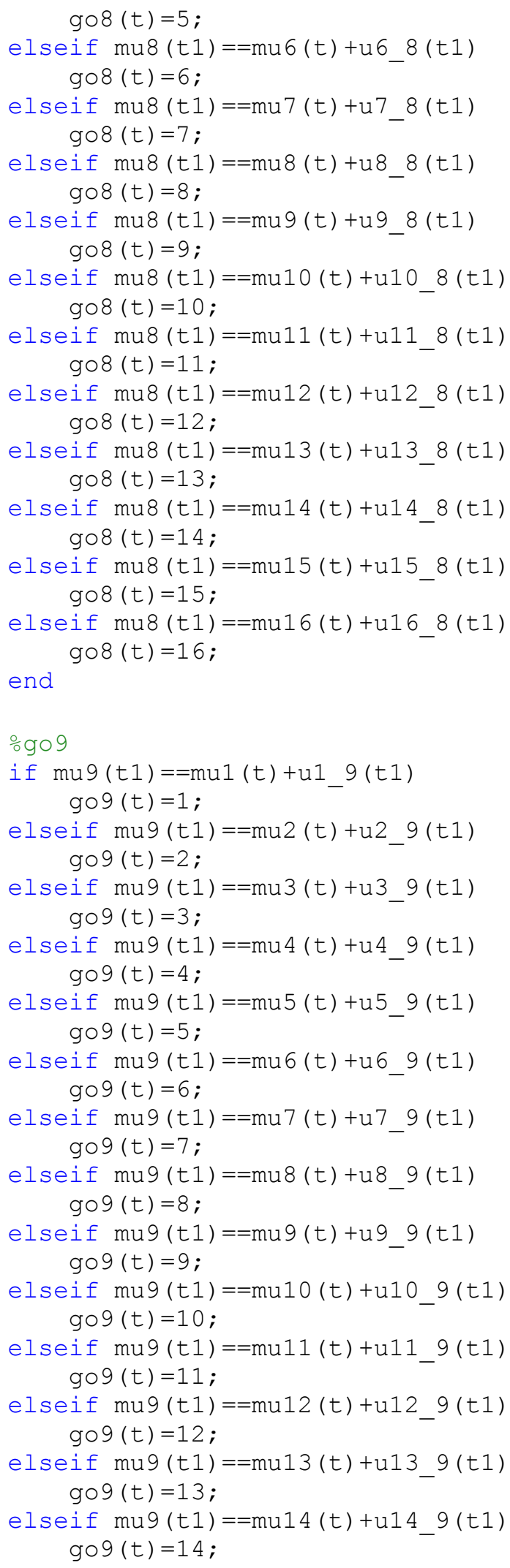




\section{APPENDIX G (Continued)}

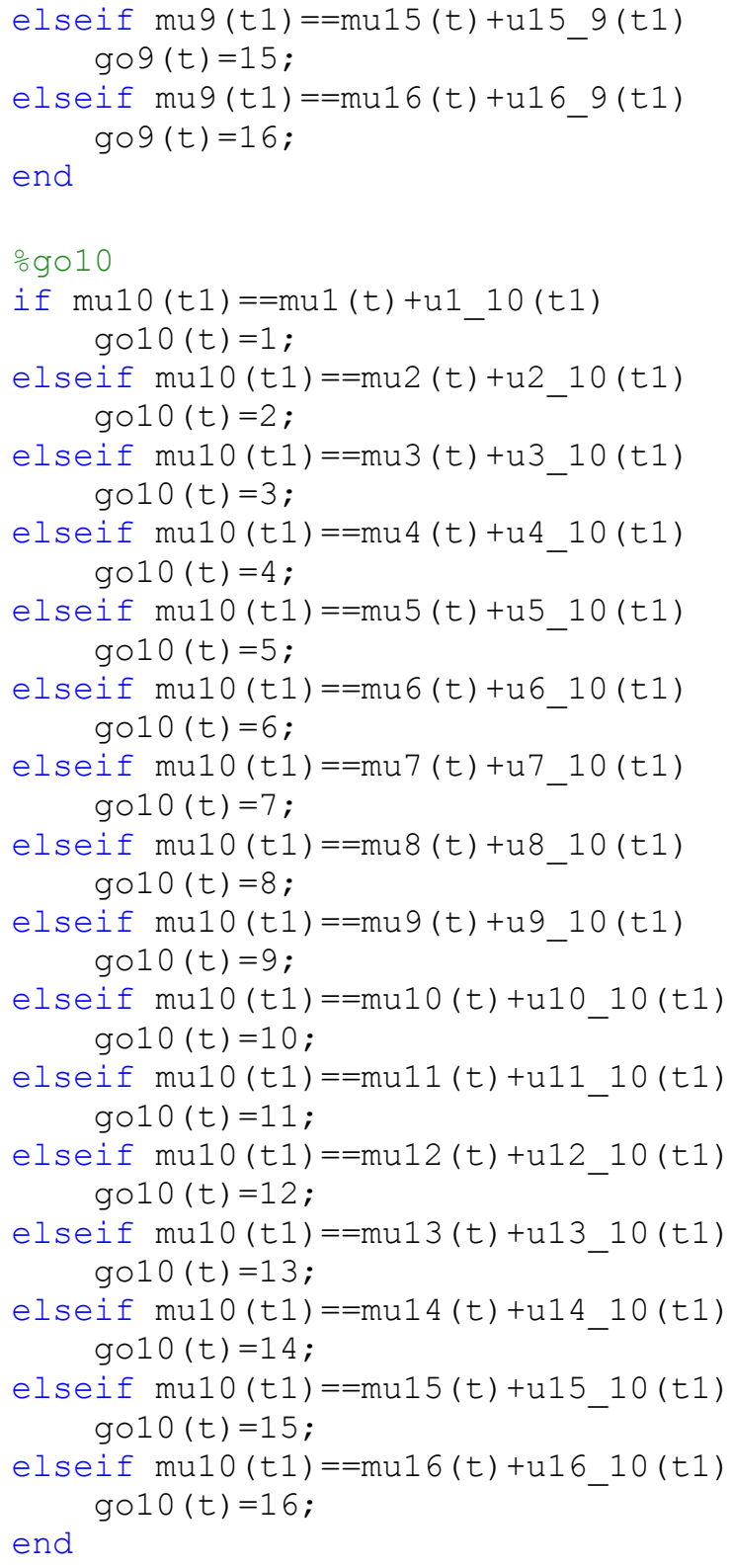




\section{APPENDIX G (Continued)}

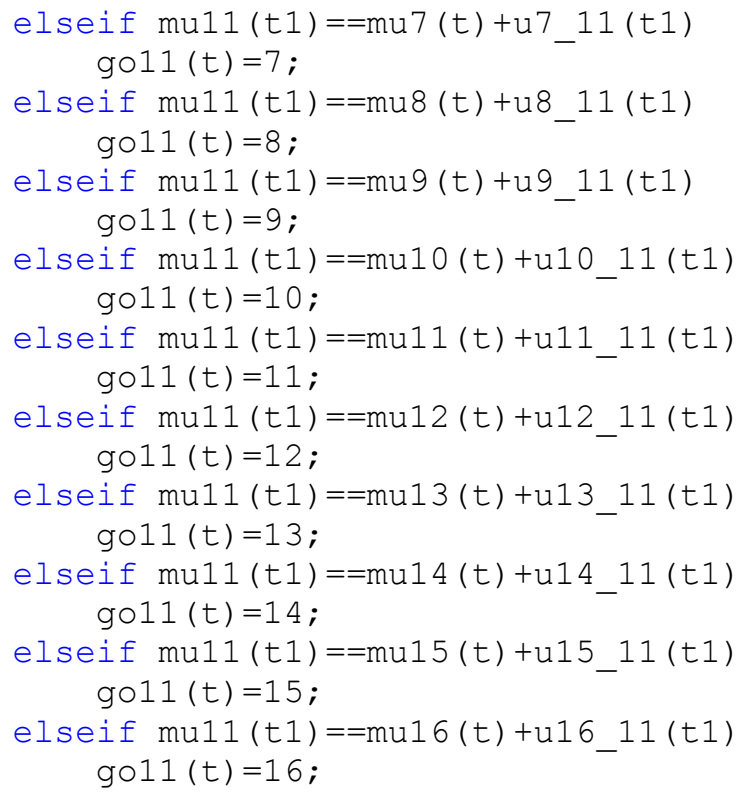




\section{APPENDIX G (Continued)}

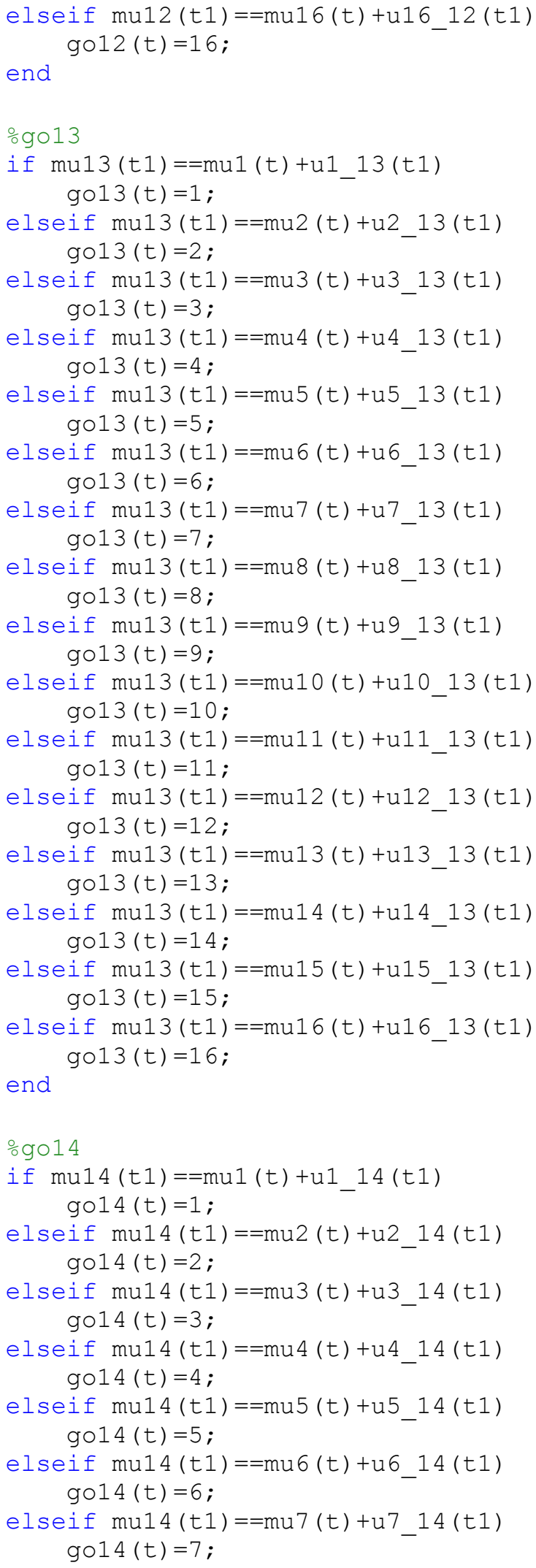




\section{APPENDIX G (Continued)}

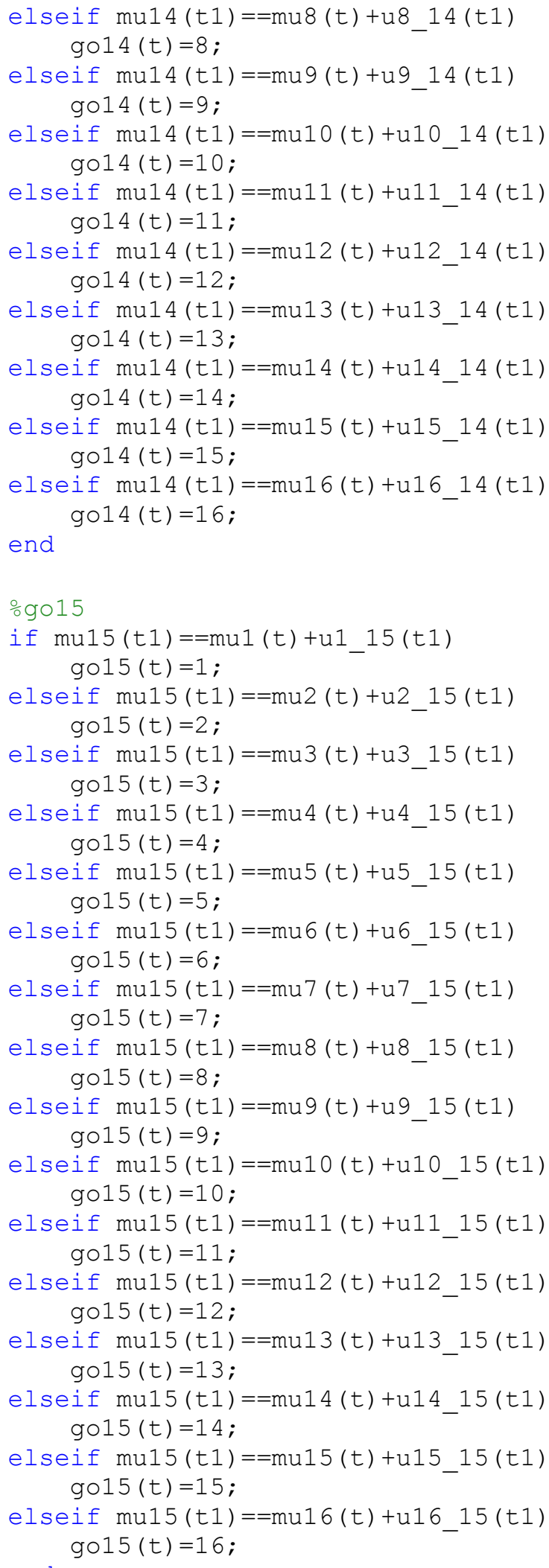




\section{APPENDIX G (Continued)}

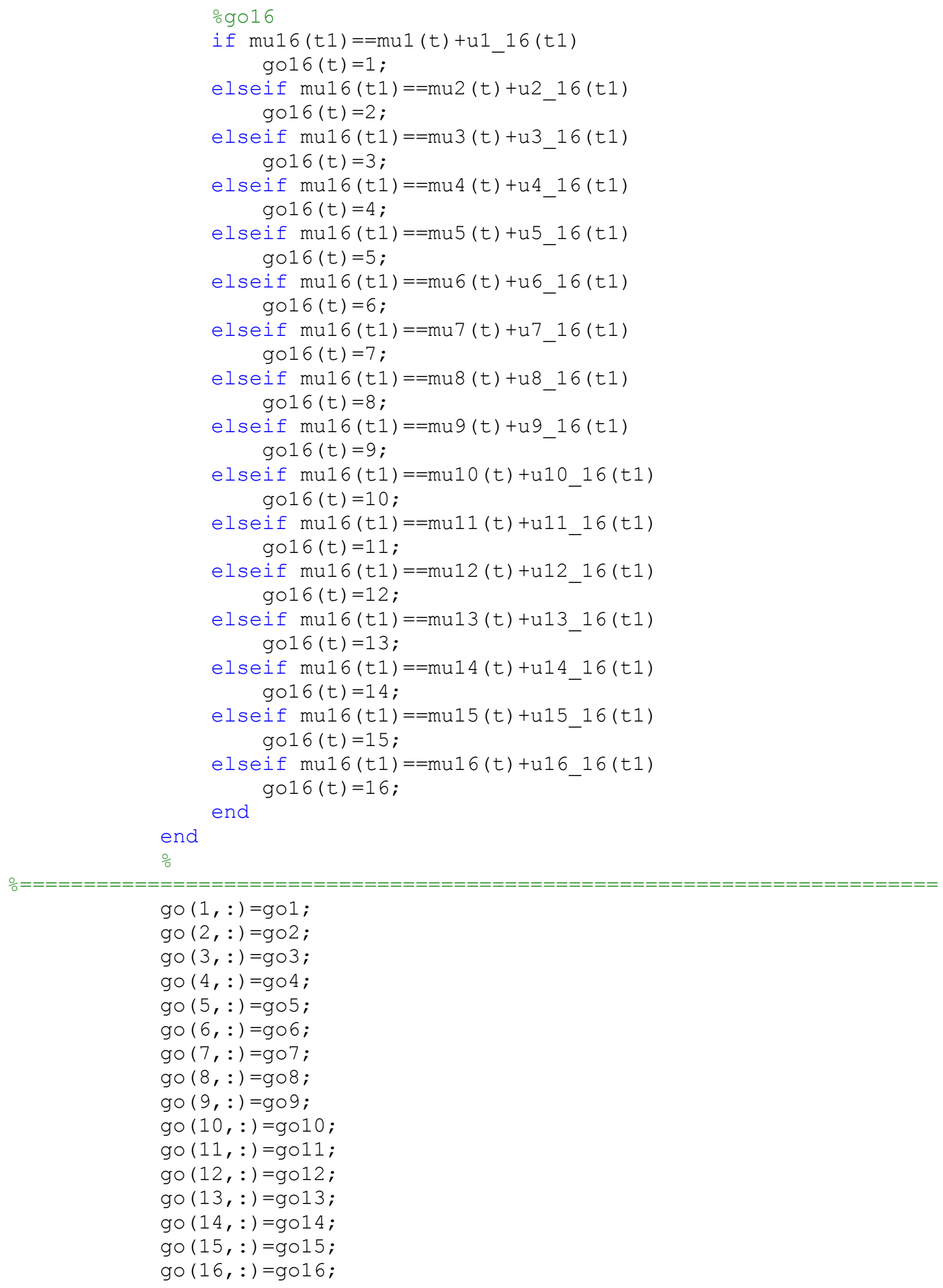




\section{APPENDIX G (Continued)}

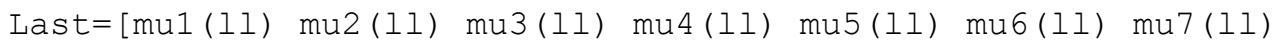
mu8(ll) mu9(ll) mulo(ll) mul1(ll) mul2(ll) mu13(ll) mu14(ll) mul5(ll) $\operatorname{mu} 16(11)]$;

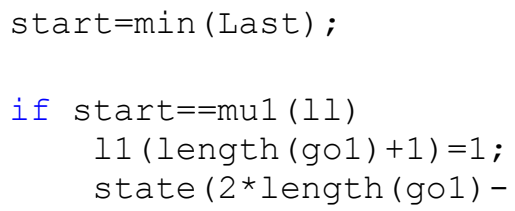

end 


\section{APPENDIX G (Continued)}

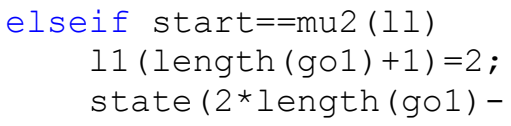

$1 / \operatorname{sqrt}(2)$;

$11($ length $(g \circ 1))=g \circ(3$, length $(g \circ 1))$;

for $t 2=$ length $(g \circ 1):-1: 2$

$11(t 2-1)=g \circ(11(t 2), t 2-1)$; 


\section{APPENDIX G (Continued)}

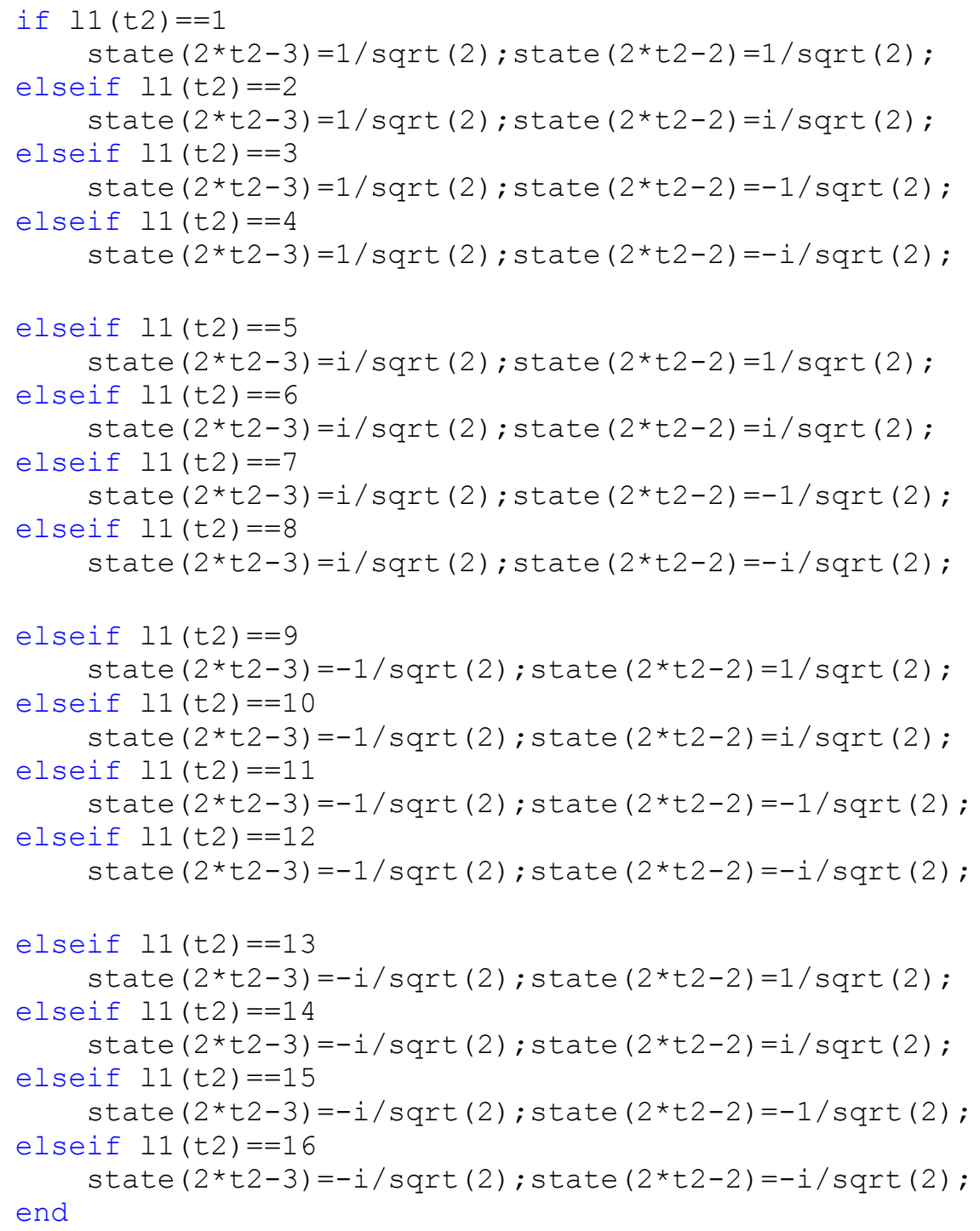




\section{APPENDIX G (Continued)}

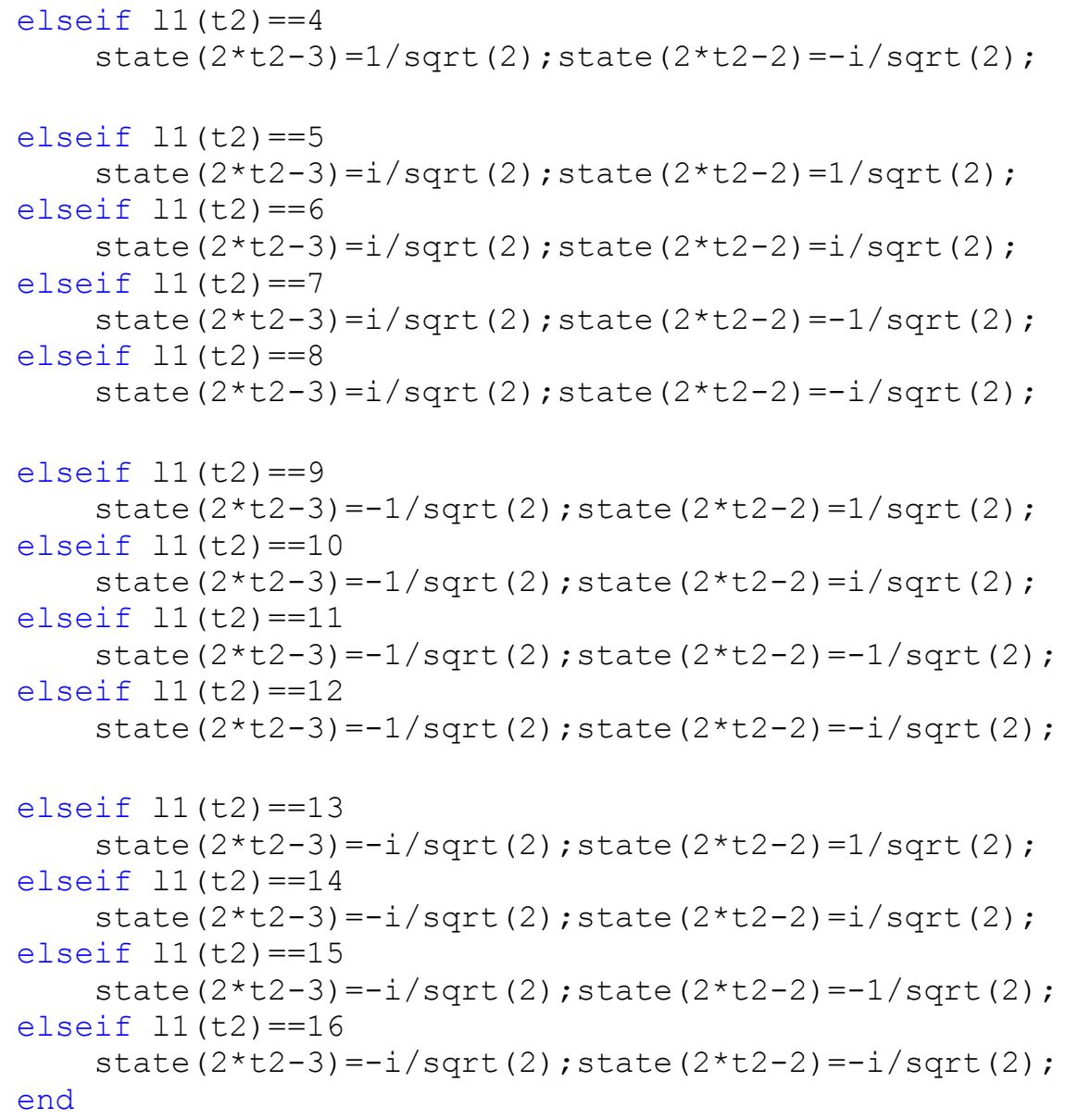




\section{APPENDIX G (Continued)}

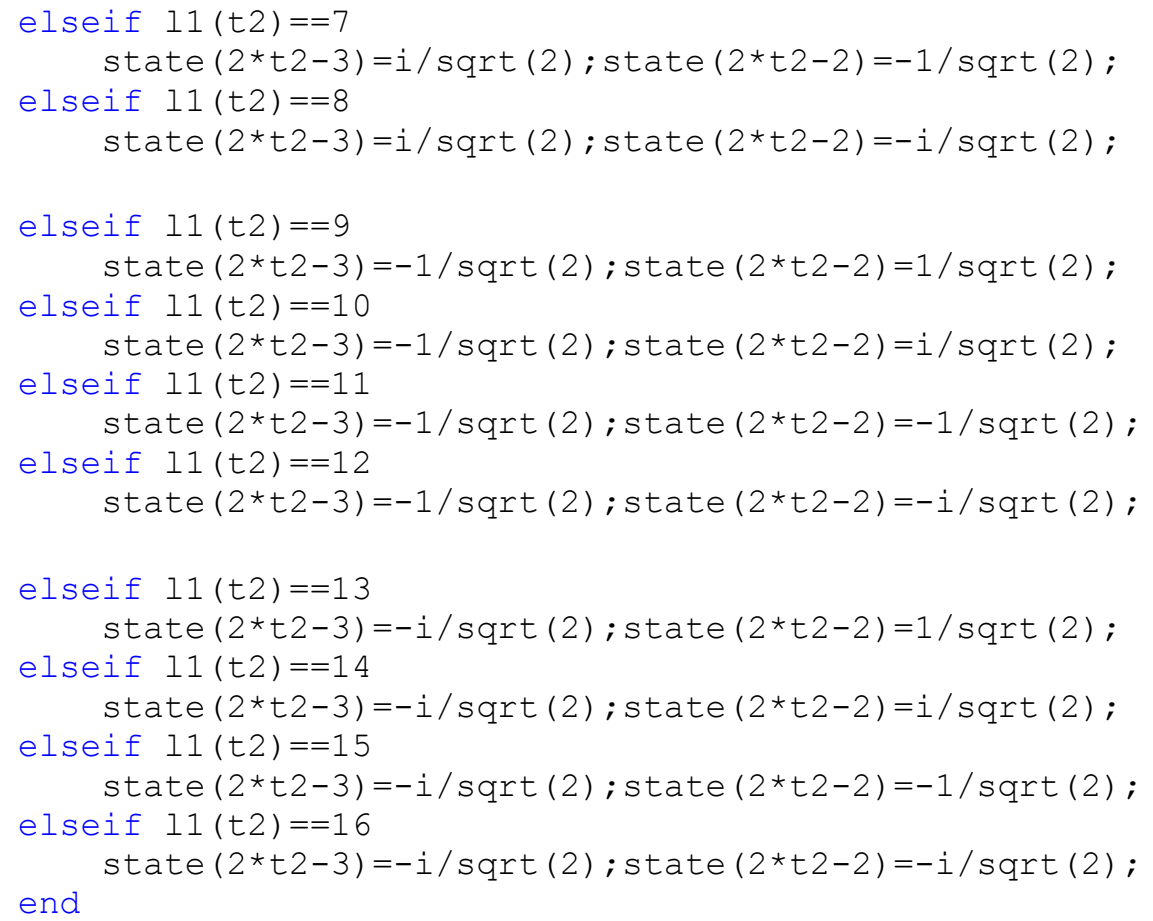




\section{APPENDIX G (Continued)}

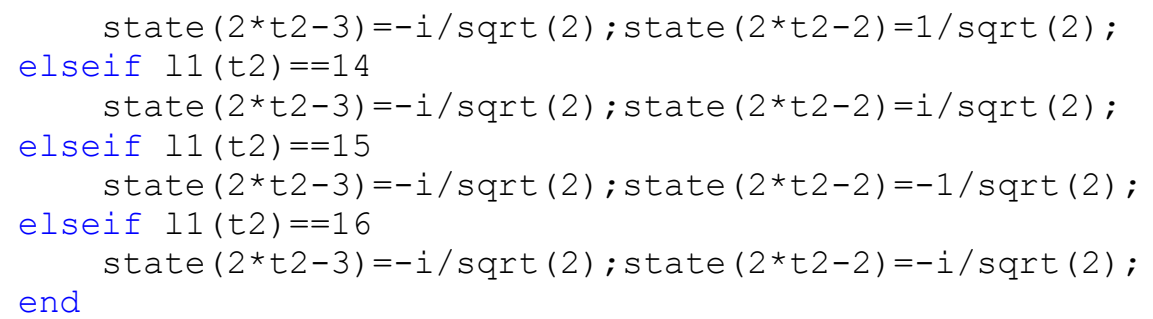




\section{APPENDIX G (Continued)}

end

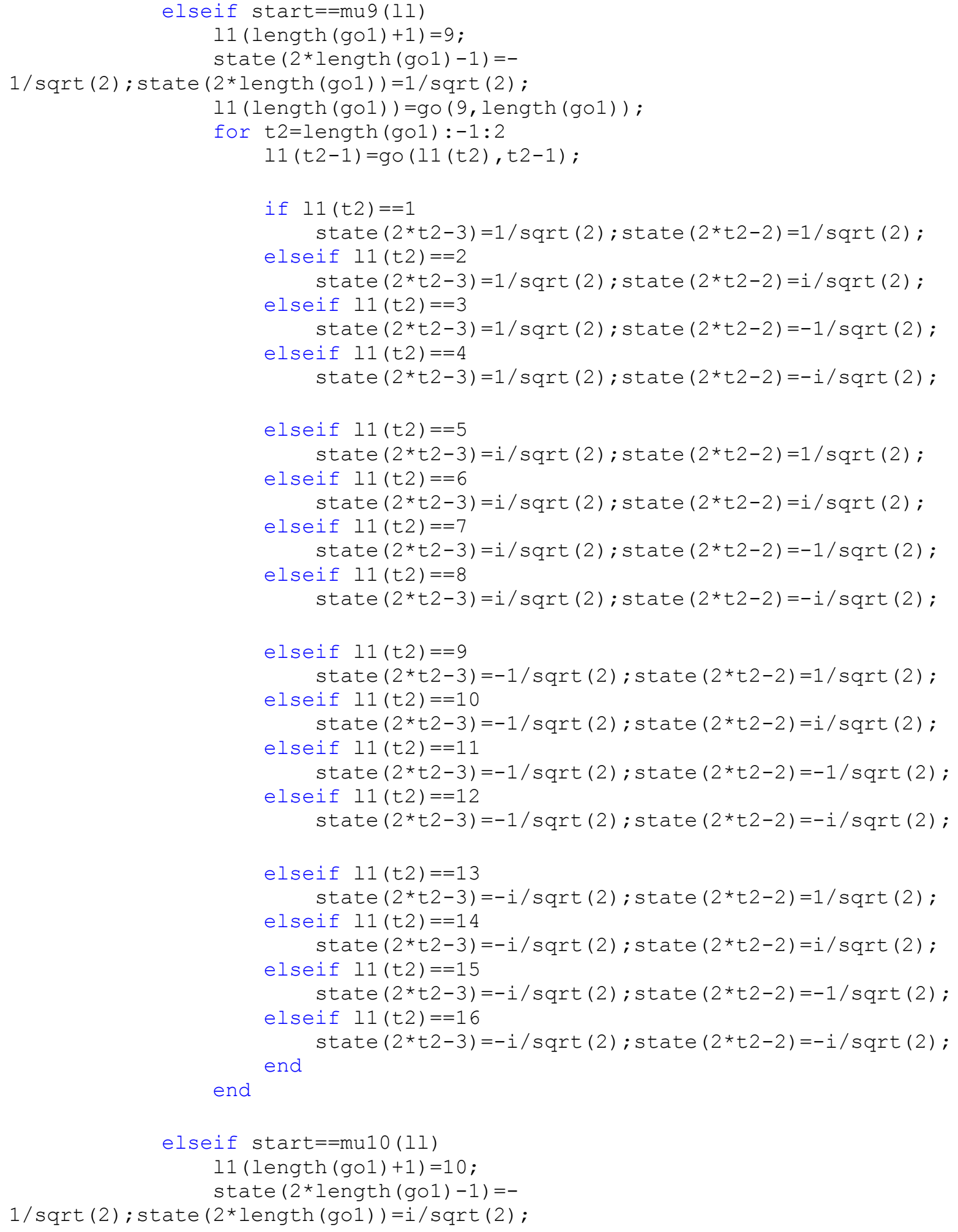




\section{APPENDIX G (Continued)}

$11($ length $(g \circ 1))=g \circ(10$, length $(g \circ 1))$;

for t2=length $(g \circ 1):-1: 2$

$11(t 2-1)=g \circ(11(t 2), t 2-1)$;

if $11(t 2)==1$ state $(2 * t 2-3)=1 / \operatorname{sqrt}(2) ; \operatorname{state}(2 * t 2-2)=1 / \operatorname{sqrt}(2)$; elseif $11(t 2)==2$ state $(2 * t 2-3)=1 / \operatorname{sqrt}(2) ; \operatorname{state}(2 * t 2-2)=i / \operatorname{sqrt}(2)$; elseif $11(t 2)==3$ state $(2 * t 2-3)=1 / \operatorname{sqrt}(2) ; \operatorname{state}(2 * t 2-2)=-1 / \operatorname{sqrt}(2)$; elseif $11(t 2)==4$ state $(2 * t 2-3)=1 / \operatorname{sqrt}(2) ;$ state $(2 * t 2-2)=-i / \operatorname{sqrt}(2)$;

elseif $11(t 2)==5$ state $(2 * t 2-3)=i / \operatorname{sqrt}(2) ; \operatorname{state}(2 * t 2-2)=1 / \operatorname{sqrt}(2)$; elseif $11(t 2)==6$ state $(2 * t 2-3)=i / \operatorname{sqrt}(2) ; \operatorname{state}(2 * t 2-2)=i / \operatorname{sqrt}(2)$; elseif $11($ t 2$)==7$ state $(2 * t 2-3)=i / \operatorname{sqrt}(2) ;$ state $(2 * t 2-2)=-1 / \operatorname{sqrt}(2)$; elseif $l 1(t 2)==8$ state $(2 * t 2-3)=i / \operatorname{sqrt}(2) ;$ state $(2 * t 2-2)=-i / \operatorname{sqrt}(2)$;

elseif $11(t 2)==9$ state $(2 \star t 2-3)=-1 / \operatorname{sqrt}(2) ;$ state $(2 \star t 2-2)=1 / \operatorname{sqrt}(2)$; elseif $11($ t 2$)==10$ state $(2 * t 2-3)=-1 / \operatorname{sqrt}(2) ; \operatorname{state}(2 * t 2-2)=i / \operatorname{sqrt}(2)$; elseif $11($ t 2$)==11$ state $(2 * t 2-3)=-1 / \operatorname{sqrt}(2) ; \operatorname{state}(2 * t 2-2)=-1 / \operatorname{sqrt}(2)$; elseif $l 1($ t 2$)==12$ state $(2 * t 2-3)=-1 / \operatorname{sqrt}(2) ; \operatorname{state}(2 * t 2-2)=-i / \operatorname{sqrt}(2) ;$

elseif $11($ t 2$)==13$ state $(2 * t 2-3)=-i / \operatorname{sqrt}(2) ; \operatorname{state}(2 * t 2-2)=1 / \operatorname{sqrt}(2)$; elseif $11($ t 2$)==14$ state $(2 * t 2-3)=-i / \operatorname{sqrt}(2) ; \operatorname{state}(2 * t 2-2)=i / \operatorname{sqrt}(2)$; elseif $l 1($ t 2$)==15$ state $(2 \star t 2-3)=-i / \operatorname{sqrt}(2) ; \operatorname{state}(2 \star t 2-2)=-1 / \operatorname{sqrt}(2)$; elseif $11($ t 2$)==16$ state $(2 * t 2-3)=-i / \operatorname{sqrt}(2) ; \operatorname{state}(2 * t 2-2)=-i / \operatorname{sqrt}(2)$;

end

elseif start $==\operatorname{mul}(11)$

$11($ length $(g \circ 1)+1)=11$;

1/sqrt (2) ; state $(2 *$ length $(g \circ 1)-1)=-1 / \operatorname{sqrt}(2)$; $\operatorname{state}(2 *$ length $(g \circ 1))=-$

$11($ length $(g \circ 1))=g \circ(11$, length $(g \circ 1))$;

for $t 2=$ length $(g \circ 1):-1: 2$

$11(t 2-1)=g \circ(11(t 2), t 2-1)$;

if $11(t 2)==1$ state $(2 * t 2-3)=1 / \operatorname{sqrt}(2) ; \operatorname{state}(2 * t 2-2)=1 / \operatorname{sqrt}(2)$; elseif $11(t 2)==2$ 


\section{APPENDIX G (Continued)}

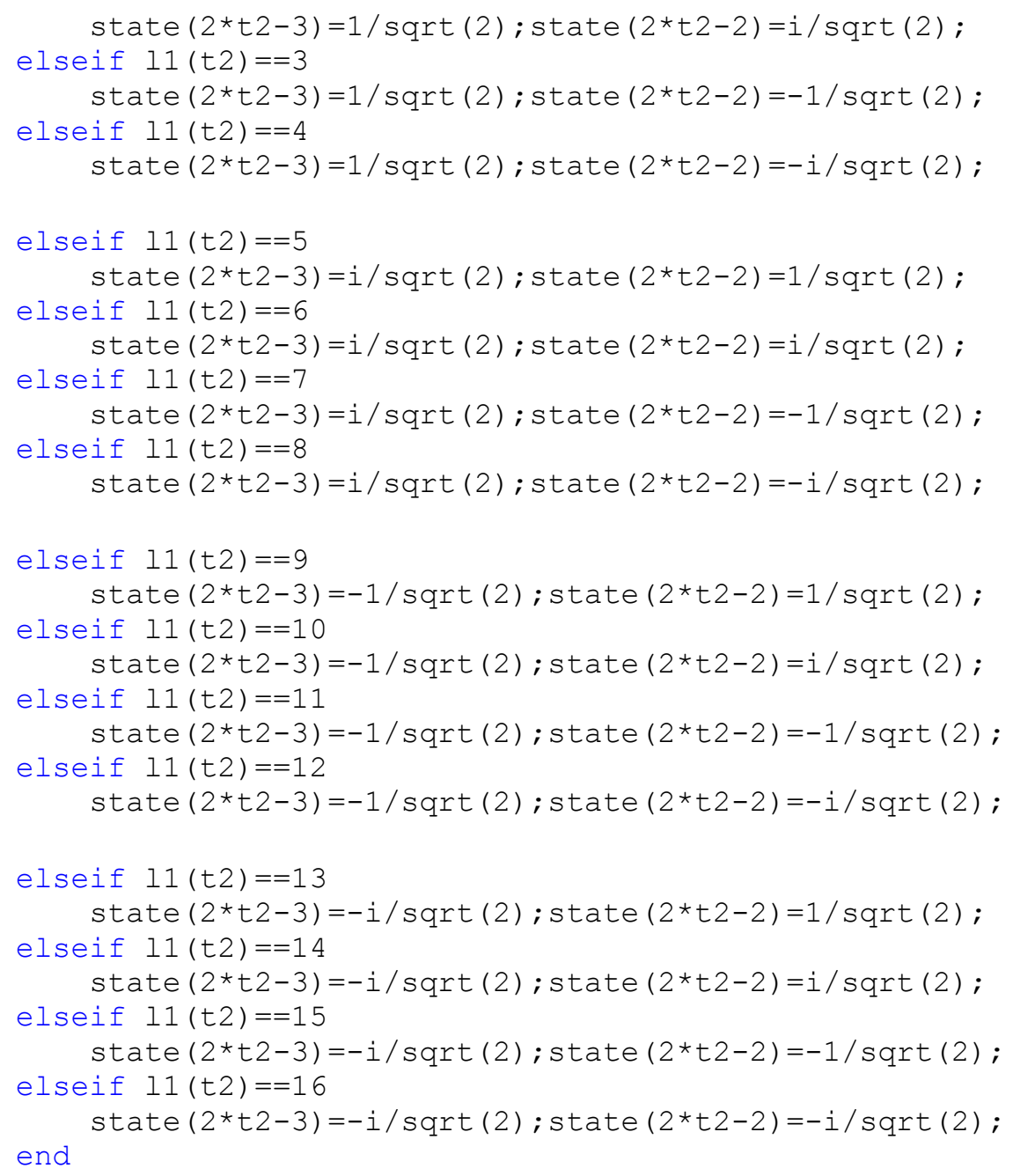




\section{APPENDIX G (Continued)}

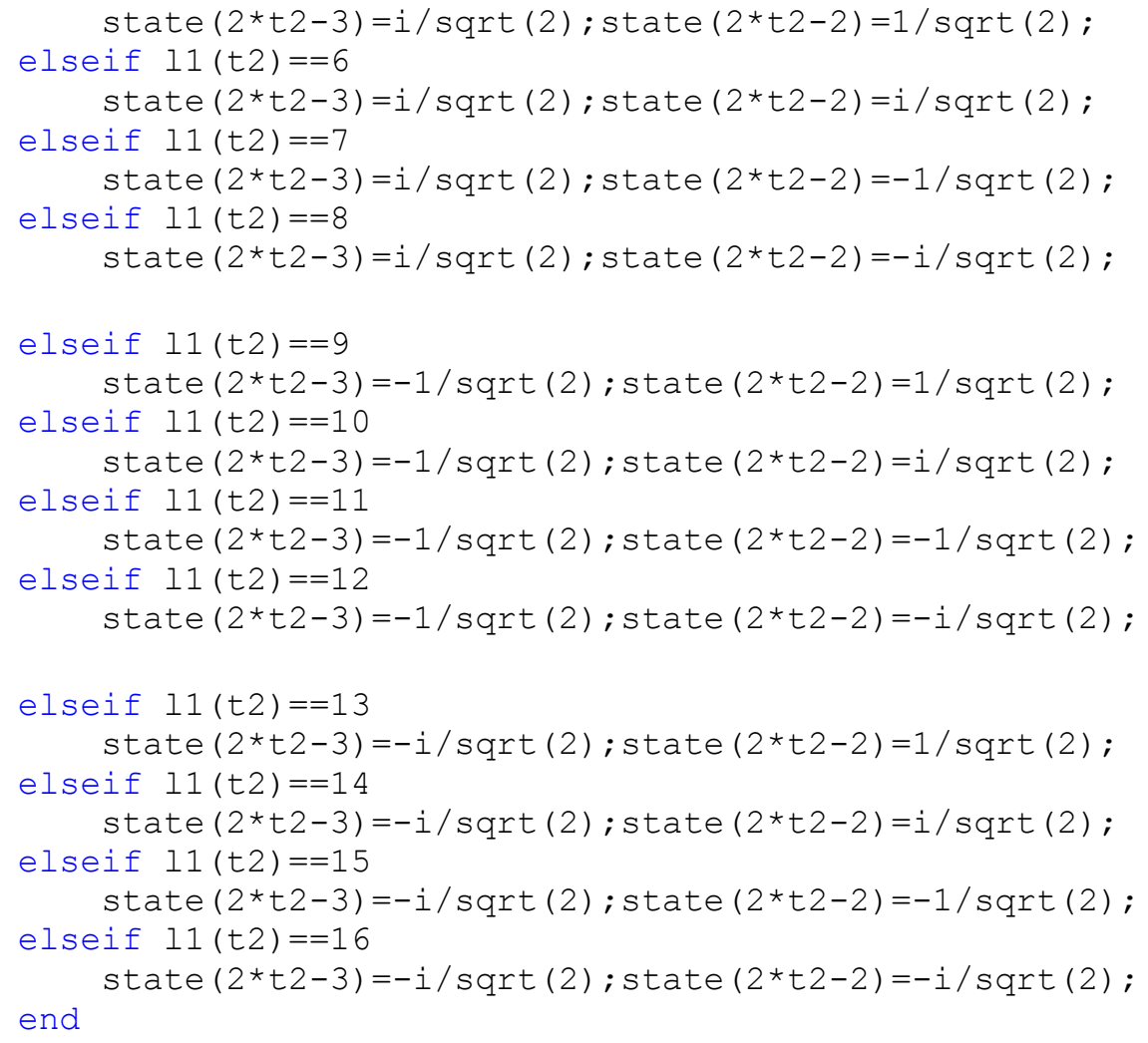




\title{
APPENDIX G (Continued)
}

\author{
elseif $11($ t 2$)==9$ \\ state $(2 * t 2-3)=-1 / \operatorname{sqrt}(2) ; \operatorname{state}(2 * t 2-2)=1 / \operatorname{sqrt}(2)$; \\ elseif $11(t 2)==10$ \\ state $(2 * t 2-3)=-1 / \operatorname{sqrt}(2) ; \operatorname{state}(2 * t 2-2)=i / \operatorname{sqrt}(2)$; \\ elseif $11($ t 2$)==11$ \\ state $(2 * t 2-3)=-1 / \operatorname{sqrt}(2) ; \operatorname{state}(2 * t 2-2)=-1 / \operatorname{sqrt}(2)$; \\ elseif $11(t 2)==12$




\title{
APPENDIX G (Continued)
}

\author{
elseif $11(t 2)==12$ \\ state $(2 * t 2-3)=-1 / \operatorname{sqrt}(2) ; \operatorname{state}(2 * t 2-2)=-i / \operatorname{sqrt}(2)$; \\ elseif $11(t 2)==13$ \\ state $(2 * t 2-3)=-i / \operatorname{sqrt}(2) ; \operatorname{state}(2 * t 2-2)=1 / \operatorname{sqrt}(2)$; \\ elseif $11($ t 2$)==14$ \\ state $(2 * t 2-3)=-i / \operatorname{sqrt}(2) ; \operatorname{state}(2 * t 2-2)=i / \operatorname{sqrt}(2)$; \\ elseif $11(t 2)==15$ \\ state $(2 * t 2-3)=-i / \operatorname{sqrt}(2) ; \operatorname{state}(2 * t 2-2)=-1 / \operatorname{sqrt}(2)$; \\ elseif $l 1(t 2)==16$ \\ state $(2 * t 2-3)=-i / \operatorname{sqrt}(2) ; \operatorname{state}(2 * t 2-2)=-i / \operatorname{sqrt}(2)$; \\ end \\ end \\ elseif start $==\operatorname{mu} 15(11)$ \\ $11($ length $(g \circ 1)+1)=15$; \\ $1 / \operatorname{sqrt}(2)$ \\ state $(2 *$ length $(g \circ 1)-1)=-i / \operatorname{sqrt}(2)$; state $(2 *$ length $(g \circ 1))=-$ \\ $11($ length $(g \circ 1))=g \circ(15$, length $(g \circ 1))$; \\ for $t 2=$ length $(g \circ 1):-1: 2$ \\ $11(t 2-1)=g \circ(11(t 2), t 2-1)$; \\ if $11($ t 2$)==1$ \\ state $(2 * t 2-3)=1 / \operatorname{sqrt}(2) ; \operatorname{state}(2 * t 2-2)=1 / \operatorname{sqrt}(2)$; \\ elseif $11(t 2)==2$ \\ state $(2 * t 2-3)=1 / \operatorname{sqrt}(2) ; \operatorname{state}(2 * t 2-2)=i / \operatorname{sqrt}(2)$; \\ elseif $11(t 2)==3$ \\ state $(2 * t 2-3)=1 / \operatorname{sqrt}(2)$; state $(2 * t 2-2)=-1 / \operatorname{sqrt}(2)$; \\ elseif $l 1(t 2)==4$ \\ state $(2 * t 2-3)=1 / \operatorname{sqrt}(2) ;$ state $(2 * t 2-2)=-i / \operatorname{sqrt}(2)$; \\ elseif $11(t 2)==5$ \\ state $(2 * t 2-3)=i / \operatorname{sqrt}(2) ; \operatorname{state}(2 * t 2-2)=1 / \operatorname{sqrt}(2)$; \\ elseif $11($ t 2$)==6$ \\ state $(2 * t 2-3)=i / \operatorname{sqrt}(2) ;$ state $(2 * t 2-2)=i / \operatorname{sqrt}(2)$; \\ elseif $11(t 2)==7$ \\ state $(2 * t 2-3)=i / \operatorname{sqrt}(2) ;$ state $(2 * t 2-2)=-1 / \operatorname{sqrt}(2)$; \\ elseif $11(t 2)==8$ \\ state $(2 * t 2-3)=i / \operatorname{sqrt}(2) ; \operatorname{state}(2 * t 2-2)=-i / \operatorname{sqrt}(2)$; \\ elseif $11(t 2)==9$ \\ state $(2 * t 2-3)=-1 / \operatorname{sqrt}(2) ; \operatorname{state}(2 * t 2-2)=1 / \operatorname{sqrt}(2)$; \\ elseif $11($ t 2$)==10$ \\ state $(2 * t 2-3)=-1 / \operatorname{sqrt}(2) ; \operatorname{state}(2 * t 2-2)=i / \operatorname{sqrt}(2)$; \\ elseif $11(t 2)==11$ \\ state $(2 * t 2-3)=-1 / \operatorname{sqrt}(2) ; \operatorname{state}(2 * t 2-2)=-1 / \operatorname{sqrt}(2)$; \\ elseif $11(t 2)==12$ \\ state $(2 * t 2-3)=-1 / \operatorname{sqrt}(2) ; \operatorname{state}(2 * t 2-2)=-i / \operatorname{sqrt}(2)$; \\ elseif $11(t 2)==13$ \\ state $(2 * t 2-3)=-i / \operatorname{sqrt}(2) ; \operatorname{state}(2 * t 2-2)=1 / \operatorname{sqrt}(2)$; \\ elseif $11(t 2)==14$ \\ state $(2 * t 2-3)=-i / \operatorname{sqrt}(2) ; \operatorname{state}(2 * t 2-2)=i / \operatorname{sqrt}(2)$;
}




\section{APPENDIX G (Continued)}

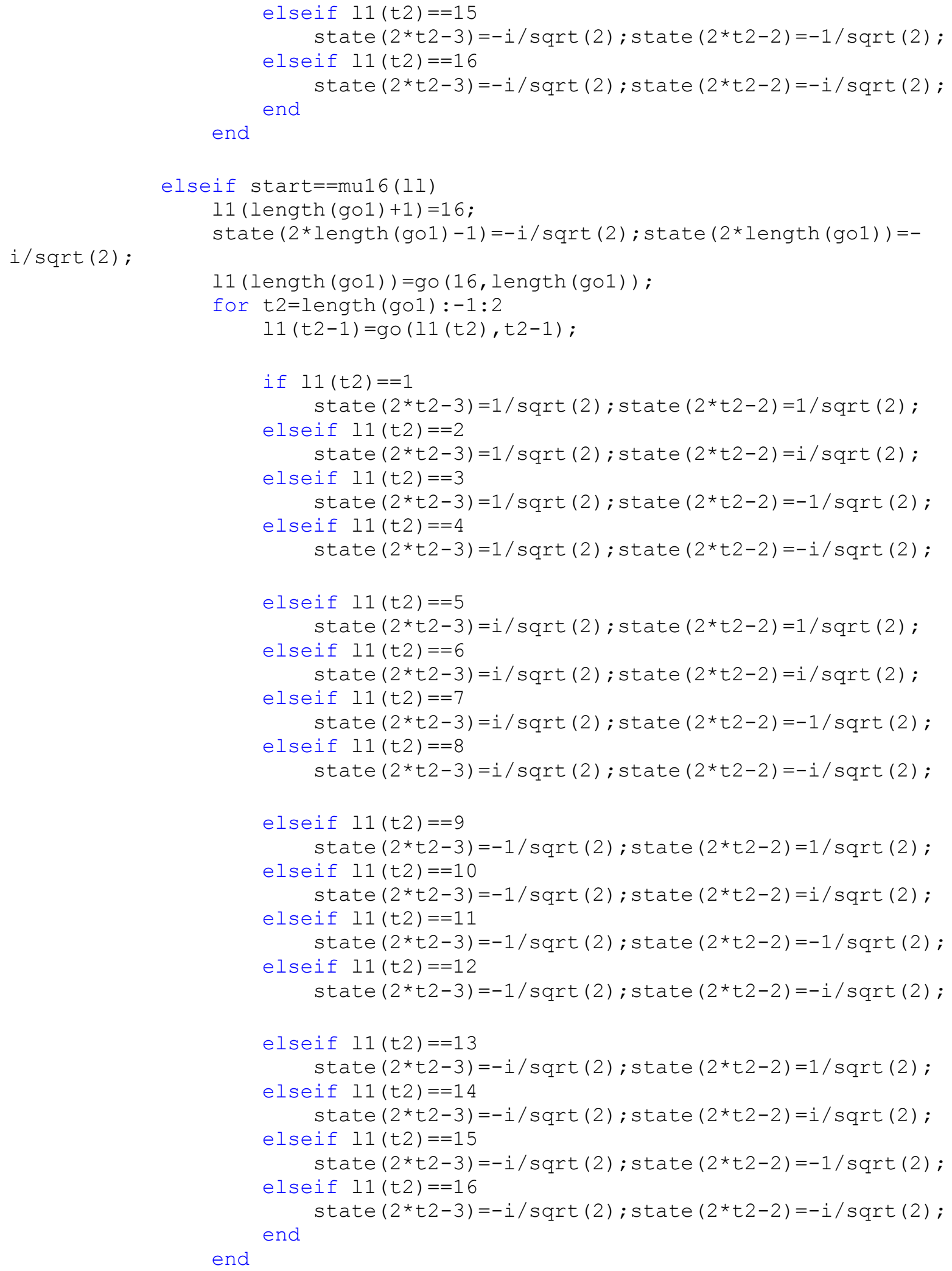




\section{APPENDIX G (Continued)}

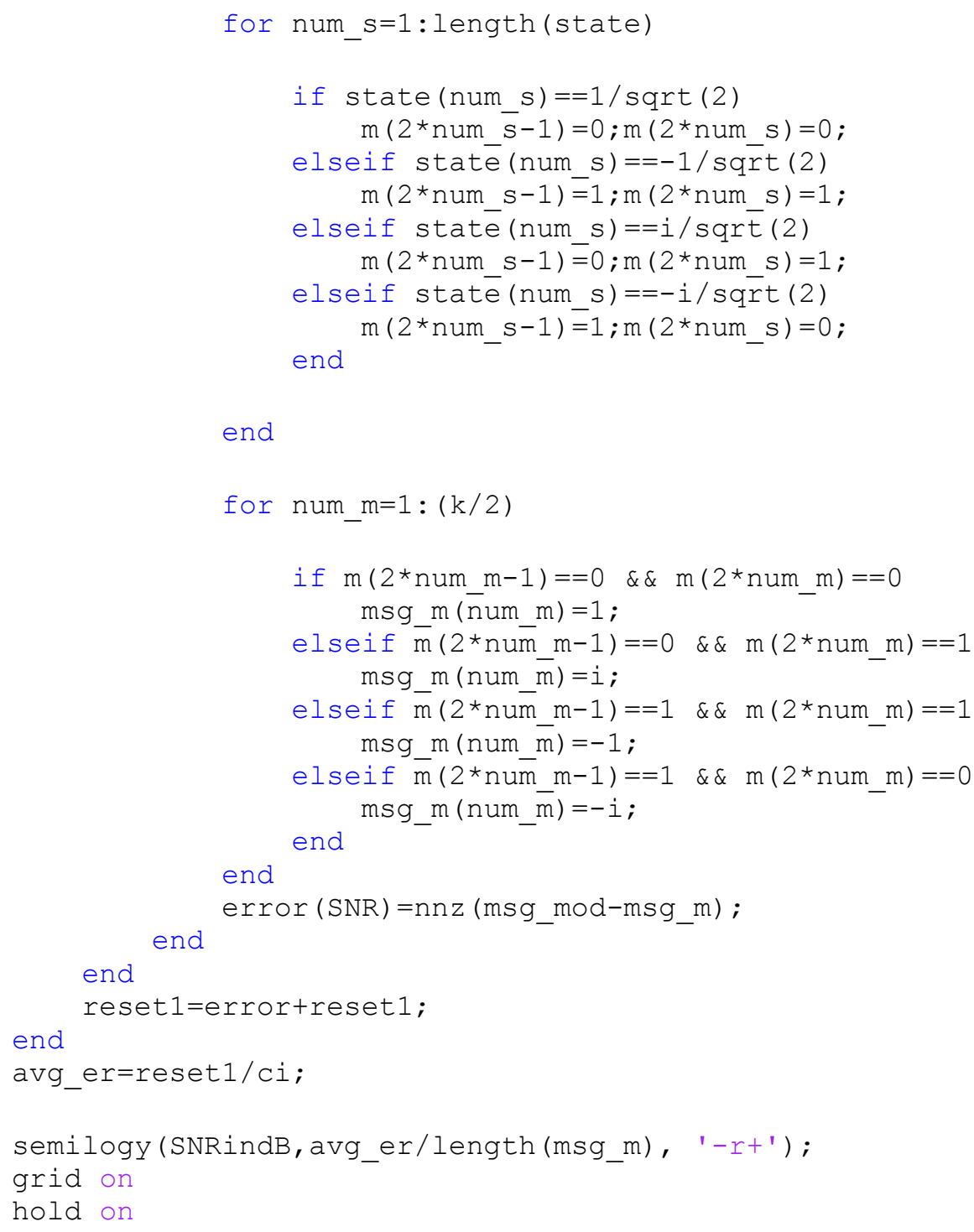


